\title{
Homomorphisms from AH-algebras
}

\author{
Huaxin Lin
}

\begin{abstract}
Let $C$ be a general unital AH-algebra and let $A$ be a unital simple $C^{*}$-algebra with tracial rank at most one. Suppose that $\varphi, \psi: C \rightarrow A$ are two unital monomorphisms. We show that $\varphi$ and $\psi$ are approximately unitarily equivalent if and only if

$$
\begin{aligned}
{[\varphi] } & =[\psi] \text { in } K L(C, A), \\
\varphi_{\sharp} & =\psi_{\sharp} \text { and } \\
\varphi_{\rho} & =\psi_{\rho},
\end{aligned}
$$

where $\varphi_{\sharp}$ and $\psi_{\sharp}$ are continuous affine maps from tracial state space $T(A)$ of $A$ to faithful tracial state space $T_{\mathrm{f}}(C)$ of $C$ induced by $\varphi$ and $\psi$, respectively, and $\varphi_{\rho}$ and $\psi_{\rho}$ are induced homomorphisms from $K_{1}(C)$ into $\operatorname{Aff}(T(A)) / \overline{\rho_{A}\left(K_{0}(A)\right)}$, where $\operatorname{Aff}(T(A))$ is the space of all real affine continuous functions on $T(A)$ and $\rho_{A}\left(K_{0}(A)\right)$ is the closure of the image of $K_{0}(A)$ in the affine space $\operatorname{Aff}(T(A))$. In particular, the above holds for $C=C(X)$, the algebra of continuous functions on a compact metric space. An approximate version of this is also obtained. We also show that, given a triple of compatible elements $\kappa \in K L_{e}(C, A)^{++}$, an affine map $\gamma: T(C) \rightarrow T_{\mathrm{f}}(C)$ and a homomorphism $\alpha: K_{1}(C) \rightarrow \operatorname{Aff}(T(A)) / \overline{\rho_{A}\left(K_{0}(A)\right)}$, there exists a unital monomorphism $\varphi: C \rightarrow A$ such that $[h]=\kappa, h_{\sharp}=\gamma$ and $\varphi_{\rho}=\alpha$.
\end{abstract}

\section{Introduction}

Let $X$ be a compact metric space and let $A$ be a unital simple $C^{*}$-algebra. Let $\varphi, \psi: C(X) \rightarrow A$ be two homomorphisms. We study the problem when these two maps from $C(X)$, the commutative $C^{*}$-algebra of continuous functions on $X$, into $A$ are approximately unitarily equivalent, i.e., when there exists a sequence of unitaries $\left\{u_{n}\right\} \subset A$ such that

$$
\lim _{n \rightarrow \infty} u_{n}^{*} \psi(f) u_{n}=\varphi(f) \text { for all } f \in C(X) .
$$

In the case that $X$ is a compact subset of the plane and $A$ is the $n \times n$ matrix algebra, two such maps are unitarily equivalent if and only if the corresponding normal matrices have the same set of eigenvalues (counting multiplicity). Brown-Douglass-Fillmore's study of essentially normal operators led to the following theorem: Two unital monomorphisms from $C(X)$ (when $X$ is a compact subset of the plane) into the Calkin algebra are unitarily equivalent if and only if they induce the same homomorphism from $K_{1}(C(X))$ into $\mathbb{Z}$. It should be noted that both the $n \times n$ matrix algebra and the Calkin algebra are unital simple $C^{*}$-algebras of real rank zero.

Unital separable commutative $C^{*}$-algebras are of the form $C(X)$ for some compact metric space by the Gelfand transformation. Therefore the study of $C^{*}$-algebras may be viewed as the study of non-commutative topology. As in the topology, one studies continuous maps between spaces, in $C^{*}$-algebra theory, one studies the homomorphisms from one $C^{*}$-algebra to another. In this point of view, the study of homomorphisms from one $C^{*}$-algebra to another is one of the fundamental problems in the $C^{*}$-algebra theory. At the present paper, we assume that the target algebra is a unital simple $C^{*}$-algebra, which conforms to the previous two mentioned cases. Simple $C^{*}$-algebras may also be viewed as the opposite end of commutative $C^{*}$-algebras. 
For the source algebra, we begin with the case that it is the commutative $C^{*}$-algebra following the two above mentioned cases. However, we will study the case that source algebras are general unital AH-algebras (They are not necessarily simple, nor of slow dimension growth).

Let $\varphi, \psi: C(X) \rightarrow A$ be two unital homomorphisms and let $I=\operatorname{ker} \varphi$. Then $I=\operatorname{ker} \psi$, if $\varphi$ and $\psi$ are approximately unitarily equivalent. Therefore, one may study the induced homomorphisms from $C(X) / I$ instead. Note that $C(X) / I$ is isomorphic to $C(Y)$ for some compact subset of $X$. To simplify the matter, we will only study monomorphisms. The problem has been studied (for some earlier results, for example, see [8] and [9] ). Dadarlat ([2]) showed that, if $C=C(X)$ and $A$ is a unital purely infinite simple $C^{*}$-algebra (such as the Calkin algebra), then two unital monomorphisms from $C$ into $A$ are approximately unitarily equivalent if and only if they induce the same element in $K L(C, A)$. When the target $C^{*}$-algebras are finite, other invariants such as traces have to be considered. When $A$ is a unital simple $C^{*}$-algebra with stable rank one, real rank zero, weakly unperforated $K_{0}(A)$ and a unique tracial state, it is shown in [6] that $\varphi$ and $\psi$ are approximately unitarily equivalent if and only if $[\varphi]=[\psi]$ in $K L(C(X), A)$ and $\tau \circ \varphi=\tau \circ \psi$. When the real rank of $A$ is not zero one needs additional data to determine when $\varphi$ and $\psi$ are approximately unitarily equivalent. In fact, it is shown ([21]) that when $C$ is a some special unital AH-algebra and $A$ is a unital simple $C^{*}$-algebra with tracial rank at most one, two unital monomorphisms $\varphi, \psi: C \rightarrow A$ are approximately unitarily equivalent if and only $[\varphi]=[\psi], \varphi_{\sharp}=\psi_{\sharp}$ and $\varphi^{\ddagger}=\psi^{\ddagger}$, where $\varphi_{\sharp}$ and $\varphi^{\ddagger}$ will be defined below ((2.1) and (2.4)). The technical condition imposed on $C(X)$ is basically said that, $K$-theoretically speaking, $C(X)$ has a lower rank. In this paper this restriction on $A H$-algebras has been removed. A complete criterion is given for two unital monomorphisms from a general $A H$-algebra into a unital simple $C^{*}$-algebra with tracial rank at most one being approximately unitarily equivalent.

One of the long standing problems in the classification theory is to classify locally AHalgebra with no dimension growth. The problem could be solved if one could establish a version of Gong's decomposition theorem which allows maps that are not exactly homomorphisms. Over more than a decade, since the proof of Gong's decomposition theorem first appeared, the technical difficulty to generalize it to include almost multiplicative maps had remained elusive. This author's many attempts failed during these years. It is the desire to prove that unital simple locally AH-algebras with no dimension growth can also be classified by their Elliott invariant drew author's attention again to Gong's decomposition theorem. One application of the results in this paper will be the proof that unital simple locally AH-algebras with slow dimension growth are classifiable by the Elliott invariant ([22]).

We also believe that the main results presented here have their own independent interest as discussed at the beginning of this introduction.

The paper is organized as follows: Section 2 serves largely as preliminaries for the whole paper. In Section 3, we prove Theorem 3.6 which is the main technical advance of this paper. In Section 4, we collect a number of miscellaneous lemmas which will be used in the proof of the main results. In Section 5, we prove the main results. To complete our results and make application possible, in Section 6, we provide the description of the range of approximate unitary equivalence classes of unital monomorphisms from a unital AH-algebra to a unital simple $C^{*}$ algebra of tracial rank at most one. Applications to the study of tracial rank and classification of unital simple locally AH-algebras will appear elsewhere ([22]).

Acknowledgment Most of this work was done when the author was in East China Normal University in the summer 2010. This work is partially supported by University of Oregon, East China Normal University and a NSF grant. The author would like to thank Claude Schochet who pointed to us there was an awkward computation in an earlier version of this paper which was now avoided. 


\section{Preliminaries}

2.1. Let $A$ be a unital $C^{*}$-algebra. Denote by $T(A)$ the convex set of tracial states of $C$. Denote by $T_{\mathrm{f}}(A)$ the convex set of all faithful tracial states. Let Aff $(T(A))$ be the space of all real affine continuous functions on $T(A)$. Denote by $M_{n}(A)$ the matrixes over $A$. By regarding $M_{n}(A)$ as a subset of $M_{n+1}(A)$, define $M_{\infty}(A)=\cup_{n=1}^{\infty} M_{n}(A)$. If $\tau \in T(A)$, then $\tau \otimes \operatorname{Tr}$, where $\operatorname{Tr}$ is standard trace on $M_{n}$, is a trace on $M_{n}(A)$. Throughout this paper, we may use $\tau$ for $\tau \otimes \operatorname{Tr}$ without warning.

If $B$ is another $C^{*}$-algebra and $\varphi: A \rightarrow B$ is a contractive completely positive linear map, then $\varphi \otimes \mathrm{id}_{M_{n}}$ gives a contractive completely positive linear map from $M_{n}(A)$ to $M_{n}(B)$. Throughout this paper, we may use $\varphi$ for $\varphi \otimes \operatorname{id}_{M_{n}}$ for convenience.

Let $C$ and $A$ be two unital $C^{*}$-algebras with $T(C) \neq \emptyset$ and $T(A) \neq \emptyset$. Suppose that $h$ : $C \rightarrow A$ is a unital homomorphism. Define an affine continuous map $h_{\sharp}: T(A) \rightarrow T(C)$ by $h_{\sharp}(\tau)(c)=\tau \circ h(c)$ for all $\tau \in T(A)$ and $c \in C$. If $A$ is simple and $h$ is a monomorphism, then $h_{\sharp}$ maps $T(A)$ into $T_{\mathrm{f}}(C)$.

Definition 2.2. Let $C$ be a unital $C^{*}$-algebra with $T(C) \neq \emptyset$. For each $p \in M_{n}(C)$ define $\check{p}(\tau)=\tau \otimes \operatorname{Tr}(p)$ for all $\tau \in T(A)$, where $\operatorname{Tr}$ is the standard trace on $M_{n}$. This gives positive homomorphism $\rho_{C}: K_{0}(C) \rightarrow \operatorname{Aff}(T(C))$.

2.3. If $A$ is a unital $C^{*}$-algebra with tracial rank at most one, then we will write $T R(A) \leq 1$ (see [12]).

2.4. Let $C$ be a unital $C^{*}$-algebra. Denote by $U(C)$ the unitary group of $C$ and denote by $U_{0}(C)$ the subgroup of $U(C)$ consisting of unitaries which connected to $1_{C}$ by a continuous path of unitaries. Denote by $C U(C)$ be the closure of the normal subgroup generated by commutators of $U_{0}(C)$. Let $u \in U(C)$. Then $\bar{u}$ is the image of $u$ in $U(C) / C U(C)$.

Now suppose that $T(C) \neq \emptyset$. Let $n \geq 1$ be an integer. Let $u \in U_{0}\left(M_{n}(C)\right)$. Let $\gamma \in$ $C\left([0,1], U_{0}\left(M_{n}(C)\right)\right)$ which is piecewise smooth such that $\gamma(1)=u$ and $\gamma(0)=1$. Define

$$
\Delta(\gamma)(\tau)=\frac{1}{2 \pi i} \int_{0}^{1} \tau\left(\frac{d \gamma(t)}{d t} \gamma^{-1}(t)\right) d t
$$

where $\tau$ is identified with $\tau \otimes \operatorname{Tr}$ (in particular, for $n>1, \tau$ in the above formula is not the normalized trace). As in ([27]), the de la Harpe-Scandalis determinant provides a continuous homomorphism

$$
\bar{\Delta}: \cup_{k=1}^{\infty}\left(U_{0}\left(M_{k}(C)\right) / U_{0}\left(M_{k}(C)\right) \cap C U_{0}\left(M_{k}(C)\right) \rightarrow \operatorname{Aff}(T(C)) / \overline{\rho_{C}\left(K_{0}(C)\right)} .\right.
$$

We will use $C U\left(M_{\infty}(C)\right)$ for $\cup_{k=1}^{\infty} C U\left(M_{k}(C)\right)$.

Define a metric as follows. If $u, v \in U\left(M_{n}(C)\right)$ such that $u v^{*} \in U_{0}\left(M_{n}(C)\right)$, define

$$
\operatorname{dist}(\bar{u}, \bar{v})=\left\|\bar{\Delta}\left(u v^{*}\right)\right\| \text {. }
$$

Note that if $u, v \in U_{0}\left(M_{n}(C)\right)$, then

$$
\operatorname{dist}(\bar{u}, \bar{v})=\|\bar{\Delta}(u)-\bar{\Delta}(v)\|
$$

(where the norm is the quotient norm in $\operatorname{Aff}(T(C)) / \overline{\rho_{C}\left(K_{0}(C)\right)}$ ).

Note that if $u \in C U(C)$, then $[u]=0$ in $K_{1}(C)$. Using de la Harpe-Scandalis determinant, by K. Thomsen (see [27]), one has the following short splitting exact sequence:

$$
0 \rightarrow \operatorname{Aff}(T(C)) / \overline{\rho_{C}\left(K_{0}(C)\right)} \rightarrow U\left(M_{\infty}(C)\right) / C U\left(M_{\infty}(C)\right) \rightarrow_{\pi_{C}} K_{1}(C) \rightarrow 0
$$


We will fix one splitting map $J_{C}: K_{1}(C) \rightarrow U\left(M_{\infty}(C)\right) / C U\left(M_{\infty}(C)\right)$ such that $\pi_{C} \circ J_{C}=$ $\operatorname{id}_{K_{1}(C)}$. For each $\bar{u} \in J_{C}\left(K_{1}(C)\right)$, select and fix one element $u_{c} \in \cup_{n=1}^{\infty} M_{n}(C)$ such that $\overline{u_{c}}=\bar{u}$. Denote this set by $U_{c}\left(K_{1}(C)\right)$.

If $A$ is a unital $C^{*}$-algebra and $\varphi: C \rightarrow A$ is a unital homomorphism, then $\varphi$ induces a continuous map

$$
\varphi^{\ddagger}: U\left(M_{\infty}(C)\right) / C U\left(M_{\infty}(C)\right) \rightarrow U\left(M_{\infty}(A)\right) / C U\left(M_{\infty}(A)\right) .
$$

Denote by $\varphi^{\dagger}: K_{1}(C) \rightarrow \operatorname{Aff}(T(A)) / \overline{\rho_{A}\left(K_{0}(A)\right)}$ the map (id $\left.-J_{A} \circ \pi_{A}\right) \circ \varphi^{\ddagger} \circ J_{C}$, where $J_{A}$ : $K_{1}(A) \rightarrow U\left(M_{\infty}(A)\right) / C U\left(M_{\infty}(A)\right.$ is a fixed splitting map. Note that $\varphi^{\dagger}$ depends on the splitting maps, but for each $C^{*}$-algebra, splitting maps are fixed.

If $A$ is a unital simple $C^{*}$-algebra with $T R(A) \leq 1$, by Cor. 3.5 of [18],

$$
U_{0}(C) / C U(C)=U_{0}\left(M_{n}(C)\right) / C U\left(M_{n}(C)\right)
$$

for all $n \geq 1$. Since in this case, $K_{1}(C)=U(C) / U_{0}(C)$, we have isomorphism between $U(C) / C U(C)$ and $U\left(M_{\infty}(C)\right) / C U\left(M_{\infty}(C)\right)$.

2.5. Let $A$ be a unital $C^{*}$-algebra and let $u \in U_{0}(A)$. Let $\gamma \in C([0,1], U(A))$ such that $\gamma(0)=1$ and $\gamma(1)=u$. Denote by Length $(\{\gamma\})$ the length of the path $\gamma$. Put

$$
\operatorname{cel}(u)=\inf \{\text { Length } \gamma: \gamma \in C([0,1], U(A)), \gamma(0)=1 \text { and } \gamma(1)=u\} .
$$

Definition 2.6. Let $C$ be a $C^{*}$-algebra and let $\mathcal{P} \subset \underline{K}(C)$. There exists $\delta>0$ and a finite subset $\mathcal{G} \subset C$ such that, for any $\delta$ - $\mathcal{G}$-multiplicative contractive completely positive linear map $L: C \rightarrow A$ (for any $C^{*}$-algebra $A$ ), $\left.[L]\right|_{\mathcal{P}}$ is well defined (see 0.6 of [10] and 2.3 of [17] ). Such a triple $(\delta, \mathcal{G}, \mathcal{P})$ is called local $\underline{K}$-triple (see [3]). If $K_{i}(C)$ is finitely generated $(i=0,1)$ and $\mathcal{P}$ is large enough, then $\left.[L]\right|_{\mathcal{P}}$ defines an element in $K K(C, A)$ (see 2.4 of [17]). In such cases, we will write $[L]$ instead of $\left.[L]\right|_{\mathcal{P}}$, and we will call $(\delta, \mathcal{G}, \mathcal{P})$ a $K K$-triple and $(\delta, \mathcal{G})$ a $K K$-pair. Note that, if $u$ is a unitary then, we write $\langle L(u)\rangle=L(u)\left(L(u)^{*} L(u)\right)^{-1 / 2}$ when $\left\|L\left(u^{*}\right) L(u)-1\right\|<1$ and $\left\|L(u) L\left(u^{*}\right)-1\right\|<1$. In what follows we will always assume that $\left\|L\left(u^{*}\right) L(u)-1\right\|<1$ and $\left\|L(u) L\left(u^{*}\right)-1\right\|<1$, when we write $\langle L(u)\rangle$.

Suppose that $C$ is a separable $C^{*}$-algebra and $C$ is the closure of $\cup_{n=1}^{\infty} C_{n}$, where each $C_{n}=\lim _{m \rightarrow \infty}\left(C_{n, m}, \varphi_{m}^{(n)}\right)$ and $K_{i}\left(C_{n, m}\right)$ is finitely generated $(i=0,1)$. Denote by $\imath_{n}: C_{n} \rightarrow C$ the embedding and $\varphi_{m, \infty}^{(n)}: C_{n, m} \rightarrow C_{n}$ the homomorphism induced by the inductive system $\left(C_{n, m}, \varphi_{m}\right)$. We say that $(\delta, \mathcal{G}, \mathcal{P})$ is a $K L$-triple, if $\left[\imath_{n} \circ \varphi_{m, \infty}^{(n)}\right]\left(\mathcal{P}^{\prime}\right) \supset \mathcal{P}$ for some $n, m$ and some finite subset $\mathcal{P}^{\prime} \subset \underline{K}\left(C_{n, m}\right)$ and if any $\delta$ - $\mathcal{G}$-multiplicative contractive completely positive linear map $L: C \rightarrow A$ (for any $A$ ) gives a $\delta$ - $\mathcal{G}^{\prime}$-multiplicative contractive completely positive linear map $L \circ \imath_{n} \circ \varphi_{m, \infty}^{(n)}$ so that $\left(\delta, \mathcal{G}^{\prime}, \mathcal{P}^{\prime}\right)$ is a $K K$-triple.

Proposition 2.7. Let $A$ be a (unital) separable amenable $C^{*}$-algebra and $\left\{I_{n}\right\}$ be a sequence of increasing closed two sided ideals of $A$. Let $I=\overline{\cup_{n=1}^{\infty} I_{n}}$ and $B=A / I$. Suppose that $\mathcal{F} \subset B$ is a finite subset and $\epsilon>0$. Then there exists an integer $n \geq 1$ and a (unital) contractive completely positive linear map $L: B \rightarrow A / I_{n}$ which is $\epsilon-\mathcal{F}$-multiplicative such that $\pi_{n} \circ L(b)=b$ for all $b \in B$, where $\pi_{n}: A / I_{n} \rightarrow A / I$ is the quotient map.

Proof. By the Efros-Choi Lifting Theorem, there is a (unital) contractive completely positive linear map $L_{0}: B \rightarrow A$ such that $\pi \circ L_{0}=\operatorname{id}_{B}$, where $\pi: A \rightarrow A / I$ is the quotient map. For each pair $a, b \in B$, let $c(a, b)=L_{0}(a b)-L_{0}(a) L_{0}(b)$. Then

$$
\pi(c(a, b))=\pi\left(L_{0}(a b)\right)-\pi\left(L_{0}(a) L_{0}(b)\right)=0 .
$$


It follows that $c(a, b) \in I$. Therefore there exists an integer $n \geq 1$ such that

$$
\left\|c(a, b)-I_{n}\right\|<\epsilon \text { for all } a, b \in \mathcal{F} \text {. }
$$

Let $\pi_{n}: A \rightarrow A / I_{n}$ be the quotient map and $L=\pi_{n} \circ L_{0}: B \rightarrow A / I_{n}$. Then

$$
\|L(a b)-L(a) L(b)\|=\| \pi_{n}(c(a, b) \|<\epsilon \text { for all } a, b \in \mathcal{F} .
$$

Definition 2.8. Let $X$ be a compact metric space, let $x \in X$ and let $r>0$. Denote by $O(x, r)$ the open ball with center at $x$ and radius $r$. If $x$ is not specified $O(r)$ is an open ball of radius $r$.

Definition 2.9. Let $X$ be a metric space and $s: C(X) \rightarrow \mathbb{C}$ be a state. Denote by $\mu_{s}$ the probability Borel measure induced by $s$.

The following could be proved directly but also follows from 4.6 of [14].

Theorem 2.10. Let $X$ be a compact metric space, let $\epsilon>0$ and let $\mathcal{F} \subset C(X)$ be a finite subset. There exists $\eta>0$ satisfying the following: for any $\sigma>0$, there exists $\gamma>0, \delta>0$, a finite subset $\mathcal{G} \subset C(X)$ and a finite subset $\mathcal{H} \subset C(X)_{\text {s.a }}$ and a finite subset $\mathcal{P} \subset \underline{K}(C(X))$ satisfying the following:

For any unital $\delta$-G-multiplicative contractive completely positive linear maps $\varphi, \psi: C(X) \rightarrow$ $M_{n}$ (for some integer $n \geq 1$ ) for which

$$
\left.[\varphi]\right|_{\mathcal{P}}=\left.[\psi]\right|_{\mathcal{P}}, \quad \mu_{\tau \circ \varphi}\left(O_{r}\right) \geq \sigma
$$

for all open balls $O_{r}$ of radius $r \geq \eta$ and

$$
|\tau \circ \varphi(a)-\tau \circ \psi(a)|<\gamma \text { for all } a \in \mathcal{H}
$$

where $\tau$ is the tracial state of $M_{n}$, there is a unitary $u \in M_{n}$ such that

$$
\|\varphi(f)-\operatorname{Ad} u \circ \psi(f)\|<\epsilon \text { for all } f \in \mathcal{F} \text {. }
$$

The following is also known and could be derived from the above.

Corollary 2.11. Let $X$ be a compact metric space, let $\epsilon>0$ and let $\mathcal{F} \subset C(X)$ be a finite subset, there exists $\eta>0$ satisfying the following: For any $\sigma>0$, there exists $\gamma>0$, a finite subset $\mathcal{P} \subset K_{0}(C(X))$ and a finite subset $\mathcal{H} \subset C(X)_{\text {s.a. satisfying the following: For any two }}$ unital homomorphisms $\varphi, \psi: C(X) \rightarrow M_{n}$ (for some integer $n \geq 1$ ) such that

$$
\left.[\varphi]\right|_{\mathcal{P}}=\left.[\psi]\right|_{\mathcal{P}}, \quad \mu_{\tau \circ \varphi}\left(O_{r}\right) \geq \sigma
$$

for all open ball $O_{r}$ of $X$ with radius $r \geq \eta$ and

$$
|\tau \circ \varphi(a)-\tau \circ \psi(a)|<\gamma \text { for all } a \in \mathcal{H},
$$

there exists a unitary $u \in M_{n}$ such that

$$
\|\operatorname{Ad} u \circ \varphi(f)-\psi(f)\|<\epsilon \text { for all } f \in \mathcal{F} \text {. }
$$

The following is a variation of Lemma 4.3 of [21]. 
Corollary 2.12. Let $X$ be a compact metric space, $\epsilon>0$ and $\mathcal{F} \subset C(X)$ be a finite subset. There exists $\eta_{1}>0$ satisfying the following: for any $\sigma_{1}>0$ and any $0<\lambda<1$, there exists $\eta_{2}>0$ satisfying the following: for any $\sigma_{2}>0$, there exists $\delta>0$, a finite subset $\mathcal{G} \subset C(X)$ and a finite subset $\mathcal{P} \subset \underline{K}(C(X))$ satisfying the following:

For any unital $\delta-\mathcal{G}$-multiplicative contractive completely positive linear map $\varphi: C(X) \rightarrow M_{n}$ (for some integer $n \geq 1$ ) such that

$$
\left.[\varphi]\right|_{\mathcal{P}}=\left.[H]\right|_{\mathcal{P}}
$$

for some unital homomorphism $H: C(X) \rightarrow M_{n}$ and such that

$$
\mu_{\tau \circ \varphi}\left(O_{r}\right) \geq \sigma_{1} \text { and } \mu_{\tau \circ \varphi}\left(O_{r}\right) \geq \sigma_{2}
$$

for all open balls $O_{r}$ of radius $r \geq \eta_{1}$ and $r \geq \eta_{2}$, respectively, there is a unital homomorphism $h: C(X) \rightarrow M_{n}$ such that

$$
\|\varphi(f)-h(f)\|<\epsilon \text { for all } f \in \mathcal{F} \text {. }
$$

Moreover,

$$
\mu_{t r \circ h}\left(O_{r}\right) \geq \lambda \sigma_{1}
$$

for all $r \geq 2 \eta_{1}$.

2.13. Let $C$ be a unital $C^{*}$-algebra and let $\mathcal{P} \subset \underline{K}(C)$ be a finite subset. There is a finite subset $\mathcal{F}_{C, \mathcal{P}, \mathbf{b}} \subset C$ and a positive number $\delta_{C, \mathcal{P}, \mathbf{b}}>0$ such that $\left.\operatorname{Bott}(u, h)\right|_{\mathcal{P}}$ (see the definition 2.10 of [17]) is well defined for any unital $C^{*}$-algebra $A$, any unital homomorphism $h: C \rightarrow A$ and any unitary $u \in A$ for which

$$
\|[h(f), u]\|<\delta_{C, \mathcal{P}, \mathbf{b}} \text { for all } f \in \mathcal{F}_{C, \mathcal{P}}
$$

Moreover, by choosing even smaller $\delta_{C, \mathcal{P}, \mathbf{b}}$, if $h_{1}: C \rightarrow A$ is another unital homomorphism and

$$
\left\|h(f)-h_{1}(f)\right\|<\delta_{C, \mathcal{P}, \mathbf{b}},
$$

then $\left.\operatorname{Bott}\left(u, h_{1}\right)\right|_{\mathcal{P}}$ is also well defined and

$$
\left.\operatorname{Bott}(u, h)\right|_{\mathcal{P}}=\left.\operatorname{Bott}\left(u, h_{1}\right)\right|_{\mathcal{P}}
$$

As in tradition,

$$
\left.\operatorname{bott}_{1}(u, h)\right|_{\mathcal{P}}=\left.\operatorname{Bott}(u, h)\right|_{\mathcal{P} \cap K_{1}(C)} \text { and }\left.\operatorname{bott}_{0}(u, h)\right|_{\mathcal{P}}=\left.\operatorname{Bott}(u h)\right|_{\mathcal{P} \cap K_{0}(C)} .
$$

If $K_{i}(C)(i=0,1)$ is finitely generated, then, by choosing $\mathcal{P}$ large enough, we may assume that, when (e e 2.16$)$ holds, $\operatorname{Bott}(h, u)$ is well defined. Furthermore, we will write $\delta_{C, \mathbf{b}}$ instead of $\delta_{C, \mathcal{P}, \mathbf{b}}$ and $\mathcal{F}_{C, \mathbf{b}}$ instead of $\mathcal{F}_{C, \mathcal{P}, \mathbf{b}}$.

If $C=C(\mathbb{T})$, let $z \in U(C(\mathbb{T}))$ be the standard unitary generator, one writes that

$$
\operatorname{bott}_{1}(u, h)=\operatorname{bott}(u, h(z)) .
$$

Suppose that there is a continuous path of unitaries $u(t):[0,1] \rightarrow U_{0}(A)$ such that

$$
u(0)=u, u(1)=1_{A} \text { and }\|[h(f), u(t)]\|<\delta_{C, \mathcal{P}, \mathbf{b}} \text { for all } t \in[0,1]
$$


then

$$
\left.\operatorname{Bott}(u, h)\right|_{\mathcal{P}}=0 .
$$

Now suppose that $C$ is a unital separable amenable $C^{*}$-algebra which is the closure of $\cup_{n=1}^{\infty} C_{n}$, where $C_{n}=\lim _{n \rightarrow \infty}\left(C_{n, m}, \varphi_{m}^{(n)}\right)$ and $K_{i}\left(C_{n, m}\right)$ is finitely generated $(i=0,1)$. Let $z$ be the standard unitary generator of $C(\mathbb{T})$. We may view $\mathcal{P}$ as a subset of $\underline{K}(C \otimes C(\mathbb{T}))$. Let $\mathcal{G}_{0}$ be a finite subset of $C$. Define $\mathcal{G}_{1}=\left\{g \otimes f: g \in \mathcal{G}_{0}\right.$ and $\left.f \in \mathcal{S}\right\}$, where $S=\left\{1, z, z^{*}\right\}$. Let $\mathcal{P}_{1}=\mathcal{P} \cup \boldsymbol{\beta}(\mathcal{P})$ (see 2.10 of [17] for the definition of $\boldsymbol{\beta}$ ). Let $\delta>0$. Suppose that $\left(\delta, \mathcal{G}_{1}, \mathcal{P}_{1}\right)$ is a $K L$-triple for $C \otimes C(\mathbb{T})$ (by selecting large $\mathcal{G}_{0}$ to begin with).

By choosing even smaller $\delta_{C, \mathcal{P}, \mathbf{b}}$, we may assume that, if there is a unitary $u \in A$ such that (e2.16) holds, and if there is a unital $\delta$ - $\mathcal{G}_{1}$-multiplicative contractive completely positive linear $\operatorname{map} L: C \otimes C(\mathbb{T}) \rightarrow A$ such that

$$
\begin{aligned}
\|L(f \otimes 1)-h(f)\| & <\delta_{C, \mathcal{P}, \mathbf{b}} \text { for all } f \in \mathcal{F}_{C, \mathcal{P}, \mathbf{b}} \\
\text { and }\|u-L(1 \otimes z)\| & <\delta_{C, \mathcal{P}, \mathbf{b}},
\end{aligned}
$$

then

$$
\left.\operatorname{Bott}(u, h)\right|_{\mathcal{P}}=\left.[L]\right|_{\boldsymbol{\beta}(\mathcal{P})} .
$$

The following is a restatement of Theorem 7.4 of [17].

Theorem 2.14. Let $X$ be a compact metric space. For any $\epsilon>0$ and any finite subset $\mathcal{F} \subset$ $C(X)$, there exists $\eta>0$ satisfying the following: For any $\sigma>0$, there exists $\delta>0$, a finite subset $\mathcal{G} \subset C(X)$ and a finite subset $\mathcal{P} \subset \underline{K}(C(X))$ satisfying the following: Suppose that $\varphi: C(X) \rightarrow M_{n}$ is a unital homomorphism such that

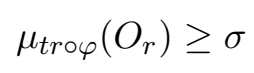

for all open ball $O_{r}$ with radius $r \geq \eta$. If $u \in M_{n}$ is a unitary such that

$$
\|[u, \varphi(g)]\|<\delta \text { for all } g \in \mathcal{G} \text { and }\left.\operatorname{Bott}(h, u)\right|_{\mathcal{P}}=0,
$$

then there exists a continuous rectifiable path of unitaries $\left\{u_{t}: t \in[0,1]\right\}$ of $M_{n}$ such that

$$
u_{0}=1, u_{1}=1_{A} \text { and }\left\|\left[h(f), u_{t}\right]\right\|<\epsilon
$$

for all $f \in \mathcal{F}$ and $t \in[0,1]$. Moreover,

$$
\operatorname{Length}\left(\left\{u_{t}\right\}\right) \leq 2 \pi+\epsilon \pi
$$

\section{Almost multiplicative maps from $C(X)$ into interval algebras}

Lemma 3.1. Let $X$ be a compact metric space. For any $\epsilon>0$, any finite subset $\mathcal{F} \subset C(X)$ and any finite subset $\mathcal{P} \subset \underline{K}(C(X))$, there exists a finite subset $\left\{s_{1}, s_{2}, \ldots, s_{k(X)}\right\} \subset K_{1}(C(X))$ and $\eta>0$ satisfying the following: For any $1>\sigma>0$, there exists $d>0$, for any $\alpha \in$ $K L(C(X) \otimes C(\mathbb{T}), \mathbb{C})=H_{\Lambda}(\underline{K}(C(X) \otimes C(\mathbb{T}), \underline{K}(\mathbb{C}))$ and for any unital homomorphism $\varphi: C(X) \rightarrow M_{n}$ for some integer $n \geq 1$ for which

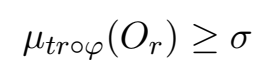

for any open balls $O_{r}$ with radius $r \geq \eta$, where $t r$ is the normalized trace on $M_{n}$, and

$$
\max \left\{\left|\alpha \circ \beta^{(1)}\left(s_{i}\right)\right|: 1 \leq i \leq k(X)\right\} / n<d,
$$

there exists a unitary $u \in M_{n}$ such that

$$
\|[\varphi(f), u]\|<\epsilon \text { for all } f \in \mathcal{F} \text { and }\left.\operatorname{Bott}(\varphi, u)\right|_{\mathcal{P}}=\left.\alpha\right|_{\boldsymbol{\beta}(\mathcal{P})} .
$$


(Note that, here $\beta^{(1)}: K_{1}\left(C(C(X)) \rightarrow K_{0}(C(X) \otimes C(\mathbb{T}))\right)$ and $\boldsymbol{\beta}: \underline{K}(C(X)) \rightarrow \underline{K}(C(X) \otimes$ $C(\mathbb{T}))$ are as defined in 2.10 of [17].)

Proof. Let $\epsilon>0, \mathcal{F} \subset C(X)$ and $\mathcal{P} \subset \underline{K}(C(X))$ be finite subsets. Let $\epsilon_{1}=\min \left\{\epsilon / 2, \delta_{C(X), \mathcal{P}, \mathbf{b}}\right\}$ and let $\mathcal{F}_{1}=\mathcal{F} \cup \mathcal{F}_{C(X), \mathcal{P}, \mathbf{b}}$.

Let $\eta^{\prime}>0$ (in place of $\eta$ ) be given by 2.10 associated with $\epsilon / 16$ (in place of $\epsilon$ ) and $\mathcal{F}$. Let $1>\sigma>0$. Let $\gamma>0, \delta>0, \mathcal{G}, \mathcal{P}_{1} \subset \underline{K}(C(X))$ (in place of $\mathcal{P}$ ) and $\mathcal{H} \subset C(X)$ be given by 2.10 associated with the above $\epsilon / 16$ (in place of $\epsilon$ ), $\eta_{1}>0$ (in place of $\eta$ ) and $\sigma / 2$. We may assume that $\mathcal{P} \subset \mathcal{P}_{1}$.

For convenience, we may assume that $\mathcal{H} \cup \mathcal{F} \subset \mathcal{G}$. We may assume that $\delta<\min \{\epsilon / 2,1 / 4\}$, $\|g\| \leq 1$ if $g \in \mathcal{G}$ and $1_{C(X)} \in \mathcal{G}$.

Let $\mathcal{G}_{1}=\left\{g \otimes f: g \in \mathcal{G}\right.$ and $\left.f=1, z, z^{*}\right\} \subset C(X) \otimes C(\mathbb{T})$, where $z$ is the identity function on the unit circle. We may also assume that $\left(\delta, \mathcal{G}_{1}, \mathcal{P}_{1}\right)$ is a $K L$-triple for $C(X) \otimes C(\mathbb{T})$. Moreover, we may assume that $\delta<\delta_{C(X), \mathcal{P}, \mathbf{b}}$ and $\mathcal{G} \supset \mathcal{F}_{C(X), \mathbf{b}}$.

Suppose that $C(X)=\lim _{n \rightarrow \infty} C\left(Y_{n}\right)$, where each $Y_{n}$ is a finite CW complex. Let $\imath_{m}$ : $C\left(Y_{m}\right) \rightarrow C(X)$ be the unital homomorphism induced by the inductive limit system. We may assume that $\imath_{m}$ is injective (see [23]). Let $s: X \rightarrow Y_{m}$ be the surjective map such that $\imath(f)(x)=f(s(x))$ for all $f \in C\left(Y_{m}\right)$ and $x \in X$. By choosing a large $m$, we may assume that there is a finite subset $\mathcal{G}^{\prime} \subset C\left(Y_{m}\right)$ and there is a finite subset $\mathcal{P}^{\prime} \subset \underline{K}\left(C\left(Y_{m}\right)\right)$ such that $\imath_{m}\left(\mathcal{G}^{\prime}\right) \supset \mathcal{G}$ and $\left[\imath_{m}\right]\left(\mathcal{P}^{\prime}\right) \supset \mathcal{P}$. Let $\mathcal{G}_{1}^{\prime}=\left\{g \otimes f: g \in \mathcal{G}^{\prime}\right.$ and $\left.f=1, z, z^{*}\right\}$. We may further assume that $\left(2 \delta, \mathcal{G}^{\prime}\right)$ is a $K K$-pair for $C\left(Y_{m}\right) \otimes C(\mathbb{T})$.

Let $\left\{s_{1}^{\prime}, s_{2}^{\prime}, \ldots, s_{k(X)}^{\prime}\right\} \subset K_{1}\left(C\left(Y_{m}\right)\right)$ be a generating subset. Let $s_{i}=\left(\imath_{m}\right)_{* 1}\left(s_{i}\right), i=$ $1,2, \ldots, k(X)$.

Suppose that $Y_{m}$ is the disjoint union of finitely many connected CW complexes $Z_{1}, Z_{2}, \ldots, Z_{l}$. Without loss of generality, we may assume that there is, for each $i$, a finite subset $\mathcal{G}^{(i)} \subset C\left(Z_{i}\right)$ such that $\oplus_{i=1}^{l} \mathcal{G}^{(i)}=\mathcal{G}^{\prime}$ and there is a finite subset $\mathcal{P}_{i}^{\prime} \subset \underline{K}\left(C\left(Z_{i}\right)\right)$ such that $\oplus_{i=1}^{l} \mathcal{P}_{i}^{\prime}=\mathcal{P}^{\prime}$. Choose $\xi_{i} \in Z_{i}$ such that $\xi_{i} \in Y_{m}, i=1,2, \ldots, l$. We assume also that each $Z_{i}$ contains an open ball with radius $r \geq \eta_{0}>0$. Put $\eta=\min \left\{\eta_{0} / 2, \eta^{\prime}\right\}$.

Let $N\left(\delta / 4, \mathcal{G}_{1}^{(i)}, \mathcal{P}_{i}^{\prime}\right)$ be given by Lemma 10.2 of [21] for $C\left(\mathbb{T} \times Z_{i}\right)$. Define

$$
N\left(\delta / 4, \mathcal{G}_{1}^{\prime}, \mathcal{P}^{\prime}\right)=\sum_{i=1}^{l} N\left(\delta / 4, \mathcal{G}_{1}^{(i)}, \mathcal{P}_{i}^{\prime}\right) .
$$

Let

$$
d=\min \{\sigma / 2, \gamma\} \cdot \frac{1}{N\left(\delta / 4, \mathcal{G}_{1}^{\prime}, \mathcal{P}^{\prime}\right)} .
$$

Let $\alpha$ be as in the statement and let

$$
k=\max \left\{\left|\alpha \circ \beta^{(1)}\left(s_{i}\right)\right|: i=1,2, \ldots, k(X)\right\} .
$$

Now suppose that $\varphi: C(X) \rightarrow M_{n}$ for some integer $n \geq 1$ for which

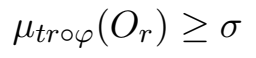

for all open balls $O_{r}$ with radius $r \geq \eta$, where $t r$ is the normalized tracial state on $M_{n}$ and

$$
k / n<d .
$$

We may write that

$$
\varphi(f)=\sum_{i=1}^{n} f\left(\zeta_{i}\right) p_{i} \text { for all } f \in C(X)
$$


where $\left\{p_{1}, p_{2}, \ldots, p_{n}\right\}$ is a set of mutually orthogonal rank one projections and $\zeta_{i} \in X, i=$ $1,2, \ldots, n$. Put $L=k N\left(\delta, \mathcal{G}_{1}^{\prime}, \mathcal{P}^{\prime}\right)$. Note $n>L$.

Note that, if $x \in \operatorname{ker} \rho_{C\left(Y_{m}\right)}$, then $h_{* 0}(x)=0$ for any homomorphism $h: C\left(Y_{m}\right) \rightarrow M_{n}$ (for any integer $n \geq 1)$. Define $H: C\left(Y_{m}\right) \rightarrow M_{L}$ by $H(f)=\sum_{i=1}^{L} f\left(s\left(\zeta_{i}\right)\right) p_{i}$ for $f \in C\left(Y_{m}\right)$.

Choose $\beta \in \operatorname{Hom}_{\Lambda}\left(\underline{K}\left(C\left(Y_{m}\right) \otimes C(\mathbb{T})\right), \underline{K}(\mathbb{C})\right)$ defined as

$$
\left.\beta\right|_{\underline{K}\left(C\left(Y_{m}\right)\right)}=[H]
$$

and

$$
\left.\beta\right|_{\boldsymbol{\beta}\left(\underline{K}\left(C\left(Y_{m}\right)\right)\right)}=\left.\alpha \circ\left[\imath_{m}\right]\right|_{\boldsymbol{\beta}\left(\underline{K}\left(C\left(Y_{m}\right)\right)\right)}
$$

(see 2.10 of [17] for the definition of $\boldsymbol{\beta}$ ). Let $\mathcal{G}_{1}=\{g \otimes 1: g \in \mathcal{G}\} \cup\left\{z_{1}\right\} \subset C\left(Y_{m}\right) \otimes C(\mathbb{T})$, where $z_{1}=1 \otimes z$ and $z$ is the identity function on the unit circle.

It follows from Lemma 10.2 of [21] (and considering each component of $Y_{m}$ separately) that there exists a unital $\delta / 4-\mathcal{G}_{1}^{\prime}$-multiplicative contractive completely positive linear map $\Phi^{\prime}$ : $C\left(Y_{m}\right) \otimes C(\mathbb{T}) \rightarrow M_{L}$ such that

$$
\left[\Phi^{\prime}\right]=\beta
$$

One obtains a $\delta / 2-\mathcal{G}$-multiplicative contractive completely positive linear map $\Phi: C(X) \otimes$ $C(\mathbb{T}) \rightarrow M_{L}$ such that

$$
\left\|\Phi\left(\imath_{m}(g) \otimes f\right)-\Phi^{\prime}(g \otimes f)\right\|<\delta / 2 \text { for all } g \in \mathcal{G}^{\prime \prime} \text { and } f \in\left\{1, z, z^{*}\right\},
$$

where $\mathcal{G}^{\prime \prime} \subset \mathcal{G}$ such that $\imath_{m}(g) \in \mathcal{G}$. Furthermore

$$
\begin{aligned}
{\left.[\Phi]\right|_{\mathcal{P}_{1}} } & =\left.\left[H_{1}\right]\right|_{\mathcal{P}_{1}}\left(\mathcal{P}_{1} \subset \underline{K}(C(X))\right) \\
\text { and }\left.[\Phi]\right|_{\boldsymbol{\beta}(\mathcal{P})} & =\left.\alpha\right|_{\boldsymbol{\beta}(\mathcal{P})},
\end{aligned}
$$

where $H_{1}: C(X) \rightarrow M_{L}$ is defined by $H_{1}(f)=\sum_{i=1}^{L} f\left(\zeta_{i}\right) p_{i}$ for $f \in C(X)$.

Define $\varphi_{0}^{\prime}: C(X) \rightarrow M_{L}$ by $\varphi_{0}^{\prime}(f)=\Phi\left(f \otimes 1_{C(\mathbb{T})}\right)$ for all $f \in C(X)$. Define

$$
u_{0}=\Phi\left(1_{C(X)} \otimes z\right)\left(\Phi\left(1_{C(X)} \otimes z^{*}\right) \Phi\left(1_{C(X)} \otimes z\right)\right)^{1 / 2}=\left\langle\Phi\left(1_{C(X)} \otimes z\right)\right\rangle .
$$

Then

$$
\left\|u_{0}-\Phi\left(1_{C(X)} \otimes z\right)\right\|<\delta_{C(X), \mathcal{P}, \mathbf{b}} .
$$

Define $\varphi^{\prime}: C(X) \rightarrow M_{n-L}$ defined by

$$
\varphi^{\prime}(f)=\sum_{i=L+1}^{n} f\left(\zeta_{i}\right) p_{i} \text { for all } f \in C(X) .
$$

Define $\varphi_{1}: C(X) \rightarrow M_{n}$ by

$$
\varphi_{1}(f)=\varphi^{\prime}(f) \oplus \varphi_{0}(f) \text { for all } f \in C(X) .
$$

Since $k / n<d \leq \gamma(k / L), L / n<\gamma$. Therefore one computes that

$$
\begin{aligned}
& {\left.\left[\varphi_{1}\right]\right|_{\mathcal{P}_{1}}=\left.\left[\varphi_{2}\right]\right|_{\mathcal{P}_{1}} \text { and }} \\
& \left|\tau \circ \varphi(g)-\tau \circ \varphi_{1}(f)\right|<\gamma \text { for all } g \in \mathcal{H} .
\end{aligned}
$$

Moreover, since $k / n<d \leq(\sigma / 2) k / L, L / n<\sigma / 2$. Therefore, by (e3.24),

$$
\mu_{t r \circ \varphi_{1}}\left(O_{r}\right) \geq \sigma / 2 \text { and } \mu_{t r \circ \varphi}\left(O_{r}\right) \geq \sigma / 2
$$


for all $r \geq \eta$.

It follows from 2.10 and 2.11 that there is a unitary $w_{1} \in M_{n}$ such that

$$
\left\|\operatorname{Ad} w_{1} \circ \varphi_{1}(f)-\varphi(f)\right\|<\epsilon / 4 \text { for all } f \in \mathcal{F} \text {. }
$$

Put

$$
u=w^{*}\left(\operatorname{diag}(\overbrace{1,1, \ldots, 1}^{n-L}, u_{0})\right) w .
$$

One checks that this unitary $u$ meets all the requirements.

The following is a folklore.

Lemma 3.2. Let $X$ be a compact metric space, let $\eta_{i}>0$ and $\sigma_{i}>0(i=1,2, \ldots, m)$ with $\eta_{1}>\eta_{2}>\cdots>\eta_{m}$ and $\sigma_{1}>\sigma_{2}>\cdots>\sigma_{m}$, and let $0<\lambda_{1}, \lambda_{2}<1$. There exists $\delta>0$ and $a$ finite subset $\mathcal{G} \subset C(X)$ satisfying the following:

Suppose that $A$ is a unital $C^{*}$-algebra with $T(A) \neq \emptyset$ and suppose that $\varphi, \psi: C(X) \rightarrow A$ are two unital positive linear maps such that

$$
\mu_{\tau \circ \varphi}\left(O_{r}\right) \geq \sigma_{j}
$$

for all $r \geq \eta_{j}, j=1,2, \ldots, m$, and

$$
|\tau \circ \varphi(g)-\tau \circ \psi(g)|<\delta \text { for all } g \in \mathcal{G} .
$$

for all $\tau \in T(A)$. Then,

$$
\mu_{\tau \circ \psi}\left(O_{r}\right) \geq \lambda_{1} \sigma_{j}
$$

for all $r \geq 2\left(1+\lambda_{2}\right) \eta_{j}, j=1,2, \ldots, m$, and for all $\tau \in T(A)$.

Proof. To simplify the proof, without loss of generality, we will prove only for the case that $m=1$. The general case follows by taking minimum of $m \delta$ 's and the union of $m \mathcal{G}^{\prime} s$.

There are $x_{1}, x_{2}, \ldots, x_{K} \in X$ such that

$$
\cup_{k=1}^{K} O\left(x_{k}, \eta\right) \supset X .
$$

There are $f_{1}, f_{2}, \ldots, f_{K} \in C(X)$ with $0<f_{k} \leq 1$ such that $f_{k}(x)=1$ if $x \in O\left(x_{k}, \eta\right)$ and $f_{k}(x)=0$ if $\operatorname{dist}\left(x, x_{k}\right)>\left(1+\lambda_{2}\right) \eta$. Choose $\delta=\left(1-\lambda_{1}\right) \sigma_{1}$ and $\mathcal{G}=\left\{f_{1}, f_{2}, \ldots, f_{K}\right\}$.

Now suppose that $\varphi, \psi: C(X) \rightarrow A$ are two unital positive linear maps which satisfy the assumption (e3.38) and (e3.39).

Let $x \in X$ and consider $O(x, r)$ for some $r \geq 2\left(1+\lambda_{2}\right) \eta$. Then there exists $x_{k}$ such that $\operatorname{dist}\left(x, x_{k}\right)<\eta$. This implies that

$$
O\left(x_{k},\left(1+\lambda_{2}\right) \eta\right) \subset O(x, r) .
$$

Thus

$$
\begin{aligned}
\mu_{\tau \circ \psi}(O(x, r)) & \geq \tau \circ \psi\left(f_{k}\right)>\tau \circ \varphi\left(f_{k}\right)-\left(1-\lambda_{1}\right) \sigma_{1} \\
& \geq \mu_{\tau \circ \varphi}\left(O\left(x_{k}, \eta\right)\right)-\left(1-\lambda_{1}\right) \sigma_{1} \\
& \geq \lambda_{1} \sigma_{1} .
\end{aligned}
$$

for all $\tau \in T(A)$. 
Remark 3.3. Note that in the above lemma, we insist that $\delta$ and $\mathcal{G}$ do not depend on $\varphi$. Otherwise one can have better estimates.

Lemma 3.4. Let $X$ be a compact metric space, let $\Delta:(0,1) \rightarrow(0,1)$ be a nondecreasing function, let $\eta>0$ and let $0<\lambda_{1}, \lambda_{2}<1$. There exists $\delta>0$ and a finite subset $\mathcal{G} \subset C(X)$ satisfying the following:

Suppose that $A$ is a unital $C^{*}$-algebra with $T(A) \neq \emptyset$ and suppose that $\varphi, \psi: C(X) \rightarrow A$ are two unital positive linear maps such that

$$
\mu_{\tau \circ \varphi}\left(O_{r}\right) \geq \Delta(r)
$$

for all $r \geq \eta$ and

$$
|\tau \circ \varphi(g)-\tau \circ \psi(g)|<\delta \text { for all } g \in \mathcal{G} \text {. }
$$

Then,

$$
\mu_{\tau \circ \psi}\left(O_{r}\right) \geq \lambda_{1} \Delta\left(r / 2\left(1+\lambda_{2}\right)\right)
$$

for all $r \geq 2\left(1+\lambda_{2}\right) \eta$.

Proof. Let $\eta>0, \Delta$ and $0<\lambda_{1}, \lambda_{2}<1$ be given. Choose $\lambda_{0}>0$ such that $0<\lambda_{0}<\lambda_{2}$. Let $1>r_{1}>r_{2}>\cdots>r_{N}>0$ such that $\eta>r_{N}$ and

$$
r_{i+1} / r_{i}>\frac{1+\lambda_{0}}{1+\lambda_{2}}, \quad i=1,2, \ldots, N-1 .
$$

Put $\eta_{j}=r_{j}$ and $\sigma_{j}=\Delta\left(\eta_{j}\right), j=1,2, \ldots, N-1$.

Let $\delta>0$ and $\mathcal{G}$ be required by 3.2 for $\eta_{j}$ and $\sigma_{j}(j=1,2, \ldots, N), \lambda_{1}$ and $\lambda_{2}$.

Now suppose that $\varphi, \psi$ satisfy (e 3.43$)$ and (e3.44). By applying 3.2 , we conclude that

$$
\mu_{\tau \circ \psi}\left(O_{r}\right) \geq \lambda_{1} \sigma_{j}
$$

for all $\tau \in T(A)$ and all $r \geq 2\left(1+\lambda_{0}\right) \eta_{j}, j=1,2, \ldots, N$.

Now suppose that $r \geq 2\left(1+\lambda_{2}\right) \eta>2\left(1+\lambda_{0}\right) \eta$. Then

$$
\frac{r}{2\left(1+\lambda_{0}\right)}>\eta
$$

We may assume that, for some $j$,

$$
\eta_{j}>\frac{r}{2\left(1+\lambda_{0}\right)}>\eta_{j+1}
$$

Then

$$
\begin{aligned}
\mu_{\tau \circ \psi}\left(O_{r}\right) & >\lambda_{1} \sigma_{j+1}=\lambda_{1} \Delta\left(\eta_{j+1}\right) \\
& \geq \lambda_{1} \Delta\left(\eta_{j}\left(\frac{1+\lambda_{0}}{1+\lambda_{2}}\right)\right) \\
& \geq \lambda_{1} \Delta\left(\frac{r}{2\left(1+\lambda_{2}\right)}\right)
\end{aligned}
$$

for all $\tau \in T(A)$. 
Lemma 3.5. Let $u \in C U\left(M_{n}(C([0,1]))\right.$ be a unitary such that

$$
\left\|u(0) u(t)^{*}-1\right\|<1 \text { for all } t \in[0,1] .
$$

Suppose that $u(0) u(1)^{*}=\exp (\sqrt{-1} h)$ with $\|h\|<2 \arcsin (1 / 2)$. Then

$$
\operatorname{Tr}(h)=0 .
$$

Proof. By the assumption (ex.48), there exists a selfadjoint element $b \in M_{n}(C([0,1])$ such that

$$
u(0) u(t)^{*}=\exp (\sqrt{-1} b(t)) \text { and }\|b\|<2 \arcsin (1 / 2) .
$$

However, $u(0) u(t)^{*} \in C U\left(M_{n}(C([0,1]))\right.$. It follows that

$$
\left(\frac{1}{2 \pi}\right) \operatorname{Tr}(b(t)) \in \mathbb{Z}
$$

Therefore $\operatorname{Tr}(b(t))$ is a constant. Since $b(0)=0$,

$$
\left(\frac{1}{2 \pi}\right) \operatorname{Tr}(b(t))=0 \text { for all } t \in[0,1] .
$$

Note that $h=b(1)$. Therefore

$$
\operatorname{Tr}(h)=0 .
$$

Theorem 3.6. Let $X$ be a compact metric space, let $\mathcal{F} \subset C(X)$ be a finite subset and let $\epsilon>0$ be a positive number. There exists $\eta_{1}>0$ satisfying the following: for any $\sigma_{1}>0$, there exists $\eta_{2}>0$ satisfying the following: for any $\sigma_{2}>0$, there exists $\eta_{3}>0$ satisfying the following: for any $\sigma_{3}>0$, there exists $\eta_{4}>0$ satisfying the following: For any $\sigma_{4}>0$, there exists $\gamma_{1}>0, \gamma_{2}>0, \delta>0$, a finite subset $\mathcal{G} \subset C(X)$ and a finite subset $\mathcal{P} \subset \underline{K}(C(X))$ a finite subset $\mathcal{H} \subset C(X)$ and a finite subset $\mathcal{U} \subset U_{c}\left(K_{1}(C(X))\right)$ for which $[\mathcal{U}] \subset \mathcal{P}$ satisfying the following: For any two unital $\delta$-G-multiplicative contractive completely positive linear maps $\varphi, \psi: C(X) \rightarrow M_{n}(C([0,1])$ such that

$$
\left.[\varphi]\right|_{\mathcal{P}}=\left.[\psi]\right|_{\mathcal{P}}=\left.[h]\right|_{\mathcal{P}}
$$

for some unital homomorphism $h: C(X) \rightarrow M_{n}(C([0,1]))$,

$$
\mu_{\tau \circ \varphi}\left(O_{r}\right) \geq \sigma_{i}, \quad \mu_{\tau \circ \psi}\left(O_{r}\right) \geq \sigma_{i},
$$

for all $\tau \in T\left(M_{n}(C([0,1]))\right)$ and for all $r \geq \eta_{i}, i=1,2,3$,

$$
\begin{array}{r}
|\tau \circ \varphi(g)-\tau \circ \psi(g)|<\gamma_{1} \text { for all } g \in \mathcal{H} \text { and } \\
\operatorname{dist}(\overline{\langle\varphi(u)\rangle}, \overline{\langle\psi(u)\rangle})<\gamma_{2} \text { for all } u \in \mathcal{U},
\end{array}
$$

there exists a unitary $W \in M_{n}(C([0,1]))$ such that

$$
\left\|W \varphi(f) W^{*}-\psi(f)\right\|<\epsilon \text { for all } f \in \mathcal{F} .
$$

(Note, as stated in 2.1, $\varphi$ and $\psi$ in (e3.51) are in fact $\varphi \otimes \operatorname{id}_{M_{k}}$ and $\psi \otimes \mathrm{id}_{m_{k}}$ for some integer $k \geq 1$. This will be used in the proof below.) 
Proof. Put $B=M_{n}(C([0,1]))$. We may write $C(X)=\lim _{n \rightarrow \infty}\left(C\left(Y_{n}\right), \imath_{n}\right)$, where $Y_{n}$ is a finite CW complex. Let $\epsilon>0$ and a finite subset $\mathcal{F} \subset C(X)$ be given. Without loss of generality, we may assume that $\mathcal{F} \subset \imath_{n}\left(C\left(Y_{n}\right)\right)$ for some $n$. Let $\eta_{1}^{\prime}>0$ (in place of $\eta$ ) be required by 2.14 for $\epsilon / 32$ (in place of $\epsilon$ ) and $\mathcal{F}$.

Let $\eta_{1}=\eta_{1}^{\prime} / 3$. Let $\sigma_{1}>0$ and let $\sigma_{1}^{\prime}=\sigma_{1} / 2>0$. Let $\delta_{1}>0$ (in place of $\epsilon$ ), $\mathcal{G}_{1} \subset C(X)$ (in place of $\mathcal{G}$ ) be a finite subset and let $\mathcal{P}_{0} \subset \underline{K}(C(X))$ (in place of $\mathcal{P}$ ) be a finite subset required by 2.14 for $\epsilon / 32$ (in place of $\epsilon$ ), $\mathcal{F}, \eta_{1}^{\prime}$ and $\sigma_{1}^{\prime}$. We may assume that $\delta_{1}<\epsilon / 32$.

There exists a finite $\mathrm{CW}$ complex $Y$, a unital homomorphism $\imath: C(Y) \rightarrow C(X)$ and a finite subset $\mathcal{F}^{\prime} \subset C(Y)$ such that $\imath\left(\mathcal{F}^{\prime}\right)=\mathcal{F}$ and $[\imath](\underline{K}(C(Y))) \supset \mathcal{P}_{0}$ (by choosing $Y=Y_{n}$ for some large $n$ ).

Let $0<\delta_{2}<\delta_{C(Y), \mathbf{b}}$ and $\mathcal{G}_{2}^{\prime} \supset \mathcal{F}_{C(Y), \mathbf{b}}$ such that $\left(\delta_{2}, \mathcal{G}_{2}^{\prime}\right)$ forms a $K K$-pair for $C(Y)$. Let $\mathcal{P}_{0}^{\prime} \subset \underline{K}(C(Y))$ be such that $\delta_{C(Y), \mathbf{b}}=\delta_{C, \mathcal{P}_{0}^{\prime}, \mathbf{b}}$. To simplify the notation, without loss of generality, we may assume that $[\imath]\left(\mathcal{P}_{0}^{\prime}\right)=\mathcal{P}_{0}$. Put $\mathcal{G}_{2}=\imath\left(\mathcal{G}_{2}^{\prime}\right)$.

Denote by $z \in C(\mathbb{T})$ the identity function on the unit circle. We may also assume that, for any $\delta_{2}-\{z, 1\} \times \mathcal{G}_{2}$-multiplicative contractive completely positive linear map $\Lambda: C(\mathbb{T}) \otimes C(Y) \rightarrow C$ (for any unital $C^{*}$-algebra $C$ with $T(C) \neq \emptyset$ ), $[\Lambda]$ is well defined and

$$
\tau([\Lambda(g)])=0
$$

for all $g \in \operatorname{Tor}\left(K_{1}(C(Y))\right)$ (which is a finite subgroup).

Furthermore, we may assume that $\delta_{2}$ is so small that if $\|u v-v u\|<3 \delta_{2}$, then the Exel formula

$$
\tau\left(\operatorname{bott}_{1}(u, v)\right)=\frac{1}{2 \pi \sqrt{-1}}\left(\tau\left(\log \left(u^{*} v u v^{*}\right)\right)\right.
$$

holds in any unital $C^{*}$-algebra $C$ with tracial rank zero and any $\tau \in T(C)$ (see Theorem 3.6 of [16]). Moreover if $\left\|v_{1}-v_{2}\right\|<3 \delta_{2}$, then

$$
\operatorname{bott}_{1}\left(u, v_{1}\right)=\operatorname{bott}_{1}\left(u, v_{2}\right) .
$$

Let $\mathcal{U}=\left\{g_{1}, g_{2}, \ldots, g_{k(X)}\right\} \subset U_{c}\left(K_{1}(C(X))\right.$ be a finite subset such that $\left\{\left[g_{1}\right],\left[g_{2}\right], \ldots,\left[g_{k(X)}\right]\right\}$ forms a set of generators for the finitely generated subgroup generated by $\mathcal{P}_{0} \cap K_{1}(C(X))$. We assume that $m(X) \geq 1$ is an integer and $g_{i} \in U\left(M_{m(X)}(C(X))\right.$. We may further assume that there are $g_{j}^{\prime}(j=1,2, \ldots, k(X))$ in $U_{c}\left(K_{1}(C(Y))\right)$ such that $\imath\left(g_{j}\right)=g_{j}^{\prime}, j=1,2, \ldots, k(X)$ (here again we identify a set of unitaries with its image in $U(C(Y)) / C U(C(Y))))$. Furthermore, we may assume that $g_{1}^{\prime}, g_{2}^{\prime}, \ldots, g_{k(X)}^{\prime}$ generate $K_{1}(C(Y))$. Let $\mathcal{U}_{0} \subset C(X)$ be a finite subset such that

$$
\mathcal{U}=\left\{\left(a_{i, j}\right): a_{i, j} \in \mathcal{U}_{0}\right\}
$$

Let $\delta_{u}=\min \left\{1 / 256 m(X)^{2}, \delta_{1} / 16 m(X)^{2}, \delta_{2} / 16 m(X)^{2}\right\}$ and $\mathcal{G}_{u}=\mathcal{F} \cup \mathcal{G}_{1} \cup \mathcal{G}_{2} \cup \mathcal{U}_{0}$.

Let $\eta_{2}^{\prime}>0$ (in place of $\eta$ ) and $\left\{s_{1}, s_{2}, \ldots, s_{k^{\prime}}\right\} \subset K_{1}(C(Y))$ be required by 3.1 for $\delta_{u}$ (in place of $\epsilon$ ) and $\mathcal{G}_{u}$ (in place of $\mathcal{F}$ ). Without loss of generality, we may assume that $\left\{s_{1}, s_{2}, \ldots, s_{k^{\prime}}\right\}=$ $\left\{g_{1}^{\prime}, g_{2}^{\prime}, \ldots, g_{k(X)}^{\prime}\right\}$ which generates $K_{1}(C(Y))$. Put $\eta_{2}=\eta_{2}^{\prime} / 3$.

Let $\sigma_{2}>0$ and let $\sigma_{2}^{\prime}=\sigma_{2} / 2$. Let $1>d>0$ be required by 3.1 for $\min \left\{\delta_{1} / 4, \delta_{2} / 4\right\}$ (in place $\epsilon), \mathcal{G}_{u}$ (in place of $\left.\mathcal{F}\right), \eta_{2}$ and $\sigma_{2}^{\prime}$.

Let $\delta_{3}>0$ (in place of $\delta$ ) and let $\mathcal{G}_{3} \subset C(\mathbb{T}) \otimes C(X)$ (in place of $\mathcal{G}$ ) be required by Lemma 10.3 of [21] for $d / 8$ (in place of $\sigma$ ) and $\mathbb{T} \times X$ (in place of $X$ ). Without loss of generality, we may assume that

$$
\mathcal{G}_{3}=\left\{z \otimes g: g \in \mathcal{G}_{4}^{\prime}\right\} \cup\left\{1 \otimes g: g \in \mathcal{G}_{4}\right\},
$$

where $\mathcal{G}_{4}^{\prime} \subset C(X)$ is a finite subset (by choosing a smaller $\delta_{3}$ and large $\mathcal{G}_{3}$ ).

Let $\epsilon_{1}^{\prime}>0$ (in place of $\delta$ ) and let $\mathcal{G}_{4}^{\prime \prime} \subset C(X)$ (in place of $\mathcal{G}$ ) be a finite subset required by 3.2 for $\eta_{1}, \eta_{2}, \sigma_{1}, \sigma_{2}, 1 / 2$ (in place of $\lambda_{1}$ ) and $1 / 4$ (in place of $\lambda_{2}$ ). 
Let $\epsilon_{1}^{\prime \prime}=\min \left\{d / 27 m(X)^{2}, \delta_{u} / 2, \delta_{3} / 2 m(X)^{2}, \epsilon_{1}^{\prime} / 2 m(X)^{2}\right\}$ and let $\bar{\epsilon}_{1}>0$ (in place of $\delta$ ) and $\mathcal{G}_{5} \subset C(X)$ (in place of $\mathcal{F}_{1}$ ) be a finite subset required by 2.8 of [17] for $\epsilon_{1}^{\prime \prime}$ (in place of $\epsilon$ ) and $\mathcal{G}_{u} \cup \mathcal{G}_{4}^{\prime} \cup \mathcal{G}_{4}^{\prime \prime}$ (and $C(X)$ in place of $B$ ). Put

$$
\epsilon_{1}=\min \left\{\epsilon_{1}^{\prime}, \epsilon_{1}^{\prime \prime}, \bar{\epsilon}_{1}\right\} .
$$

Let $\eta_{3}^{\prime}>0$ (in place of $\eta$ ) be required by 2.10 for $\epsilon_{1} / 4$ (in place $\epsilon$ ) and $\mathcal{G}_{5}$ (in place of $\mathcal{F}$ ).

Let $\eta_{3}^{\prime \prime}>0$ (in place of $\eta_{1}$ ) be required by 2.12 for $\epsilon_{1} / 4$ (in place of $\epsilon$ ) and $\mathcal{G}_{5}$ (in place of $\mathcal{F})$. Let $\eta_{3}=\min \left\{\eta_{3}^{\prime}, \eta_{3}^{\prime \prime}\right\}$. Let $\sigma_{3}>0$. Let $\gamma_{1}>0$ (in place of $\gamma$ ), $\delta_{4}>0, \mathcal{G}_{6} \subset C(X)$ (in place of $\mathcal{G}$ ), $\mathcal{H} \subset C(X)$ be a finite subset and let $\mathcal{P}_{1} \subset \underline{K}(C(X))$ (in place of $\mathcal{P}$ ) be required by 2.10 for $\epsilon_{1} / 4$ (in place of $\epsilon$ ), $\mathcal{G}_{5}$ (in place of $\mathcal{F}$ ), $\eta_{3}$ (in place $\eta$ ) and $\sigma_{3}$ (in place $\sigma$ ). Let $\eta_{4}>0$ (in place of $\eta_{2}$ ) be required by 2.12 for $\epsilon_{1} / 4$ (in place of $\epsilon$ ), $\mathcal{G}_{5}$ (in place of $\mathcal{F}$ ), $\eta_{3}$ (in place of $\eta_{1}$ ), $\sigma_{3}$ (in place of $\sigma_{1}$ ). Let $\sigma_{4}>0$. Let $\delta_{5}>0, \mathcal{G}_{7} \subset C(X)$ (in place of $\mathcal{G}$ ), $\mathcal{P}_{2} \subset \underline{K}(C(X)$ ) (in place of $\mathcal{P}$ ) be required by 2.12 ,

Let $\delta=\min \left\{\epsilon_{1} / 4, \delta_{4}, \delta_{5}\right\}, \mathcal{G}=\mathcal{G}_{5} \cup \mathcal{G}_{6} \cup \mathcal{G}_{7} \cup \mathcal{H}$ and $\mathcal{P}=\mathcal{P}_{0} \cup \mathcal{P}_{1} \cup \mathcal{P}_{2}$. Let $\gamma_{2}<$ $\min \left\{d / 16 m(X)^{2}, \delta_{u} / 9 m(X)^{2}, 1 / 256 m(X)^{2}\right\}$. We may assume that $(\delta, \mathcal{G}, \mathcal{P})$ is a $K L$-triple.

Now suppose that $\varphi, \psi: C(X) \rightarrow B$ are two unital $\delta$-G multiplicative contractive completely positive linear maps which satisfy the assumption for the above $\eta_{i}, \delta_{i}(i=1,2,3,4), \gamma_{i}(i=1,2)$, $\mathcal{P}, \mathcal{U}$ and $\mathcal{H}$.

Choose a partition

$$
0=t_{0}<t_{1}<\cdots<t_{N}=1
$$

such that

$$
\left\|\pi_{t} \circ \varphi(g)-\pi_{t_{i-1}} \circ \varphi(g)\right\|<\epsilon_{1} / 4 \text { and }\left\|\pi_{t} \circ \psi(g)-\pi_{t_{i-1}} \circ \psi(g)\right\|<\epsilon_{1} / 4
$$

for all $g \in \mathcal{G}$ and for all $t \in\left[t_{i-1}, t_{i}\right], i=1,2, \ldots, N$. By applying 2.10, for each $i$, there exists a unitary $w_{i} \in M_{n}$ such that

$$
\left\|w_{i} \pi_{t_{i}} \circ \varphi(g) w_{i}^{*}-\pi_{t_{i}} \circ \psi(g)\right\|<\epsilon_{1} / 4 \text { for all } g \in \mathcal{G}_{5}
$$

and, by 2.12, there are unital homomorphisms $h_{i, 1}, h_{i, 2}: C(X) \rightarrow M_{n}$ such that

$$
\left\|\pi_{t_{i}} \circ \varphi(g)-h_{i, 1}(g)\right\|<\epsilon_{1} / 4 \text { and }\left\|\pi_{t_{i}} \circ \psi(g)-h_{i, 2}(g)\right\|<\epsilon_{1} / 4 \text { for all } g \in \mathcal{G}_{5},
$$

$i=0,1,2, \ldots, N$. Moreover (by also applying 3.2 ),

$$
\mu_{t r \circ h_{i, j}}\left(O_{r}\right) \geq \sigma_{k}^{\prime}
$$

for all $r \geq \eta_{k}^{\prime}, k=1,2, j=1,2$ and $i=1,2, \ldots ., N$. Let $\omega_{j} \in M_{m(X)}(B)$ be a unitary such that $\omega_{j} \in C U\left(M_{m(X)}(B)\right.$ and

$$
\left\|\left\langle\varphi\left(g_{j}^{*}\right)\right\rangle\left\langle\psi\left(g_{j}\right)\right\rangle-\omega_{j}\right\|<\gamma_{2}, \quad i=1,2, \ldots, k(X) .
$$

Write

$$
\omega_{j}=\exp \left(\sqrt{-1} a_{j}\right)
$$

for some selfadjoint element $a_{j} \in M_{m(X)}\left(M_{n}(C([0,1]))\right), j=1,2, \ldots, k(X)$. Then

$$
\frac{n\left(t \otimes \operatorname{Tr}_{m(X)}\right)\left(a_{j}(s)\right)}{2 \pi} \in \mathbb{Z}
$$

$(s \in[0,1])$, where $t$ is the normalized trace on $M_{n}$. It follows that the above is a constant. In particular,

$$
n\left(t \otimes \operatorname{Tr}_{m(X)}\right)\left(a_{j}\left(t_{i}\right)\right)=n\left(t \otimes \operatorname{Tr}_{m(X)}\right)\left(a_{j}\left(t_{i-1}\right)\right)
$$


$i=1,2, \ldots, N$ and $j=1,2, \ldots, k(X)$.

Let $W_{i}=w_{i} \otimes \operatorname{id}_{M_{m(X)}}, i=0,1, \ldots, N$. Then

$\left\|\left(h_{i, 1} \otimes \operatorname{id}_{M_{m(X)}}\right)\left(g_{j}^{*}\right) W_{i}\left(h_{i, 1} \otimes \operatorname{id}_{M_{m(X)}}\right)\left(g_{j}\right) W_{i}^{*}-\omega_{j}\left(t_{i}\right)\right\|<3 m(X)^{2} \epsilon_{1}+2 \gamma_{2}<1 / 32(\mathrm{e} 3.60)$

It follows from (ex3.58) that there exists selfadjoint elements $b_{i, j} \in M_{n m(X)}$ such that

$$
\exp \left(\sqrt{-1} b_{i, j}\right)=\omega_{j}\left(t_{i}\right)^{*}\left(h_{i, 1} \otimes \operatorname{id}_{M_{m(X)}}\right)\left(g_{j}^{*}\right) W_{i}\left(h_{i, 1} \otimes \operatorname{id}_{M_{m(X)}}\right)\left(g_{j}\right) W_{i}^{*}
$$

such that

$$
\left\|b_{i, j}\right\|<2 \arcsin \left(3 m(X)^{2} \epsilon_{1} / 4+2 \gamma_{2}\right), \quad j=1,2, \ldots, k(X), i=0,1, \ldots, N .
$$

Note that

$$
\left(h_{i, 1} \otimes \operatorname{id}_{M_{m(X)}}\right)\left(g_{j}^{*}\right) W_{i}\left(h_{i, 1} \otimes \operatorname{id}_{M_{m(X)}}\right)\left(g_{j}\right) W_{i}^{*}=\omega_{j}\left(t_{i}\right) \exp \left(\sqrt{-1} b_{i, j}\right),
$$

$j=1,2, \ldots, k(X)$ and $i=0,1, \ldots, N$.

Then,

$$
\frac{n}{2 \pi}\left(t \otimes \operatorname{Tr}_{M_{m(X)}}\right)\left(b_{i, j}\right) \in \mathbb{Z},, \quad j=1,2, \ldots, k(X), i=0,1, \ldots, N
$$

Let

$$
\lambda_{i, j}=\frac{n}{2 \pi}\left(t \otimes \operatorname{Tr}_{\left.M_{m(X)}\right)}\right)\left(b_{i, j}\right)
$$

$j=0,1,2, \ldots, k(X), i=1,2, \ldots, N$. Note that $\lambda_{i, j} \in \mathbb{Z}$.

Define $\alpha_{i}^{(0,1)}: K_{1}(C(Y)) \rightarrow \mathbb{Z}$ by mapping $g_{j}^{\prime}$ to $\lambda_{i, j}, j=1,2, \ldots, m(X)$ and $i=0,1,2, \ldots, N$. We write $K_{0}(C(\mathbb{T}) \otimes C(Y))=K_{0}(C(Y)) \oplus \boldsymbol{\beta}\left(K_{1}(C(Y))\right)$ (see 2.10 of [17] for the definition of $\boldsymbol{\beta})$. Define $\alpha_{i}: K_{*}(C(\mathbb{T}) \otimes C(Y)) \rightarrow K_{*}\left(M_{n}\right)$ as follows

$$
\begin{array}{r}
\left.\alpha_{i}\right|_{K_{0}(C(\mathbb{T}) \otimes C(Y))}([1])=n, \\
\left.\alpha_{i}\right|_{\operatorname{ker} \rho_{C(Y)}}=0, \\
\left.\alpha_{i}\right|_{\boldsymbol{\beta}\left(K_{1}(C(Y))\right)}=\left.\alpha_{i} \circ \boldsymbol{\beta}\right|_{K_{1}(C(Y))}=\alpha_{i}^{(0,1)}, \\
\left.\alpha_{i}\right|_{K_{1}(C(\mathbb{T}) \otimes C(Y))}=0,
\end{array}
$$

By the Universal Coefficient Theorem $([26])$, there exists an element $\alpha_{i} \in K K(C(\mathbb{T}) \otimes C(Y), \mathbb{C})$ such that $\left.\alpha_{i}\right|_{K_{*}(C(\mathbb{T}) \otimes C(Y)}=\alpha_{i}$ as defined above, $i=1,2, \ldots, N$. We estimate that

$$
\left\|\left(w_{i-1}^{*} w_{i}\right) h_{i-1,1}(g)-h_{i-1,1}(g)\left(w_{i-1}^{*} w_{i}\right)\right\|<\epsilon_{1} \text { for all } g \in \mathcal{G}_{5} .
$$

Let $\Lambda_{i}: C(\mathbb{T}) \otimes C(X) \rightarrow M_{n}$ be a unital contractive completely positive linear map given by the pair $w_{i-1}^{*} w_{i}$ and $h_{i-1,1}, i=1,2, \ldots, N$ (see 2.8 of [17]). Denote $V_{i, j}=h_{i, 1} \otimes \operatorname{id}_{M_{m(X)}}\left(g_{j}\right)$, $j=1,2, \ldots, m(X)$ and $i=0,1,2, \ldots, N$. Note that

$$
\begin{aligned}
& \left\|W_{i-1} V_{i-1, j}^{*} W_{i-1}^{*} V_{i-1, j} V_{i-1, j}^{*} W_{i} V_{i-1, j} W_{i}^{*}-1\right\|<1 / 16 \\
& \left\|W_{i-1} V_{i-1, j}^{*} W_{i-1}^{*} V_{i-1, j} V_{i, j}^{*} W_{i} V_{i, j} W_{i}^{*}-1\right\|<1 / 16
\end{aligned}
$$

and there is a continuous path $Z(t)$ of unitaries such that $Z(0)=V_{i-1, j}$ and $Z(1)=V_{i, j}$. We obtain a continuous path

$$
W_{i-1} V_{i-1, j}^{*} W_{i-1}^{*} V_{i-1, j} Z(t)^{*} W_{i} Z(t) W_{i}^{*}
$$


which is in $C U\left(M_{n m(X)}\right)$ for all $t \in[0,1]$. It follows that

$$
(1 / 2 \pi \sqrt{-1})\left(t \otimes \operatorname{Tr}_{M_{m(X)}}\right)\left[\log \left(W_{i-1} V_{i-1, j}^{*} W_{i-1}^{*} V_{i-1, j} Z(t)^{*} W_{i} Z(t) W_{i}^{*}\right)\right]
$$

is a constant. In particular,

$$
\begin{array}{r}
(1 / 2 \pi \sqrt{-1})\left(t \otimes \operatorname{Tr}_{M_{m(X)}}\right)\left(\log \left(W_{i-1} V_{i-1, j}^{*} W_{i-1}^{*} W_{i} V_{i-1, j} W_{i}^{*}\right)\right) \\
=(1 / 2 \pi \sqrt{-1})\left(t \otimes \operatorname{Tr}_{M_{m(X)}}\right)\left(\log \left(W_{i-1} V_{i-1, j}^{*} W_{i-1}^{*} V_{i-1, j} V_{i, j}^{*} W_{i} V_{i, j} W_{i}^{*}\right)\right) .
\end{array}
$$

Also

$$
\begin{aligned}
& W_{i-1} V_{i-1, j}^{*} W_{i-1}^{*} V_{i-1, j} V_{i, j}^{*} W_{i} V_{i, j} W_{i}^{*} \\
& =\left(\omega_{j}\left(t_{i-1}\right) \exp \left(\sqrt{-1} b_{i-1, j}\right)\right)^{*} \omega_{j}\left(t_{i}\right) \exp \left(\sqrt{-1} b_{i, j}\right) \\
& =\exp \left(-\sqrt{-1} b_{i-1, j}\right) \omega_{j}\left(t_{i-1}\right)^{*} \omega_{j}\left(t_{i}\right) \exp \left(\sqrt{-1} b_{i j}\right) .
\end{aligned}
$$

Note that, by (e3.58) and (e3.60),

$$
\left\|\omega_{j}\left(t_{i-1}\right)^{*} \omega_{j}\left(t_{i}\right)-1\right\|<3\left(3 \epsilon_{1}^{\prime}+2 \gamma_{2}\right)<3 / 32,
$$

$j=1,2, \ldots, m(X), i=1,2, \ldots, N$. By 3.5

$$
\left(t \otimes \operatorname{Tr}_{m(X)}\right)\left(\log \left(\omega_{j}\left(t_{i-1}\right)^{*} \omega_{j}\left(t_{i}\right)\right)\right)=0 .
$$

It follows that (by the Exel formula, using (e3.73), (e3.76) and (e3.78)

$$
\begin{aligned}
(t & \left.\otimes \operatorname{Tr}_{m(X)}\right)\left(\operatorname{bott}\left(V_{i-1, j}, W_{i-1}^{*} W_{i}\right)\right) \\
& =\left(\frac{1}{2 \pi \sqrt{-1}}\right)\left(t \otimes \operatorname{Tr}_{m(X)}\right)\left(\log \left(V_{i-1, j}^{*} W_{i-1}^{*} W_{i} V_{i-1, j} W_{i}^{*} W_{i-1}\right)\right) \\
& =\left(\frac{1}{2 \pi \sqrt{-1}}\right)\left(t \otimes \operatorname{Tr}_{m(X)}\right)\left(\log \left(W_{i-1} V_{i-1, j}^{*} W_{i-1}^{*} W_{i} V_{i-1, j} W_{i}^{*}\right)\right) \\
& =\left(\frac{1}{2 \pi \sqrt{-1}}\right)\left(t \otimes \operatorname{Tr}_{m(X)}\right)\left(\log \left(W_{i-1} V_{i-1, j}^{*} W_{i-1}^{*} V_{i-1, j} V_{i, j}^{*} W_{i} V_{i, j} W_{i}^{*}\right)\right) \\
& =\left(\frac{1}{2 \pi \sqrt{-1}}\right)\left(t \otimes \operatorname{Tr}_{m(X)}\right)\left(\log \left(\exp \left(-\sqrt{-1} b_{i-1, j}\right) \omega_{j}\left(t_{i-1}\right)^{*} \omega_{j}\left(t_{i}\right) \exp \left(\sqrt{-1} b_{i j}\right)\right)\right. \\
& =\left(\frac{1}{2 \pi \sqrt{-1}}\right)\left[\left(t \otimes \operatorname{Tr}_{k(n)}\right)\left(-\sqrt{-1} b_{i-1, j}\right)+\left(t \otimes \operatorname{Tr}_{k(n)}\right)\left(\log \left(\omega_{j}\left(t_{i-1}\right)^{*} \omega_{j}\left(t_{i}\right)\right)\right.\right. \\
& \left.\quad+\left(t \otimes \operatorname{Tr}_{k(n)}\right)\left(\sqrt{-1} b_{i, j}\right)\right] \\
& =\frac{1}{2 \pi}\left(t \otimes \operatorname{Tr}_{k(n)}\right)\left(-b_{i-1, j}+b_{i, j}\right) .
\end{aligned}
$$

In other words,

$$
\left.\operatorname{bott}\left(V_{i-1, j}, W_{i-1}^{*} W_{i}\right)\right)=-\lambda_{i-1, j}+\lambda_{i, j}
$$

$j=1,2, \ldots, m(X)$ and $i=1,2, \ldots, N$.

Define $\beta_{0}=0, \beta_{1}=\left[\Lambda_{1}\right]-\alpha_{1}+\alpha_{0}+\beta_{0}$ and

$$
\beta_{i}=\left[\Lambda_{i}\right]-\alpha_{i}+\alpha_{i-1}+\beta_{i-1}, \quad i=1,2, \ldots, N .
$$

Define $\kappa_{0}=0$ and $\kappa_{i}=\alpha_{i}+\beta_{i}, i=1,2, \ldots, N$. Note that $\left.\alpha_{i}, \beta_{i}, \kappa_{i} \in K K(C(\mathbb{T}) \otimes C(Y)), \mathbb{C}\right)$. We compute that

$$
\begin{aligned}
& \beta_{1}\left(g_{j}^{\prime}\right)=\left[\Lambda_{1}\right]\left(g_{j}^{\prime}\right)-\lambda_{1, j}+\lambda_{0, j}=0 \\
& \beta_{2}\left(g_{j}^{\prime}\right)=\left[\Lambda_{2}\right]\left(g_{j}^{\prime}\right)-\lambda_{2, j}+\lambda_{1, j}+\beta_{1}\left(g_{j}^{\prime}\right)=0 \text { and } \\
& \beta_{i}\left(g_{j}^{\prime}\right)=0, \quad i=1,2, \ldots, N \text { and } j=1,2, \ldots, k(X) .
\end{aligned}
$$


It follows that

$$
\left|\tau \otimes \operatorname{Tr}_{m(X)}\left(\kappa_{i}\left(\left[g_{i}\right]\right)\right)\right|=\left|\lambda_{i, j} / n\right|<d / 2,
$$

$j=1,2, \ldots, N$ and $i=1,2, \ldots, k(X)$. By applying [3.1, there is, for each $i=1,2, \ldots, N$, a unitary $z_{i} \in M_{n}$ such that

$$
\begin{array}{r}
\left\|\left[z_{i}, h_{i, 1}(g)\right]\right\|<\delta_{u} \text { for all } g \in \mathcal{G}_{u} \\
\text { and } \operatorname{Bott}\left(z_{i}, h_{i, 1} \circ \imath\right)=\kappa_{i}, \quad i=0,1,2, \ldots, N-1 \text {. }
\end{array}
$$

Let $U_{i}=z_{i-1} w_{i-1}^{*} w_{i} z_{i}^{*}, i=1,2, \ldots, N$. Then

$$
\left\|\left[U_{i}, h_{i-1,1}(g)\right]\right\|<\min \left\{\delta_{1}, \delta_{2}\right\} \text { for all } g \in \mathcal{G}_{u},
$$

$i=1,2, \ldots, N$. Moreover

$$
\begin{aligned}
\operatorname{Bott}\left(U_{i}, h_{i-1,1} \circ \imath\right)= & \operatorname{Bott}\left(z_{i, 1}, h_{i-1,1} \circ \imath\right)+\operatorname{Bott}\left(w_{i-1}^{*} w_{i}, h_{i-1,1} \circ \imath\right) \\
& +\operatorname{Bott}\left(z_{i}^{*}, h_{i-1,1} \circ \imath\right) \\
= & \kappa_{i-1}+\left[\Lambda_{i}\right]-\kappa_{i} \\
= & \alpha_{i-1}+\beta_{i-1}+\left[\Lambda_{i}\right]-\alpha_{i}-\beta_{i} \\
= & \alpha_{i-1}+\beta_{i-1}+\left[\Lambda_{i}\right]-\alpha_{i}-\left(\left[\Lambda_{i}\right]-\alpha_{i}+\alpha_{i-1}+\beta_{i-1}\right) \\
= & 0
\end{aligned}
$$

$i=0,1,2, \ldots, N-1$. It follows that

$$
\left.\operatorname{Bott}\left(U_{i}, h_{i-1,1}\right)\right|_{\mathcal{P}}=0, \quad i=1,2, \ldots, N-1 .
$$

By applying 2.14, there exists a continuous path of unitaries, $\left\{U_{i}(t): t \in\left[t_{i-1}, t_{i}\right]\right\}$ such that

$$
\begin{gathered}
U_{i}\left(t_{i-1}\right)=1, \quad U_{i}\left(t_{i}\right)=z_{i-1} w_{i-1}^{*} w_{i} z_{i}^{*} \text { and } \\
\left\|U(t) h_{i-1,1}(f) U(t)^{*}-h_{i-1,1}(f)\right\|<\epsilon / 32
\end{gathered}
$$

for all $f \in \mathcal{F}$ and for all $t \in\left[t_{i-1}, t_{i}\right], i=1,2, \ldots, N$. Define $W \in B$ by

$$
W(t)=w_{i-1} z_{i-1}^{*} U_{i}(t) \text { for all } t \in\left[t_{i-1}, t_{i}\right], \quad i=1,2, \ldots, N .
$$

Note that $W\left(t_{i-1}\right)=w_{i-1} z_{i-1}^{*}, i=1,2, \ldots, N$, and $W(1)=w_{N} z_{N}^{*}$. One checks that, by (e 3.56), (e3.102), (e 3.93), for $t \in\left[t_{i-1}, t_{i}\right]$,

$$
\begin{aligned}
& \left\|W(t) \pi_{t} \circ \varphi(f) W(t)^{*}-\pi_{t} \circ \psi(f)\right\| \\
& <\left\|W(t) \pi_{t} \circ \varphi(f) W(t)^{*}-W(t) \pi_{t_{i-1}} \circ \varphi(f) W(t)^{*}\right\| \\
& +\left\|W(t) \pi_{t_{i-1}} \circ \varphi(f) W(t)^{*}-W(t) h_{i-1,1}(f) W(t)^{*}\right\| \\
& +\left\|W(t) h_{i-1,1}(f) W(t)^{*}-W\left(t_{i-1}\right) h_{i-1,1}(f) W\left(t_{i-1}\right)^{*}\right\| \\
& +\left\|W\left(t_{i-1}\right) h_{i-1,1}(f) W\left(t_{i-1}\right)^{*}-w_{i-1} h_{i-1,1}(f) w_{i-1}^{*}\right\| \\
& +\left\|w_{i-1} h_{i-1,1}(f) w_{i-1}^{*}-w_{i-1} \pi_{t_{i-1}} \circ \varphi(f) w_{i-1}^{*}\right\| \\
& +\left\|w_{i-1} \pi_{t_{i-1}} \circ \varphi(f) w_{i-1}^{*}-\pi_{t_{i-1}} \circ \psi(f)\right\| \\
& +\left\|\pi_{t_{i-1}} \circ \psi(f)-\pi_{t} \circ \psi(f)\right\| \\
& <\epsilon_{1} / 4+\epsilon_{1} / 4+\epsilon / 32+\delta_{u}+\epsilon_{1} / 4+\epsilon_{1} / 4+\epsilon_{1} / 4<\epsilon
\end{aligned}
$$

for all $f \in \mathcal{F}$.

Remark 3.7. By an argument used in 5.1, we can remove the part of the assumption in (e3.49) that $\left.[\varphi]\right|_{\mathcal{P}}$ is the same as $\left.[h]\right|_{\mathcal{P}}$ for some homomorphism. At present, we do not use that form of the statement. 


\section{Preparation for the proof}

Lemma 4.1. There is an integer $K>0$ satisfying the following condition: Suppose that $u \in$ $M_{n}\left(C([0,1])\right.$ for some integer $n \geq K$. Then, for any integer $k>0$ and any $L>0$, if $\operatorname{cel}\left(u^{k}\right) \leq L$, then $\operatorname{cel}(u) \leq 2 \pi / K+L / k+6 \pi$.

Proof. (See the proof of 6.10 of [15].) Let $K=K(1)$ be given in Lemma 3.4 of [25]. Let $n \geq K$. It follows from Lemma 3.3 (1) of [25] that there exists a selfadjoint element $a \in M_{n}(C([0,1]))$ with $\|a\| \leq L$ such that

$$
\operatorname{det}\left(\exp (i a) u^{k}\right)(t)=1
$$

for every $t \in[0,1]$. Fix one of such integer $n$. So

$$
\operatorname{det}\left((\exp (i a / k) u)^{k}\right)(t)=1
$$

for all $t \in[0,1]$. This implies that, for each $t \in[0,1]$,

$$
\operatorname{det}(\exp (i a(t) / k) u(t))=\exp (2 \pi i l(t) / k)
$$

for some integer $l(t) \leq k$. Suppose that $b(t)=-2 \pi l(t) / k$. Then $b(t)$ is a real valued continuous function on $[0,1]$, whence it is a constant. Note that

$$
\exp (i(b(t) / n) \exp (i a / k)=\exp (i(b(t) / n+a / k))
$$

Then

$$
\operatorname{det}(\exp (i(b(t) / n) \exp (i a / k) u)=1
$$

(for all $t \in[0,1]$ ). By 3.4 and 3.1 of [25],

$$
\operatorname{cel}(u) \leq 2 \pi / K+L / k+6 \pi
$$

Theorem 4.2. Let $X$ be a compact metric space. Let $\epsilon>0$ and let $\mathcal{F} \subset C(X)$ be a finite subset. Suppose that $\lambda: U_{c}\left(K_{1}(C(X))\right) \rightarrow \mathbb{R}_{+}$is a map. There exist $\delta>0$, a finite subset $\mathcal{G} \subset C(X)$, a finite subset $\mathcal{P} \subset \underline{K}(C(X))$, a finite subset of unitaries $\mathcal{U} \subset U_{c}\left(K_{1}(C(X))\right)$ and an integer $L>0$ satisfying the following condition: if $\varphi, \psi: C(X) \rightarrow C\left([0,1], M_{n}\right)$ (for some integer $n \geq 1$ ) are two unital $\delta-\mathcal{G}$-multiplicative contractive completely positive linear maps such that

$$
\left.[\varphi]\right|_{\mathcal{P}}=\left.[\psi]\right|_{\mathcal{P}} \text { and } \operatorname{dist}(\overline{\langle\varphi(u)\rangle}, \overline{\langle\psi(u)\rangle}) \leq \lambda(u)
$$

for all $u \in \mathcal{U}$, then there is a homomorphism $\Phi: C(X) \rightarrow M_{L}\left(M_{n}(C([0,1]))\right)$ with finite dimensional range and a unitary $U \in M_{L+1}\left(M_{n}\left(C\left([0,1], M_{n}\right)\right)\right)$ such that

$$
\left\|U^{*} \operatorname{diag}(\varphi(f), \Phi(f)) U-\operatorname{diag}(\psi(f), \Phi(f))\right\|<\epsilon
$$

for all $f \in \mathcal{F}$.

Proof. This follows from Theorem 3.2 of [7]. One takes $B=M_{n}(C([0,1]))$. Note that $B$ has stable rank one and $K_{0}$-divisible rank $T$, where $T: N \times N$ is defined by $T(k, m)=[m / k]+1$. Let $K$ be the constant described in Lemma 3.4 of [25] (for $d=1$ ). Pick a point $\xi \in X$. If $n \geq K$, we continue the argument below. If $n<K$, define $\varphi_{0}: C(X) \rightarrow M_{K-n}(C([0,1]))$ by $\varphi_{0}(f)=f(\xi) \operatorname{id}_{M_{K-n}}$ for all $f \in C(X)$. Replacing $\varphi$ and $\psi$ by $\varphi \oplus \varphi_{0}$ and $\psi \oplus \varphi_{0}$ and late absorbing $\varphi_{0}$, we see that we may assume that $n \geq K$. 
Then, by 4.1, $B$ has exponential divisible rank $E(L, k)$, where $E(l, k) \leq 2 \pi / K+L / k+6 \pi$. It is also easy to see that $\operatorname{cer}(B) \leq 2$. Then define $\Lambda: U\left(M_{\infty}(C(X)) \rightarrow \mathbb{R}_{+}\right.$as follows:

Let $\Pi: U\left(M_{\infty}(C(X))\right) \rightarrow K_{1}(C(X))$ be the quotient map and let $J: K_{1}(C(X)) \rightarrow$ $\cup_{n=1}^{\infty} U\left(M_{n}(C(X))\right) / C U(C(X))$ be the splitting map (see 2.4). If $v \in U\left(M_{n}(C(X))\right)$ and $\Pi(v) \neq$ 0 , define $v_{0}=v\left(J \circ \Pi\left(v^{*}\right)\right)_{c}$, where $J \circ \Pi\left(v^{*}\right)_{c} \in U_{c}\left(K_{1}(C(X))\right)$. Define $\Lambda: U\left(M_{\infty}(C(X))\right) \rightarrow \mathbb{R}_{+}$ as follows:

$$
\begin{aligned}
& \Lambda(v)=2 \operatorname{cel}(v)+1 \text { if } v \in \cup_{n=1}^{\infty} U_{0}\left(M_{n}(C(X))\right) \text { and } \\
& \Lambda(v)=\lambda\left(J \circ \Pi(v)_{c}\right)+6 \pi+2 \operatorname{cel}\left(v_{0}\right)+1 \text { if } \Pi(v) \neq 0
\end{aligned}
$$

where $\operatorname{cel}(v)$ and $\operatorname{cel}\left(v_{0}\right)$ is the exponential length of $v$ and $v_{0}$ in $\cup_{n=1}^{\infty} U_{0}\left(M_{n}(C(X))\right)$, respectively.

Note that, for any finite subset $\mathcal{V} \subset U\left(M_{m}(C(X))\right.$ ) (for some integer $m \geq 1$ ), if $\delta$ is sufficiently small and $\mathcal{G}$ is sufficiently large (depends only on $\mathcal{V}$ ),

$$
\operatorname{cel}\left(\varphi(v) \psi(v)^{*}\right) \leq 2 \operatorname{cel}(v)+1 / 4 \leq \Lambda(v) \text { for all } v \in \mathcal{V} \text { and } \Pi(v)=0 .
$$

Otherwise, if $\Pi(v) \neq 0, v=v_{c} v_{0}$ for some $v_{c} \in U_{c}\left(K_{1}(C(X))\right)$ and $v_{0} \in U_{0}\left(M_{\infty}(C(X))\right)$. Thus, if $v_{c} \in \mathcal{U}, \delta$ is sufficiently small and $\mathcal{G}$ is sufficiently large (depends only on $\mathcal{V}$ and $\mathcal{U}$ ),

$$
\begin{aligned}
\operatorname{cel}\left(\varphi(v) \psi\left(v^{*}\right)\right) & =\operatorname{cel}\left(\varphi\left(v_{c} v_{0}\right) \psi\left(v_{0}^{*} v_{c}^{*}\right)\right) \\
& \leq \operatorname{cel}\left(\varphi(v) \psi(v)^{*}\right)+1 / 4+\operatorname{cel}\left(\varphi\left(v_{c}\right) \psi\left(v_{c}^{*}\right)\right. \\
& \leq 2 \operatorname{cel}(v)+1 / 4+1 / 4+\lambda\left(v_{c}\right)+6 \pi \leq \Lambda(v) .
\end{aligned}
$$

Therefore we can apply Theorem 3.2 of [7] directly (and the point-evaluation $f \mapsto f(\xi) \operatorname{id}_{M_{k}}$ will be absorbed into $\Phi)$.

The following is a folklore. It is a special case of Theorem 3.2 of [7]. It also follows from 2.12. We state here for the convenience for our proofs.

Lemma 4.3. Let $X$ be a compact metric space, let $\epsilon>0$ and let $\mathcal{F} \subset C(X)$ be a finite subset. There exists $\delta>0$, a finite subset $\mathcal{G} \subset C(X)$ and a finite subset $\mathcal{P} \subset \underline{K}(C(X))$ which forms a KL-triple for $C(X)$ and an integer $N$ satisfying the following: Suppose that $\varphi: C(X) \rightarrow F$ is a unital $\delta$-G-multiplicative contractive completely positive linear map, where $F$ is a finite dimensional $C^{*}$-algebra such that

$$
\left.[\varphi]\right|_{\mathcal{P}}=\left.[H]\right|_{\mathcal{P}}
$$

for some unital homomorphism $H: C(X) \rightarrow F$. Then there exists a unital homomorphism $\Phi: C(X) \rightarrow M_{N}(F)$ and a unital homomorphism $h: C(X) \rightarrow M_{N+1}(F)$ such that

$$
\|\varphi(f) \oplus \Phi(f)-h(f)\|<\epsilon
$$

for all $f \in \mathcal{F}$.

The following is an easy folklore.

Lemma 4.4. Let $X$ be a compact metric space, $\epsilon>0$ be a positive number and let $\mathcal{F} \subset C(X)$ be a finite subset. Then, there exists a finite subset $\left\{x_{1}, x_{2}, \ldots, x_{m}\right\} \subset X$ satisfying the following: For any unital homomorphism $\varphi: C(X) \rightarrow A$ (for any unital $C^{*}$-algebra $A$ ) with finite dimensional range and any $K \geq m$, there is a unital homomorphism $\psi: C(X) \rightarrow M_{K}$ with finite dimensional range and a unitary $u \in M_{K+1}(A)$ such that

$$
\left\|u^{*}(\varphi(f) \oplus \psi(f)) u-H(f)\right\|<\epsilon \text { for all } f \in \mathcal{F},
$$

where $H(f)=\sum_{i=1}^{m} f\left(x_{i}\right) p_{i}$ for all $f \in C(X)$ and where $p_{1}, p_{2}, \ldots, p_{m} \in M_{k+1}(A)$ is a set of mutually orthogonal projections such that $1_{A} \lesssim p_{i}$ (for all $i$ ) and $\sum_{i=1}^{m} p_{i}=1_{M_{K+1}}$. 
Proof. Choose $\eta>0$ such that

$$
\left|f(x)-f\left(x^{\prime}\right)\right|<\epsilon / 2 \text { for all } f \in \mathcal{F},
$$

provided that $\operatorname{dist}\left(x, x^{\prime}\right)<2 \eta$. Choose a finite $\eta / 2$ net $\left\{x_{1}, x_{2}, \ldots, x_{m}\right\}$ of $X$. Write $\varphi(f)=$ $\sum_{i=1}^{n} f\left(y_{i}\right) e_{i}$ for all $f \in C(X)$, where $\left\{y_{1}, y_{2}, \ldots, y_{n}\right\} \subset X$ and $\left\{e_{1}, e_{2}, \ldots, e_{n}\right\} \subset A$ is a set of mutually orthogonal projections. Let $Y_{1}, Y_{2}, \ldots, Y_{m}$ be mutually disjoint subsets of $\left\{y_{1}, y_{2}, \ldots, y_{n}\right\}$ such that $Y_{j} \in O\left(x_{j}, \eta\right), j=1,2, \ldots, m$. Note that $Y_{j}$ could be empty. We rewrite

$$
\varphi(f)=\sum_{i=1}^{m}\left(\sum_{y_{j} \in Y_{i}} f\left(y_{j}\right) e_{j}\right)
$$

Denote $E_{i}=\sum_{y_{j} \in Y_{i}} e_{j}$. Note that if $Y_{i}=\emptyset, E_{i}=0$. Now let $K \geq m$. Choose mutually orthogonal $p_{1}^{\prime}, p_{2}^{\prime}, \ldots, p_{K+1}^{\prime}$ in $M_{K+1}(A)$ such that each $p_{i}$ is unitarily equivalent to $1_{A}$. Let $E_{i}^{\prime} \leq p_{i}^{\prime}$ be such that $E_{i}^{\prime}$ is unitarily equivalent to $E_{i}, i=1,2, \ldots, m$. Define $p_{i}=p_{i}^{\prime}, i=1,2, \ldots, m-1$ and $p_{m}=\sum_{j=m}^{K+1} p_{j}^{\prime}$ and define $q_{i}=p_{i}-E_{i}^{\prime}, i=1,2, \ldots, m$. Define

$$
\begin{array}{r}
\psi(f)=\sum_{i=1}^{m} f\left(x_{i}\right) q_{i} \text { and } \\
H(f)=\sum_{i=1}^{m} f\left(x_{i}\right) p_{i}
\end{array}
$$

One then ready to verify that $\psi$ and $H$ meet the requirements.

Added in proof: The author would like to thank Junping Liu who brought the issue of the above lemma. He also benefited from a conversation with Guihua Gong about the same issue.

Lemma 4.5. Let $X$ be a compact metric space. Let $\lambda: \bigcup_{n=1}^{\infty} U\left(M_{n}(C(X))\right) \rightarrow \mathbb{R}_{+}$be a map. For any $\epsilon>0$ and any finite subset $\mathcal{F} \subset C(X)$, there exist $\delta>0$ a finite subset $\mathcal{G} \subset C(X)$, a finite subset $\mathcal{P} \subset \underline{K}(C(X))$ and a finite subset of unitaries $\mathcal{U} \subset U_{c}(C(X))$, a finite subset $\left\{x_{1}, x_{2}, \ldots, x_{m}\right\} \subset X$ and an integer $L>0$ satisfying the following condition: if $\varphi, \psi: C(X) \rightarrow A$ (for any unital separable simple $C^{*}$-algebra $A$ with tracial rank at most one) are unital $\delta$ - $\mathcal{G}$ multiplicative contractive completely positive linear maps such that

$$
\begin{array}{r}
{\left.[\psi]\right|_{\mathcal{P}}=\left.[\varphi]\right|_{\mathcal{P}} \text { and }} \\
\operatorname{dist}(\langle\varphi(u)\rangle,\langle\psi(u)\rangle) \leq \lambda(u)
\end{array}
$$

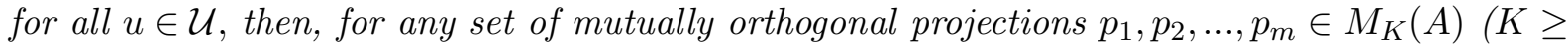
$m L)$ with $\left[p_{i}\right] \geq L\left[1_{A}\right], i=1,2, \ldots, m$, and $\sum_{i=1}^{m} p_{i}=1_{M_{K}(A)}$, there is a unitary $U \in M_{K+1}(A)$ such that

$$
\left\|U^{*} \operatorname{diag}(\varphi(f), H(f)) U-\operatorname{diag}(\psi(f), H(f))\right\|<\epsilon
$$

for all $f \in \mathcal{F}$, where $H(f)=\sum_{i=1}^{m} f\left(x_{i}\right) p_{i}$ for all $f \in C(X)$.

Proof. The proof follows exactly the same way as that of 4.2. Note that it follows from [19] that $M_{j}(A)$ has exponential rank $1+\epsilon$ for every integer $j \geq 1$. Also, by [15], $A$ has stable rank one, $K_{0}$-divisible rank one, exponential divisible $\operatorname{rank} E(L, k)=L / k+8 \pi+1$ (see 6.10 of [15], or derive it from 4.1 directly). Thus Theorem 3.2 of [7] (together with 4.4) can also be applied as in the proof of 4.2 . 
Lemma 4.6. Let $X$ be a compact metric space and let $s_{1}, s_{2}, \ldots, s_{m} \in \operatorname{ker} \rho_{C(X)}$ be a finite subset. For any $d>0$, there is $\delta>0$ and $\mathcal{G} \subset C(X)$ satisfying the following: For any unital $C^{*}$-algebra $A$ with $T(A) \neq \emptyset$ and any unital $\delta$-G-multiplicative contractive completely positive linear map $L: C(X) \rightarrow A$, one has that

$$
\tau\left([L]\left(s_{j}\right)\right)<d \text { for all } \tau \in T(A), \quad j=1,2, \ldots, m .
$$

Proof. There is an integer $m_{0} \geq 1$ and projections $p_{i}, q_{i} \in M_{m_{0}}(C(X))$ such that

$$
\left[p_{i}\right]-\left[q_{i}\right]=s_{i}, \quad i=1,2, \ldots, m .
$$

Note that, for any $\tau \in T(A)$,

$$
\tau\left(p_{i}\right)=\tau\left(q_{i}\right), \quad i=1,2, \ldots, m .
$$

Now suppose the lemma is false. Then there is $d_{0}>0$, a sequence of unital $C^{*}$-algebras $A_{n}$ with $T\left(A_{n}\right) \neq \emptyset$, a sequence of $\delta_{n}-\mathcal{G}_{n}$-multiplicative contractive completely positive linear maps $L_{n}: C(X) \rightarrow A_{n}$ with $\sum_{n=1}^{\infty} \delta_{n}<\infty$ and $\cup_{n=1}^{\infty} \mathcal{G}_{n}$ is dense in $C(X)$ such that, for some $\tau_{n} \in T\left(A_{n}\right)$ and $1 \leq j \leq m$,

$$
\left.\mid \tau_{n}\left(L_{n} \otimes \operatorname{id}_{M_{m_{0}}}\right)\left(p_{j}-q_{j}\right)\right) \mid \geq d_{0}
$$

for all $n$. Define $L: C(X) \rightarrow \prod_{n=1}^{\infty} A_{n}$ by $L(f)=\left\{L_{n}(f)\right\}$ for all $f \in C(X)$. Let $\pi: \prod_{n=1}^{\infty} A_{n} \rightarrow$ $\prod_{n=1}^{\infty} A_{n} / \oplus_{n=1}^{\infty} A_{n}$ be the quotient map. Then $\pi \circ L: C(X) \rightarrow \prod_{n=1}^{\infty} A_{n} / \oplus_{n=1}^{\infty} A_{n}$ is a unital homomorphism. Therefore, for any tracial state $t \in T\left(\prod_{n=1}^{\infty} A_{n} / \oplus_{n=1}^{\infty} A_{n}\right)$,

$$
\left.t\left((\pi \circ L) \otimes \operatorname{id}_{M_{m_{0}}}\right)\left(p_{j}-q_{j}\right)\right)=0 .
$$

Let $T_{n}: \prod_{n=1}^{\infty} A_{n} \rightarrow \mathbb{C}$ be defined by $T_{n}(a)=\tau_{n}\left(\pi_{n}(a)\right)$ for all $a \in \prod_{n=1}^{\infty} A_{n}$, where $\pi_{n}$ : $\prod_{n=1}^{\infty} A_{n} \rightarrow A_{n}$ is the projection to the $n$-coordinate. Then $T_{n}$ is a tracial state. Note that, for any $a \in \oplus_{n=1}^{\infty} A_{n}$,

$$
\lim _{n \rightarrow \infty} T_{n}(a)=0 .
$$

Let $T$ be a limit point of $\left\{T_{n}\right\}$. Then, by (e 4.134), $T$ defines a tracial state on $\prod_{n=1}^{\infty} A_{n} / \oplus_{n=1}^{\infty} A_{n}$. Therefore, by (e4.133),

$$
T\left(\left(\left(\pi \circ L_{* 0}\right) \otimes \operatorname{id}_{M_{m_{0}}}\right)\left(p_{j}-q_{j}\right)\right)=0 .
$$

It then follows that, for some subsequence $\left\{n_{k}\right\}$,

$$
\lim _{k \rightarrow \infty} \tau_{n_{k}}\left(\left(L_{n} \otimes \operatorname{id}_{M_{m_{0}}}\right)\left(p_{j}-q_{j}\right)\right)=0 .
$$

This contradicts with (e4.132). The lemma follows.

When $K_{i}(C(X))(i=0,1)$ is finitely generated, the following follows from 10.2 of [21]. We make a modification so it also applies to the case that $K_{i}(C(X))(i=0,1)$ is not finitely generated.

Lemma 4.7. Let $X$ be a compact metric space. For any $\delta>0$, any finite subset $\mathcal{G} \subset C(X)$ and any finite subset $\mathcal{P} \subset \underline{K}(C(X))$ for which the intersection of $\operatorname{ker} \rho_{C(X)}$ and the subgroup generated by $\mathcal{P}$ is generated by $g_{1}, g_{2}, \ldots, g_{k}$ such that $(\delta, \mathcal{G}, \mathcal{P})$ is a $K L$-triple, there exists an integer $N(\delta, \mathcal{G}, \mathcal{P})$ satisfies the following: 
For any unital $\delta$-G-multiplicative contractive completely positive linear map $L: C(X) \rightarrow$ $B$, where $B=M_{n}$, or $B=M_{n}\left(C([0,1])\right.$ ) (for any integer $n \geq 1$ ) with $K=\max \left\{\left|L\left(g_{i}\right)\right|\right.$ : $i=1,2, \ldots, k\}$, There exists an integer $N(K) \geq 1$ satisfying the following: for any integer $N \geq N(K) / n$, there exists a unital $\delta$-G-multiplicative contractive completely positive linear map $L_{0}: C(X) \rightarrow M_{n N} \subset M_{N}(B) \subset$ such that

$$
\begin{aligned}
\frac{N(K)}{\max \{K, 1\}} & \leq N(\delta, \mathcal{G}, \mathcal{P}) \text { and } \\
\left.\left([L]+\left[L_{0}\right]\right)\right|_{\mathcal{P}} & =\left.[H]\right|_{\mathcal{P}}
\end{aligned}
$$

for some unital homomorphism $H: C(X) \rightarrow M_{1+N}(B)$ with finite dimensional range.

Proof. Write $C(X)=\lim _{n \rightarrow \infty} C\left(Y_{n}\right)$, where each $Y_{n}$ is a finite CW complex. We use $\imath_{m}$ : $C\left(Y_{m}\right) \rightarrow C(X)$ for the homomorphism given by the inductive limit system. Without loss of generality, we may assume that $\mathcal{G} \subset \imath_{m}\left(C\left(Y_{m}\right)\right)$ for some $m \geq 1$. Let $\mathcal{G}^{\prime} \subset C\left(Y_{m}\right)$ be a finite subset such that $\imath_{m}\left(\mathcal{G}^{\prime}\right)=\mathcal{G}$. We may further assume that $\mathcal{P} \subset\left[\imath_{m}\right]\left(\underline{K}\left(C\left(Y_{m}\right)\right)\right)$ and $\mathcal{P}^{\prime} \subset \underline{K}\left(C\left(Y_{m}\right)\right)$ is a finite subset such that $\left[\imath_{m}\right]\left(\mathcal{P}^{\prime}\right)=\mathcal{P}$. As defined, we also assume that $\left(\delta, \mathcal{G}^{\prime}\right)$ is a $K K$-triple for $C\left(Y_{m}\right)$.

Let $\imath_{m}\left(C\left(Y_{m}\right)\right) \cong C(Y)$, where $Y$ is a compact subset of $Y_{m}$. Note that $\imath_{m}$ induces an embedding $\imath: C(Y) \rightarrow C(X)$. Denote by $s: X \rightarrow Y$ the surjective continuous map given by $\imath$, i.e, $\imath(f)(y)=f(s(y))$ for all $f \in C(Y)$.

Suppose that $Y_{m}$ is a finite disjoint union of connected finite $\mathrm{CW}$ complexes $Z_{1}, Z_{2}, \ldots, Z_{l}$. One can choose $\xi_{i} \in Z_{i}$ such that $\xi_{i} \in Y, i=1,2, \ldots, l$. There are $s_{1}, s_{2}, \ldots, s_{k} \in \cup_{i=1}^{l} K_{0}\left(C\left(Z_{i} \backslash\left\{\xi_{i}\right\}\right)\right)$ such that $\left[\imath_{m}\right]\left(s_{i}\right)=g_{i}, i=1,2, \ldots, k$. Write $K_{0}\left(C\left(Z_{i} \backslash\left\{\xi_{i}\right\}\right)\right)=\mathbb{Z}^{k(i)} \oplus G_{i}$, where $G_{i}$ is the torsion subgroup. Since $K_{0}(\mathbb{C})=\mathbb{Z}$ and $K_{1}(\mathbb{C})=\{0\}$, any homomorphism from $K_{i}\left(C\left(Y_{m}\right)\right)$ into $K_{i}(\mathbb{C})$ vanishes on $\operatorname{Tor}\left(K_{i}\left(C\left(Y_{m}\right)\right)\right), i=0,1$. To simplify the notation, without loss of generality, we may assume that $s_{1}, s_{2}, \ldots, s_{k}$ are the standard generators for $\oplus_{i=1}^{l} \mathbb{Z}^{(k(i)}$. We may assume that $\mathcal{G}_{i}^{\prime} \subset C\left(Z_{i}\right)$ is a finite subset such that $\oplus_{i=1}^{l} \mathcal{G}_{i}^{\prime}=\mathcal{P}^{\prime}$ and $\mathcal{P}_{i}^{\prime} \subset \underline{K}\left(C\left(Y_{m}\right)\right)$ is a finite subset such that $\oplus_{i=1}^{l} \mathcal{P}_{i}^{\prime}=\mathcal{P}^{\prime}$.

Applying 10.2 of [21] to each component $Z_{i}$, we obtain an integer $N_{i}\left(\delta, \mathcal{G}_{i}^{\prime}, \mathcal{P}_{i}^{\prime}\right)$ given by 10.2 of [21]. Let $N(\delta, \mathcal{G}, \mathcal{P})=\sum_{i=1}^{l} N_{i}\left(\delta, \mathcal{G}_{i}^{\prime}, \mathcal{P}_{i}^{\prime}\right)$.

Now let $L: C(X) \rightarrow B$ be a $\delta$ - $\mathcal{G}$-multiplicative contractive completely positive linear map. Put $L^{\prime}=L \circ \imath_{m}: C\left(Y_{m}\right) \rightarrow M_{n}$. Let $\kappa \in \operatorname{Hom}_{\Lambda}\left(\underline{K}\left(C\left(Y_{m}\right)\right), \underline{K}(B)\right)$ be given by $L^{\prime}$. Let $\kappa_{1} \in$ $K K(B, \mathbb{C})$ be given by a point-evaluation, if $B=M_{n}\left(C([0,1])\right.$, or $\kappa_{1}$ is given by the identity, if $B=M_{n}$. In either cases, one may view $\kappa_{1}$ is an identity on $\underline{K}(B)=\underline{K}(\mathbb{C})$. Put

$$
K=\max \left\{\left|\kappa\left(s_{j}\right)\right|: j=1,2, \ldots, k\right\} .
$$

It follows from 10.2 of [21] that there exists an integer $N(K) \geq 1$ and unital $\delta$ - $\mathcal{G}^{\prime}$-multiplicative contractive completely positive linear map $L_{0}^{\prime}: C\left(Y_{m}\right) \rightarrow M_{K(n)}$ such that

$$
\begin{aligned}
\frac{N(K)}{\max \{K, 1\}} & \leq N(\delta, \mathcal{G}, \mathcal{P}) \text { and } \\
{\left.\left[L_{0}^{\prime}\right]\right|_{\underline{K}\left(C_{0}\left(Z_{i} \backslash\left\{\xi_{i}\right\}\right)\right)} } & =-\left.\left(\kappa_{1} \times \kappa\right)\right|_{\underline{K}\left(C_{0}\left(Z_{i} \backslash\left\{\xi_{i}\right\}\right)\right)}, i=1,2, \ldots, l .
\end{aligned}
$$

If $N \geq N(K) / n$, by adding some point-evaluation, if necessary, we may assume that $L_{0}^{\prime}$ maps $C\left(Y_{m}\right)$ into a $C^{*}$-subalgebra $D \cong M_{n N}$ and $D$ is a $C^{*}$-subalgebra of $M_{N}(B)$ with $1_{D}=1_{M_{N}(B)}$. Then, viewing $L_{0}^{\prime}$ maps $C\left(Y_{m}\right)$ into $M_{N}(B)$,

$$
\kappa+\left.\left[L_{0}^{\prime}\right]\right|_{\underline{K}\left(C_{0}\left(Z_{i} \backslash\left\{\xi_{i}\right\}\right)\right)}=0 .
$$


There is a point-evaluation $h_{0}: C\left(Y_{m}\right) \rightarrow M_{n+N}(B)$ at $\left\{\xi_{1}, \xi_{2}, \ldots, \xi_{l}\right\}$ such that

$$
\left[L^{\prime} \oplus L_{0}^{\prime}\right]=\left[h_{0}\right] .
$$

We may write

$$
h_{0}(f)=\sum_{i=1}^{l} f\left(\xi_{i}\right) p_{i} \text { for all } f \in C\left(Y_{m}\right),
$$

where $p_{1}, p_{2}, \ldots, p_{l}$ are mutually orthogonal projections in $M_{N+1}(B)$. There is a unital contractive completely positive linear map $L_{0}: C(X) \rightarrow M_{N}(B)$ such that

$$
\left.L_{0} \circ \imath_{m}\right|_{C\left(Y_{m}\right)}=L_{0}^{\prime} .
$$

Note that $L_{0}$ is $\delta$-G-multiplicative. Define $H: C(X) \rightarrow M_{N+1}(B)$ by

$$
H(f)=\sum_{i=1}^{l} f\left(s\left(\xi_{i}\right)\right) p_{i} \text { for all } f \in C(X) .
$$

Then

$$
\left.\left[L \oplus L_{0}\right]\right|_{\mathcal{P}}=\left.[H]\right|_{\mathcal{P}}
$$

Lemma 4.8. Let $X$ be a compact metric space, let $\epsilon>0$, let $\mathcal{F} \subset C(X)$ be a finite subset. There exists a finite subset $\left\{x_{1}, x_{2}, \ldots, x_{m}\right\} \subset X(m \geq 1)$ satisfying the following: for any unital homomorphism $h_{0}: C(X) \rightarrow C\left([0,1], M_{n}\right)$ with finite dimensional range,

$$
\left\|\left(h_{0} \oplus h_{1}\right)(f)-\sum_{i=1}^{m} f\left(x_{i}\right) p_{i}\right\|<\epsilon \text { for all } f \in \mathcal{F},
$$

where $h_{1}: C(X) \rightarrow C\left([0,1], M_{(m-1) n}\right)$ is a unital homomorphism with finite dimensional range and $\left\{p_{1}, p_{2}, \ldots, p_{m}\right\}$ is a set of mutually orthogonal rank $n$ projections.

Proof. Let $\eta>0$ such that

$$
\left|f(x)-f\left(x^{\prime}\right)\right|<\epsilon / 4 \text { for all } f \in \mathcal{F} \text {, }
$$

provided that $\operatorname{dist}\left(x, x^{\prime}\right)<\eta$. Let $\left\{x_{1}, x_{2}, \ldots, x_{m}\right\}$ be an $\eta$-dense subset of $X$. Suppose that $h_{0}: C(X) \rightarrow C\left([0,1], M_{n}\right)$ is a unital homomorphism with finite dimensional range. Then there are $y_{1}, y_{2}, \ldots, y_{n} \in X$ and mutually orthogonal rank one projections $e_{1}, e_{2}, \ldots, e_{n}$ such that

$$
h_{0}(f)=\sum_{i=1}^{n} f\left(y_{i}\right) e_{i} \text { for all } f \in C(X) .
$$

Divide $\left\{y_{1}, y_{2}, \ldots, y_{n}\right\}$ into $N$ disjoint subsets $Y_{1}, Y_{2}, \ldots, Y_{N}$ with $1 \leq N \leq m$ such that

$$
\operatorname{dist}\left(y_{i}, x_{j}\right)<\eta,
$$

if $y_{i} \in Y_{j}$. Let $E_{j}=\sum_{y_{i} \in Y_{j}} e_{i}$ and denote by $R_{j}$ the rank of $E_{j}, j=1,2, \ldots, N$. Choose mutually orthogonal projections $q_{1}, q_{2}, \ldots, q_{m} \in C\left([0,1], M_{(m-1) n}\right)$ such that rank of $q_{j}=n-R_{j}, j=$ $1,2, \ldots, N$ and rank $q_{j}=n$ if $N<j \leq n$. Note $\sum_{j=1}^{m} q_{j}=1_{M_{(m-1) n}}$. Define $h_{1}: C(X) \rightarrow$ $C\left([0,1], M_{(m-1) n}\right)$ by

$$
h_{1}(f)=\sum_{j=1}^{n} f\left(x_{j}\right) q_{j} \text { for all } f \in C(X) .
$$


Let $p_{j}=E_{j}+q_{j}$ if $1 \leq j \leq N$ and $p_{j}=q_{j}$ if $N<j \leq m$. Note that $p_{j}$ has rank $n$ for $j=1,2, \ldots, m$. One then checks that

$$
\left\|\left(h_{0} \oplus h_{1}\right)(f)-\sum_{k=1}^{m} f\left(x_{k}\right) p_{k}\right\|<\epsilon \text { for all } f \in \mathcal{F} .
$$

Lemma 4.9. Let $X$ be a compact metric space, let $\mathcal{P} \subset \underline{K}(C(X))$ be a finite subset and let $G$ be the subgroup generated by $\mathcal{P}$. Suppose $\Delta:(0,1) \rightarrow(0,1)$ is a nondecreasing function, $\eta>0$ and $\lambda_{1}, \lambda_{2}>0$ are given. Suppose that $g_{1}, g_{2}, \ldots, g_{k}$ are generators of $G \cap \operatorname{ker} \rho_{C(X)}$.

Suppose that $L, \Lambda: C(X) \rightarrow A$ (for some unital separable simple $C^{*}$-algebra with tracial rank at most one) are two $\delta$-G-multiplicative contractive completely positive linear maps for which $[L]\left(g_{i}\right)$ and $[\Lambda]\left(g_{i}\right)$ are well defined $(i=1,2, \ldots, k)$, where $\delta$ is a positive number and $\mathcal{G}$ is a finite subset of $C(X)$,

$$
\left|\tau\left([L]\left(g_{i}\right)\right)\right|<\sigma \text { and }\left|\tau\left([\Lambda]\left(g_{i}\right)\right)\right|<\sigma \text { for all } \tau \in T(A), \quad i=1,2, \ldots, k,
$$

for some $1>\sigma>0$, and

$$
\mu_{\tau \circ L}\left(O_{r}\right) \geq \Delta(r), \quad \mu_{\tau \circ \Lambda}\left(O_{r}\right) \geq \Delta(r)
$$

for all $\tau \in T(A)$ and for all $r \geq \eta$.

Then, for any $\epsilon>0$ and any finite subset $\mathcal{F}$, any mutually orthogonal projections $e_{1}, e_{2}, \ldots, e_{N}$, any finite subset $\mathcal{H} \subset A$ and $R_{0}>1$, there exists a projection $p \in A$ and a unital $C^{*}$-subalgebra $B=\oplus_{j=1}^{m} C\left(X_{j}, M_{r(j)}\right)$, where $X_{j}=[0,1]$, or $X_{j}$ is a single point, with $1_{B}=p$, mutually orthogonal projections $e_{1}^{\prime}, e_{2}^{\prime}, \ldots, e_{N}^{\prime} \in B$ and a unital $(\delta+\epsilon)-\mathcal{G}$-multiplicative contractive completely positive linear maps $\psi_{1}, \psi_{2}: C(X) \rightarrow B$ such that

$$
\begin{aligned}
& \left\|L(f)-\left[(1-p) L(f)(1-p)+\psi_{1}(f)\right]\right\|<\epsilon, \\
& \left\|\Lambda(f)-\left[(1-p) \Lambda(f)(1-p)+\psi_{2}(f)\right]\right\|<\epsilon \text { for all } f \in \mathcal{F}, \\
& \tau(1-p)<\eta, \quad \tau\left(e_{i}^{\prime}\right) \geq \min \left\{\left(1-\lambda_{1}\right) \tau\left(e_{i}\right): \tau \in T(A)\right\} \text { for all } \tau \in T(A) \\
& r(j) \geq R_{0}, \quad j=1,2, \ldots, k, \\
& \left\|p e_{i} p-e_{i}^{\prime}\right\|<\epsilon, t_{j, x}\left(e_{i}^{\prime}\right) \geq \min \left\{\left(1-\lambda_{1}\right) \tau\left(e_{i}\right): \tau \in T(A)\right\} \\
& \left|t_{j, x}\left(\left[\psi_{1}\right]\left(g_{i}\right)\right)\right|<\left(1+\lambda_{1}\right) \sigma,\left|t_{j, x}\left(\left[\psi_{2}\right]\left(g_{i}\right)\right)\right|<\left(1+\lambda_{1}\right) \sigma \\
& \quad j=1,2, \ldots, k \text { and } x \in X_{j} \\
& \mu_{t_{j, x} \circ \psi_{1}}\left(O_{r}\right) \geq\left(1-\lambda_{1}\right) \Delta\left(r / 2\left(1+\lambda_{2}\right)\right), \quad \mu_{t_{j, x} \circ \psi_{2}}\left(O_{r}\right) \geq\left(1-\lambda_{1}\right) \Delta\left(r / 2\left(1+\lambda_{2}\right)\right)
\end{aligned}
$$

for all $r \geq 2\left(1+\lambda_{2}\right) \eta$ (We use $t_{j, x}$ for $\tau_{j, x} \otimes \operatorname{Tr}_{R}$ on $B \otimes M_{R}$, where $t_{j, x}(f)=t \circ f(x)$ for all $f \in C\left(X_{j}, M_{r(j)}\right)$, for all $x \in X_{j}$ and $t$ is the normalized trace on $M_{r(j)}$ and $\operatorname{Tr}_{R}$ is the standard trace on $M_{R}$.)

Moreover, for any $\epsilon_{0}>0$, one may assume that

$$
\|p a-a p\|<\epsilon_{0} \text { and pap } \epsilon_{\epsilon_{0}} B \text { for all } a \in \mathcal{H} .
$$

If furthermore, $\left.[L]\right|_{\mathcal{P}}=\left.[\Lambda]\right|_{\mathcal{P}}$ (in $K K(C(X), A)$, then, by taking smaller $\delta$ and larger $\mathcal{G}$, depending only on $\mathcal{P}$, one may further require that

$$
\left.\left[\psi_{1}\right]\right|_{\mathcal{P}}=\left.\left[\psi_{2}\right]\right|_{\mathcal{P}} \text { in } K K(C(X), B) .
$$


Proof. The proof is a modification of that of Lemma 9.7 of [21]. We repeat many arguments here. Let $p_{j}, q_{j} \in M_{R}(C(X))$ such that

$$
\left[p_{j}\right]-\left[q_{j}\right]=g_{j}, \quad j=1,2, \ldots, k,
$$

for some integer $R \geq 1$. There exists of a sequence of projections $p_{n} \in A$ such that

$$
\lim _{n \rightarrow \infty}\left\|c p_{n}-p_{n} c\right\|=0 \text { for all } c \in A
$$

and there exists a sequence of $C^{*}$-subalgebras $B_{n}=\oplus_{j=1}^{m(n)} C\left(X_{j, n}, M_{r(j, n)}\right)$ (where $X_{j, n}=[0,1]$ or $X$ is a single point) with $1_{B_{n}}=p_{n}$ such that

$$
\begin{aligned}
& \lim _{n \rightarrow \infty} \operatorname{dist}\left(p_{n} c p_{n}, B_{n}\right)=0 \text { and } \\
& \lim _{n \rightarrow \infty} \sup _{\tau \in T(A)}\left\{\tau\left(1-p_{n}\right)\right\}=0 .
\end{aligned}
$$

Moreover, by 3.3 of [15], we may also assume that $r(j, n) \geq R_{0}$ for all $j$. For sufficiently large $n$, there exists a contractive completely positive linear map $L_{n}^{\prime}: p_{n} A p_{n} \rightarrow B_{n}$ such that

$$
\lim _{n \rightarrow \infty}\left\|L_{n}^{\prime}(a)-p_{n} a p_{n}\right\|=0 \text { for all } a \in A
$$

(see 2.3 .9 of [11]). There are (see 2.55 and 2.5.6 of [11]) mutually orthogonal projections $e_{i, n} \in B_{n}$ such that

$$
\lim _{n \rightarrow \infty}\left\|p_{n} e_{i} p_{n}-e_{i, n}^{\prime}\right\|=0, \quad i=1,2, \ldots, N
$$

We have

$$
\begin{aligned}
& \lim _{n \rightarrow \infty}\left\|L(f)-\left[\left(1-p_{n}\right) L(f)\left(1-p_{n}\right)+L_{n}^{\prime} \circ L(f)\right]\right\|=0 \text { and } \\
& \lim _{n \rightarrow \infty}\left\|\Lambda(f)-\left[\left(1-p_{n}\right) \Lambda(f)\left(1-p_{n}\right)+L_{n}^{\prime} \circ \Lambda(f)\right]\right\|=0 \text { for all } f \in C(X) .
\end{aligned}
$$

Define $L_{n, R}^{\prime}: M_{R}(A) \rightarrow M_{R}(A)$ by $L_{n}^{\prime} \otimes \mathrm{id}_{M_{R}}$ and $L_{R}: M_{R}(C(X)) \rightarrow M_{R}(A)$ by $L_{R}=L \otimes \mathrm{id}_{M_{R}}$. Suppose that there exists a subsequence $\left\{n_{k}\right\},\left\{j_{k}\right\}$ and $\left\{x_{k}\right\} \in[0,1]$ such that

$$
\left|t_{j_{k}, x_{k}}\left(L_{n_{k}, R}^{\prime} \circ L_{R}\left(p_{i}-q_{i}\right)\right)\right| \geq\left(1+\lambda_{1}\right) \sigma
$$

for all $k$. Define a state $T_{k}: A \rightarrow \mathbb{C}$ by $T_{k}(a)=t_{j_{k}, x_{k}}(a), k=1,2, \ldots$ Let $T$ be a limit point. Note $T_{k}\left(1_{A}\right)=1$. Therefore $T$ is a state on $A$. Then, by (e 4.160),

$$
\left|T\left([L]\left(g_{i}\right)\right)\right| \geq\left(1+\lambda_{1}\right) \sigma .
$$

However, it is easy to check that $T$ is a tracial state. This contradicts with (e 4.144). Put $\psi_{1}=L_{n}^{\prime} \circ L$ and $\psi_{2}=L_{n}^{\prime} \circ \Lambda$ for some large $n$. Then we have shown (for the choice of large $n$ ) that (e4.147), (e4.148) and (e4.153) hold.

A similar argument shows that, for some sufficiently large $n$,

$$
t_{j, x}\left(e_{i, n}^{\prime}\right) \geq \min \left\{\left(1-\lambda_{1}\right) \tau\left(e_{i}\right): \tau \in T(A)\right\},
$$

$i=1,2, \ldots, N$, for all $x \in X_{j}$ and $j=1,2, \ldots, m(n)$.

Moreover, a similar argument shows that, for any finitely many $f_{1}, f_{2}, \ldots, f_{N} \in C(X)$ such that $0<f_{i} \leq 1, i=1,2, \ldots, N$, we may assume (by choosing large $n$ ) that

$$
\begin{array}{r}
t_{j, x} \circ \psi_{1}\left(f_{k}\right) \geq\left(1-\lambda_{1} / 2\right) \min \left\{\tau\left(L\left(f_{k}\right)\right): \tau \in T(A)\right\} \text { and } \\
t_{j, x} \circ \psi_{2}\left(f_{k}\right) \geq\left(1-\lambda_{1} / 2\right) \min \left\{\tau\left(\Lambda\left(f_{k}\right)\right): \tau \in T(A)\right\}
\end{array}
$$


for all $x \in X_{j}, j=1,2, \ldots, m$. By choosing sufficiently many (but finitely many) $f_{j}{ }^{\prime} s$, using the argument in the proof of 3.2 , we may assume that

$$
\mu_{t_{j, x} \circ \psi_{i}}\left(O_{r}\right) \geq\left(1-\lambda_{1}\right) \Delta\left(\eta / 2\left(1+\lambda_{2}\right)\right)
$$

for all $r \geq 2\left(1+\lambda_{2}\right) \eta$ and for all $x \in X, j=1,2, \ldots, m$ and $i=1,2$.

So the first part of the lemma follows by choosing $B$ to be $B_{n}, p$ to be $p_{n}$ and $\psi_{1}$ to be $L_{n}^{\prime} \circ L$ and $\psi_{2}$ to be $L_{n}^{\prime} \circ \Lambda$ for some sufficiently large $n$. Note, by (e 4.155) and (e 4.156), for any $\epsilon_{0}>0$ and any finite subset $\mathcal{H}$, we can assume that

$$
\|p a-a p\|<\epsilon_{0} \text { and } p a p \epsilon_{\epsilon_{0}} B
$$

for all $a \in \mathcal{H}$.

To see the last part of the lemma holds, taking a commutative $C^{*}$-algebra $C$ and considering the maps $L \otimes \operatorname{id}_{M_{l}(C)}$ and $\Lambda \otimes \operatorname{id}_{M_{l}(C)}$ from $C(X) \otimes M_{l}(C)$ into $A \otimes M_{l}(C)$. There is $K_{0} \geq 1$ such that, if $x \in \operatorname{Tor}\left(K_{i}(C(X))\right) \cap G$, then $K_{0} x=0$. Let $C_{1}, C_{2}, \ldots, C_{K_{0}}$ ! be unital commutative $C^{*}$-algebra such that $K_{0}\left(C_{j}\right)=\mathbb{Z} \oplus \mathbb{Z} / j \mathbb{Z}$ and $K_{1}\left(C_{j}\right)=\{0\}, j=1,2, \ldots, K_{0}$ !. Let $l \geq 1$ be an integer such that a set of generators of $K_{0}\left(C(X) \otimes C_{j}\right) \cap G$ and $K_{1}\left(C(X) \otimes C_{j}\right) \cap G$ can be represented by projections and unitaries in $M_{l}\left(C(X) \otimes C_{j}\right)=C(X) \otimes C_{j} \otimes M_{l}, j=1,2, \ldots, K_{0}$ !

Choose $0<\epsilon_{1}<\epsilon$ and a finite subset $\mathcal{F}_{1} \supset \mathcal{F}$ (which depends on $K_{0}$ and $l$ above). Then one applies the first part of the lemma for this $\epsilon_{1}$ and $\mathcal{F}_{1}$. For a finite subset of projections $E_{1}, E_{2}, \ldots, E_{K} \in C(X) \otimes C_{j} \otimes M_{l}$, if in addition that $\left.[L]\right|_{\mathcal{P}}=\left.[\Lambda]\right|_{\mathcal{P}}$ (with sufficiently small $\delta$ and sufficiently large $\mathcal{G}$ ), there are partial isometries $W_{1}, W_{2}, \ldots, W_{K} \in A \otimes C_{j} \otimes M_{l+R}$ for some integer $R \geq 0$ such that

$$
W_{i} W_{i}^{*}=E_{i}^{\prime} \oplus \operatorname{id}_{M_{R}\left(C_{j}\right)} \text { and } W_{i}^{*} W_{i}=E_{i}^{\prime \prime} \oplus \operatorname{id}_{M_{R}\left(C_{j}\right)},
$$

where $E_{i}^{\prime}$ and $E_{i}^{\prime \prime}$ are two projections such that

$$
\left\|E_{i}^{\prime}-L \otimes \operatorname{id}_{M_{l}\left(C_{j}\right)}\left(E_{i}\right)\right\|<1 / 16 \text { and }\left\|E_{i}^{\prime \prime}-\Lambda \otimes \operatorname{id}_{M_{l}\left(C_{j}\right)}\left(E_{i}\right)\right\|<1 / 16,
$$

$i=1,2, \ldots, K$.

Fix $\epsilon_{0}>0$. One then chooses a large $\mathcal{H}$ so that

$$
\|p a-a p\|<\epsilon_{0} \text { and } p a p \epsilon_{\epsilon_{0}} B
$$

imply that

$$
\begin{aligned}
& \left\|\left(p \otimes \operatorname{id}_{M_{l+R}\left(C_{j}\right)}\right) W_{i}-W_{i}\left(p \otimes \operatorname{id}_{M_{R}\left(C_{j}\right)}\right)\right\|<\epsilon_{1}, \\
& \left\|\left(p \otimes \operatorname{id}_{M_{l+R}\left(C_{j}\right)}\right)\left(E_{i}^{\prime} \otimes \operatorname{id}_{M_{R}\left(C_{j}\right)}\right)-\left(E_{i}^{\prime} \otimes \operatorname{id}_{M_{R}\left(C_{j}\right)}\right)\left(p \otimes \operatorname{id}_{M_{l+R}\left(C_{j}\right)}\right)\right\|<\epsilon_{1} \text { and } \\
& \left\|\left(p \otimes \operatorname{id}_{M_{l+R}\left(C_{j}\right)}\right)\left(E_{i}^{\prime \prime} \otimes \operatorname{id}_{M_{R}\left(C_{j}\right)}\right)-\left(E_{i}^{\prime \prime} \otimes \operatorname{id}_{M_{R}\left(C_{j}\right)}\right)\left(p \otimes \operatorname{id}_{M_{l+R}\left(C_{j}\right)}\right)\right\|<\epsilon_{1}, \\
& \left\|\left(p \otimes \operatorname{id}_{M_{l}\left(C_{j}\right)}\right) E_{i}^{\prime}-E_{i}^{\prime}\left(p \otimes \operatorname{id}_{M_{l}\left(C_{j}\right)}\right)\right\|<\epsilon_{1} \text { and } \\
& \left\|\left(p \otimes \operatorname{id}_{M_{l}\left(C_{j}\right)}\right) E_{i}^{\prime \prime}-E_{i}^{\prime \prime}\left(p \otimes \operatorname{id}_{M_{l}\left(C_{j}\right)}\right)\right\|<\epsilon_{1}
\end{aligned}
$$

as well as

$$
\begin{aligned}
& \left(p \otimes \operatorname{id}_{M_{l}\left(C_{j}\right)}\right) E_{i}^{\prime}\left(p \otimes \operatorname{id}_{M_{l}\left(C_{j}\right)}\right) \epsilon_{\epsilon_{1}} B \otimes M_{l}\left(C_{j}\right), \\
& \left(p \otimes \operatorname{id}_{M_{l}\left(C_{j}\right)}\right) E_{i}^{\prime \prime}\left(p \otimes \operatorname{id}_{M_{l}\left(C_{j}\right)}\right) \epsilon_{\epsilon_{1}} B \otimes M_{l}\left(C_{j}\right), \\
& \left(p \otimes \operatorname{id}_{M_{l+R}\left(C_{j}\right)}\right) W_{i}\left(p \otimes \operatorname{id}_{M_{l+R}\left(C_{i}\right)}\right)\left(p \otimes \operatorname{id}_{M_{l+R}\left(C_{j}\right)}\right) \epsilon_{\epsilon_{1}} B \otimes M_{l+R}\left(C_{j}\right), \\
& \left(p \otimes \operatorname{id}_{M_{l+R}\left(C_{j}\right)}\right)\left(E_{i}^{\prime} \otimes \operatorname{id}_{M_{R}\left(C_{j}\right)}\right)\left(p \otimes \operatorname{id}_{M_{l+R}\left(C_{j}\right)}\right) \epsilon_{\epsilon_{1}} B \otimes M_{l+R}\left(C_{j}\right) \text { and } \\
& \left(p \otimes \operatorname{id}_{M_{l+R}\left(C_{j}\right)}\right)\left(E_{i}^{\prime \prime} \otimes \operatorname{id}_{M_{R}\left(C_{j}\right)}\right)\left(p \otimes \operatorname{id}_{M_{l+R}\left(C_{j}\right)}\right) \epsilon_{\epsilon_{1}} B \otimes M_{l+R}\left(C_{j}\right) .
\end{aligned}
$$


It follows that (with small $\epsilon_{1}$ ) there are projections $e_{i}^{\prime}, e_{i}^{\prime \prime} \in B \otimes M_{l}\left(C_{j}\right)$ such that

$$
\begin{gathered}
\left\|e_{i}^{\prime}-\left(p \otimes \operatorname{id}_{M_{l}\left(C_{j}\right)}\right) E_{i}^{\prime}\left(p \otimes \operatorname{id}_{M_{l}\left(C_{j}\right)}\right)\right\|<2 \epsilon_{1},\left\|e_{i}^{\prime \prime}-\left(p \otimes \operatorname{id}_{M_{l}\left(C_{j}\right)}\right) E_{i}^{\prime \prime}\left(p \otimes \operatorname{id}_{M_{l}\left(C_{j}\right)}\right)\right\|<2 \epsilon_{1} \text { and } \\
{\left[e_{i}\right]=\left[e_{i}^{\prime}\right] \text { in } K_{0}(B) .}
\end{gathered}
$$

Therefore, one has

$$
\left[\psi_{1}\right]\left(\left[E_{i}\right]\right)=\left[\psi_{2}\right]\left(\left[E_{i}\right]\right), \quad i=1,2, \ldots, K .
$$

From this, one concludes that one may require that

$$
\left.\left[\psi_{1} \otimes \operatorname{id}_{\left.C_{j}\right)}\right]\right|_{K_{0}\left(C(X) \otimes C_{j}\right) \cap G}=\left.\left[\psi_{2} \otimes \operatorname{id}_{C_{j}}\right]\right|_{K_{0}\left(C(X) \otimes C_{j}\right) \cap G},
$$

$j=1,2, \ldots, K_{0}$ !. A similar argument shows that one may also require that

$$
\left.\left[\psi_{1} \otimes \operatorname{id}_{C_{j}}\right]\right|_{K_{1}\left(C(X) \otimes C_{j}\right) \cap G}=\left.\left[\psi_{2} \otimes \operatorname{id}_{C_{j}}\right]\right|_{K_{1}\left(C(X) \otimes C_{j}\right) \cap G},
$$

$j=1,2, \ldots, K_{0}$ !. It follows that one may require that

$$
\left[\psi_{1}\right]=\left[\psi_{2}\right] \text { in } K K(C(X), B) .
$$

\section{The main results}

Lemma 5.1. Let $X$ be a compact metric space, let $\epsilon>0, \epsilon_{0}>0$, let $\left\{x_{1}, x_{2}, \ldots, x_{m}\right\} \subset X$, let $\mathcal{F} \subset C(X)$ be a finite subset and let $\Delta:(0,1) \rightarrow(0,1)$ be an increasing map with $\lim _{t \rightarrow 0} \Delta(t)=0$. Let $\mathcal{P} \subset \underline{K}(C(X))$ be a finite subset, $K \geq 1$ be an integer and let $\eta_{0}>0$. Then, there exists $\eta>0, \delta>0$, a finite subset $\mathcal{G} \subset C(X)$ satisfying the following: For any unital $\delta$-G-multiplicative contractive completely positive linear maps $L, \Lambda: C(X) \rightarrow$ A for some unital separable simple $C^{*}$-algebra $A$ with tracial rank at most one for which

$$
\left.[\Lambda]\right|_{\mathcal{P}}=\left.[L]\right|_{\mathcal{P}} \text { and } \mu_{\tau \circ L}\left(O_{r}\right), \mu_{\tau \circ L}\left(O_{r}\right) \geq \Delta(r)
$$

for all open balls $O_{r}$ with radius $1>r \geq \eta$, and, for any $\epsilon_{00}>0$ and any finite subset $\mathcal{H} \subset A$, there exist mutually orthogonal projections $P_{1}, P_{2}, P_{3}, p_{1}, p_{2}, \ldots, p_{m} \in A$ with $P_{1} \oplus P_{2} \oplus P_{3} \oplus \sum_{i=1}^{m} p_{i}=1_{A}$,

$$
\begin{array}{r}
\tau\left(P_{3}\right)>1-\epsilon_{0} \text { for all } \tau \in T(A) \text { and } \\
K\left[P_{1} \oplus P_{2}\right] \leq\left[p_{i}\right], \quad i=1,2, \ldots, m,
\end{array}
$$

and there exists a unital $\epsilon-\mathcal{F}$-multiplicative contractive completely positive linear map $\psi: C(X) \rightarrow$ $P_{2} \mathrm{BP}_{2}$ whose range contained in a finite dimensional $C^{*}$-subalgebra, unital $\epsilon-\mathcal{F}$-multiplicative contractive completely positive linear maps $H_{1}, H_{2}: C(X) \rightarrow P_{3} B P_{3} \subset P_{3} A P_{3}$, where $P_{2}, P_{3}$, $p_{1}, p_{2}, \ldots, p_{m} \in B, B=\oplus_{j=1}^{N} B_{j}$, and $B_{j}=C\left(X_{j}, M_{r(j)}\right)\left(X_{j}=[0,1]\right.$, or $X_{j}$ is a point) with

$$
\left.\left[H_{1}\right]\right|_{\mathcal{P}}=\left.\left[H_{2}\right]\right|_{\mathcal{P}}=\left.\left[h_{0}\right]\right|_{\mathcal{P}}
$$

for some unital homomorphism $h_{0}: C(X) \rightarrow C$, where $C=P_{3} B P_{3}, C=\oplus_{j=1}^{N} C_{j}$ and $C_{j}=$ $C\left(X_{j}, M_{r^{\prime}(j)}\right)$, and a unitary $W \in A$ such that

$$
\begin{gathered}
\| L(f)-\left[\left(P_{1} L(f) P_{1} \oplus H_{1}(f) \oplus \psi(f) \oplus \sum_{i=1}^{m} f\left(x_{i}\right) p_{i}\right] \|<\epsilon\right. \\
\left\|\operatorname{Ad} W \circ \Lambda(f)-\left[P_{1}(\operatorname{Ad} W \circ \Lambda)(f) P_{1} \oplus H_{2}(f) \oplus \psi(f) \oplus \sum_{i=1}^{m} f\left(x_{i}\right) p_{i}\right]\right\|<\epsilon
\end{gathered}
$$

for all $f \in \mathcal{F}$,

$$
\mu_{t_{j, x} \circ H_{i}}\left(O_{r}\right) \geq \Delta(r / 3) / 2 \text { and } t\left(P_{2}+\sum_{i=1}^{m} p_{i}\right)<\epsilon_{0}
$$


for all $r \geq \eta_{0}, x \in X_{j}$, where $t_{j, x}$ is the composition of the point-evaluation at $x$ and the normalized trace on $M_{r^{\prime}(j)}, j=1,2, \ldots, k$, and for all $t \in T(B)$, and

$$
\left\|P_{1} a-a P_{1}\right\|<\epsilon_{00}, \quad\left(1-P_{1}\right) a\left(1-P_{1}\right) \epsilon_{\epsilon_{00}} B \text { for all } a \in \mathcal{H} \cup L(\mathcal{F}) \cup \Lambda(\mathcal{F}),
$$

where $1_{B}=1-P_{1}$, Moreover,

$$
\left.\left[P_{1} L P_{1}\right]\right|_{\mathcal{P}}=\left.\left[P_{1}(\operatorname{Ad} W \circ \Lambda) P_{1}\right]\right|_{\mathcal{P}}
$$

Proof. Let $\epsilon, \epsilon_{0},\left\{x_{1}, x_{2}, \ldots, x_{m}\right\} \subset X$, a finite subset $\mathcal{F} \subset X$, a finite subset $\mathcal{P} \subset \underline{K}(C(X)), \Delta$, $K \geq 1$ and $\eta_{0}>0$ be as described. We may assume that $(\epsilon, \mathcal{F}, \mathcal{P})$ is a $K L$-triple for $C(X)$ and $0<\epsilon_{0}, \epsilon<1 / 16$.

Let $\delta_{1}>0$ (in place of $\delta$ ), $\mathcal{G}_{1} \subset C(X)$ be a finite subset (in place of $\mathcal{G}$ ), $\mathcal{P}_{1} \subset \underline{K}(C(X)$ ) (in place of $\mathcal{P}$ ) be a finite subset and $K_{1}$ be an integer (in place of $L$ ) for $\min \left\{\epsilon / 16, \epsilon_{0} / 16\right\}$ and $\mathcal{F}$ required by 4.3 .

We may also assume, without loss of generality, that $\mathcal{P} \subset \mathcal{P}_{1}$ and $\left(\delta_{1}, \mathcal{G}_{1}, \mathcal{P}_{1}\right)$ forms a $K L$ triple. We may further assume that, if $L^{\prime}, L^{\prime \prime}: C(X) \rightarrow C$ (for any unital $C^{*}$-algebra $C$ ) are $\epsilon-\mathcal{G}_{1}$-multiplicative contractive completely positive linear maps and

$$
\left\|L^{\prime}(f)-L^{\prime \prime}(f)\right\|<\epsilon \text { for all } f \in \mathcal{G}_{1}
$$

then

$$
\left.\left[L^{\prime}\right]\right|_{\mathcal{P}_{1}}=\left.\left[L^{\prime \prime}\right]\right|_{\mathcal{P}_{1}} .
$$

Let $G$ be the subgroup generated by $\mathcal{P}_{1}$ and let $s_{1}, s_{2}, \ldots, s_{k_{0}}$ be a set of generators of $G \cap$ $\operatorname{ker} \rho_{C(X)}$.

Let $\epsilon_{2}=\min \left\{\epsilon / 64, \epsilon_{0} / 64, \delta_{1} / 2, \delta^{\prime} / 2\right\}$ and $\mathcal{G}_{2}=\mathcal{F} \cup \mathcal{G}_{1} \cup \mathcal{G}^{\prime}$.

Let $N_{1}=N\left(\epsilon_{2}, \mathcal{G}_{2}, \mathcal{P}_{1}\right)$ be as in 4.7 where $\delta$ is replaced by $\epsilon_{2}, \mathcal{G}$ is replace by $\mathcal{G}_{2}$ and $\mathcal{P}$ is replaced by $\mathcal{P}_{1}$.

Let $N_{2}$ (in place $m$ ) and $\left\{y_{1}, y_{2}, \ldots, y_{N_{2}}\right\}$ (in place of $\left\{x_{1}, x_{2}, \ldots, x_{m}\right\}$ ) be as in 4.8 for $\epsilon_{2}$ (in place $\epsilon$ ) and $\mathcal{G}_{2}$ (in place of $\mathcal{F}$ ). One may assume that $N_{2}>m$ and $y_{j}=x_{j}, j=1,2, \ldots, m$.

Choose $\eta^{\prime}>0$ satisfying the following:

$$
\left|f(x)-f\left(x^{\prime}\right)\right|<\epsilon_{2} / 16
$$

for all $f \in \mathcal{G}_{2}$, if $\operatorname{dist}\left(x, x^{\prime}\right)<2 \eta^{\prime}$. Moreover, we may assume that

$$
O_{4 \eta^{\prime}}\left(y_{j}\right) \cap O_{4 \eta^{\prime}}\left(y_{i}\right)=\emptyset \text { if } i \neq j .
$$

Choose $\eta^{\prime \prime}>0$ such that $\eta^{\prime \prime}<\eta_{0} / 4$ and

$$
\Delta\left(\eta^{\prime \prime}\right)<\frac{\Delta\left(\eta_{0} / 4\right)}{256 N_{2}}
$$

Choose

$$
\eta=\min \left\{\eta_{1} / 4, \eta_{2} / 4, \eta^{\prime} / 4, \eta^{\prime \prime} / 4\right\} .
$$

Let $\delta_{2}>0$ (in place of $\delta$ ) and let $\mathcal{G}_{3} \subset C(X)$ be a finite subset required by Lemma 9.6 of 21] for $\epsilon_{2} / 2$ (in place of $\epsilon$ ), $\mathcal{G}_{2}$ (in place of $\mathcal{F}$ ), $\eta$ and $1 / 256$ (in place of $r$ ).

Choose $\bar{K}$ to be an integer which is greater than the integer $K$ given by this lemma. We may assume that $K>4$. Choose $d>0$ such that

$$
16 d \bar{K} N_{1} N_{2}^{2}\left(K_{1}+1\right)<\epsilon_{0} \Delta(\eta) / 2^{11} .
$$


It follows from 4.6 that there are $\delta_{3}>0$ and a finite subset $\mathcal{G}_{4} \subset C(X)$ such that, for any unital $\delta_{3}-\mathcal{G}_{4}$-multiplicative contractive completely positive linear map $\Psi: C(X) \rightarrow C$ (for any unital $C^{*}$-algebra $C$ with $T(C) \neq \emptyset$ ),

$$
\left|\tau\left([\Psi]\left(s_{i}\right)\right)\right|<d / 8 \text { for all } \tau \in T(C) .
$$

Let $\delta=\min \left\{\epsilon_{2} / 4, \delta_{2} / 4, \delta_{3} / 4, \delta_{4}\right\}$ and $\mathcal{G}=\cup_{i=1}^{5} \mathcal{G}_{i}$.

Now suppose that $L, \Lambda: C(X) \rightarrow A$, where $A$ is a unital simple $C^{*}$-algebra with tracial rank at most one, satisfy the assumptions of the theorem for the above $\delta, \mathcal{G}, \eta$ and $\Delta$.

By Theorem 9.6 of [21] and by the choice of $\eta$, there exists projections $Q_{1}, Q_{2} \in A$ and two sets of mutually orthogonal projections $\left\{E_{1}, E_{2}, \ldots, E_{N_{2}}\right\}$ in $\left(1-Q_{1}\right) A\left(1-Q_{1}\right)$ and $\left\{E_{1}^{\prime}, E_{2}^{\prime}, \ldots, E_{N_{2}}^{\prime}\right\}$ in $\left(1-Q_{2}\right) A\left(1-Q_{2}\right)$ such that $\sum_{i=1}^{N_{2}} E_{i}=1-Q_{1}, \sum_{i=1}^{N_{2}} E_{i}^{\prime}=\left(1-Q_{2}\right)$,

$$
\begin{array}{r}
\left.\| L(g)-\left[Q_{1} L(g) Q_{1} \oplus \sum_{i=1}^{N_{2}} g\left(y_{i}\right) E_{i}\right)\right] \|<\epsilon_{2} / 2, \\
\left\|\Lambda(g)-\left[Q_{2} \Lambda(g) Q_{2} \oplus \sum_{i=1}^{N_{2}} g\left(y_{i}\right) E_{i}^{\prime}\right]\right\|<\epsilon_{2} / 2
\end{array}
$$

for all $g \in \mathcal{G}_{2}$,

$$
\begin{aligned}
& \Delta(\eta)>\tau\left(E_{i}\right) \geq\left(1-1 / 2^{12}\right) \Delta(\eta) \text { and } \\
& \Delta(\eta)>\tau\left(E_{i}^{\prime}\right) \geq\left(1-1 / 2^{12}\right) \Delta(\eta), \quad i=1,2, \ldots, N_{2},
\end{aligned}
$$

for all $\tau \in T(A)$. Since $A$ has tracial rank at most one (see Lemma 9.9 of [21]), there is, for each $i$, a projection $q_{i} \leq E_{i}$ such that $\left[q_{i}\right] \leq\left[E_{i}^{\prime}\right]$ and

$$
\tau\left(q_{i}\right) \geq\left(1-1 / 2^{11}\right) \Delta(\eta) \text { for all } \tau \in T(A) .
$$

Let $q_{i}^{\prime} \leq E_{i}^{\prime}$ such that $\left[q_{i}\right]=\left[q_{i}^{\prime}\right], i=1,2, \ldots, N_{2}$. Let $Q_{0}=1-\sum_{i=1}^{N_{2}} q_{i}$ and $Q_{0}^{\prime}=1-\sum_{i=1}^{N_{2}} q_{i}^{\prime}$. Then, we have that

$$
\begin{gathered}
\left\|L(g)-\left[Q_{1} L(g) Q_{1} \oplus \sum_{i=1}^{N_{2}} g\left(y_{i}\right)\left(E_{i}-q_{i}\right)+\sum_{i=1}^{N_{2}} g\left(y_{i}\right) q_{i}\right]\right\|<\epsilon_{2} / 2 \\
\| \Lambda(g)-\left[Q_{2} \Lambda(g) Q_{2} \oplus \sum_{i=1}^{N_{2}} g\left(y_{i}\right)\left(E_{i}^{\prime}-q_{i}^{\prime}\right)+\sum_{i=1}^{N_{2}} g\left(y_{i}\right) q_{i}^{\prime} \|<\epsilon_{2} / 2\right.
\end{gathered}
$$

for all $g \in \mathcal{G}_{2}$. Since $\left[q_{i}\right]=\left[q_{i}^{\prime}\right], i=1,2, \ldots, N_{2}$, there is a unitary $W_{1} \in A$ such that

$$
W_{1}^{*} q_{i}^{\prime} W_{1}=q_{i}, \quad i=1,2, \ldots, N_{2} \text { and } W_{1}^{*} Q_{0}^{\prime} W_{1}=Q_{0} .
$$

Define $L_{1}: C(X) \rightarrow Q_{0} A Q_{0}$ by $L_{1}(f)=Q_{1} L(f) Q_{1} \oplus \sum_{i=1}^{N_{2}} f\left(y_{i}\right)\left(E_{i}-q_{i}\right)$ for all $f \in C(X)$. Define $\Lambda_{1}: C(X) \rightarrow Q_{0} A Q_{0}$ by $\Lambda_{1}(f)=W_{1}^{*}\left(Q_{2} \Lambda(f) Q_{1} \oplus \sum_{i=1}^{N_{2}} f\left(y_{i}\right)\left(E_{i}^{\prime}-q_{i}^{\prime}\right)\right) W_{1}$ for all $f \in C(X)$. Then $L_{1}$ and $\Lambda_{1}$ are $\epsilon_{2}-\mathcal{G}_{4}$-multiplicative and

$$
\begin{gathered}
\left\|L(g)-\left[L_{1}(g)+\sum_{i=1}^{N_{2}} g\left(y_{i}\right) q_{i}\right]\right\|<\epsilon_{2} / 2 \text { and } \\
\left\|\operatorname{Ad} W_{1} \circ \Lambda(g)-\left[\Lambda_{1}(g)+\sum_{i=1}^{N_{2}} g\left(y_{i}\right) q_{i}\right]\right\|<\epsilon_{2} / 2
\end{gathered}
$$


for all $g \in \mathcal{G}_{2}$. Note that

$$
\left[L_{1}\right]\left(s_{i}\right)=[L]\left(s_{i}\right) \text { and }\left[\Lambda_{1}\right]\left(s_{i}\right)=[\Lambda]\left(s_{i}\right), \quad i=1,2, \ldots, k_{0} .
$$

We compute that

$$
\mu_{\tau \circ Q_{1} L Q_{1}}\left(O_{r}\right) \geq \Delta(r)-N_{2} \Delta(\eta) \geq 255 \Delta(r) / 256
$$

for all $\tau \in T(A)$ and $r \geq \eta_{0} / 4$. It follows that

$$
\mu_{\tau \circ L_{1}}\left(O_{r}\right) \geq 255 \Delta(r) / 256
$$

for all $\tau \in T(A)$ and $r \geq \eta_{0} / 4$. Similarly,

$$
\mu_{\tau \circ \Lambda_{1}}\left(O_{r}\right) \geq 255 \Delta(r) / 256
$$

for all $\tau \in T(A)$ and $r \geq \eta_{0} / 4$.

Let $\theta<\frac{\epsilon_{0} \Delta(\eta / 2)}{8 N_{1} N_{2} K}$. Let $\epsilon_{00}>0$ and let $\mathcal{H} \subset A$ be a finite subset. Define

$$
\mathcal{H}_{1}=\mathcal{H} \cup L(\mathcal{G}) \cup L_{1}(\mathcal{G}) \cup \Lambda_{1}(\mathcal{G}) \cup \operatorname{Ad} W_{1} \circ \Lambda(\mathcal{G}) \cup\left\{P_{1}, q_{1}, q_{2}, \ldots, q_{N_{2}}\right\} .
$$

Let $0<\delta_{0}<\min \left\{\epsilon_{2} / 2, \delta / 4, \epsilon_{00}\right\}$ and put

$$
\begin{array}{r}
L^{\prime}(f)=L_{1}(f) \oplus \sum_{i=1}^{N_{2}} f\left(y_{i}\right) q_{i} \text { and } \\
\Lambda^{\prime}(f)=\Lambda_{1}(f) \oplus \sum_{i=1}^{N_{2}} f\left(y_{i}\right) q_{i}
\end{array}
$$

for all $f \in C(X)$. Since $A$ has tracial rank at most one, by 4.9, there exists a projection $Q_{3} \in A$ and $B=\oplus_{j=1}^{N} B_{j}$ with $1_{B}=Q_{3}$, where $B_{j}=M_{r(j)}\left(C\left(X_{j}\right)\right)$ and $X_{j}=[0,1]$, or $X_{j}$ is a point, such that

$$
\begin{array}{r}
\left\|Q_{3} a-a Q_{3}\right\|<\delta_{0}, \quad Q_{3} a Q_{3} \in_{\delta_{0}} B \text { for all } a \in \mathcal{H}_{1}, \\
\left\|L^{\prime}(f)-\left[\left(1-Q_{3}\right) L^{\prime}(f)\left(1-Q_{3}\right) \oplus L_{3}(f)\right]\right\|<\epsilon_{2} / 2 N_{2}, \\
\left\|\Lambda^{\prime}(f)-\left[\left(1-Q_{3}\right) \Lambda(f)\left(1-Q_{3}\right) \oplus \Lambda_{3}(f)\right]\right\|<\epsilon_{2} / 2 N_{2} \text { for all } f \in \mathcal{G}, \\
\tau\left(1-Q_{3}\right)<\theta \text { for all } \tau \in T(A), \\
\left|T_{j, x}\left(\left[L_{3}\right]\left(s_{i}\right)\right)\right|<(1+1 / 128)(d / 8), \quad \mid T_{j, x}\left(\left[\Lambda_{3}\right]\left(s_{i}\right) \mid<(1+1 / 128)(d / 8)\right. \\
\mu_{T_{j, x} \circ L_{3}}\left(O_{r}\right) \geq 3 \Delta(r / 3) / 4, \quad \mu_{T_{j, x} \circ \Lambda_{3}}\left(O_{r}\right) \geq 3 \Delta(r / 3) / 4
\end{array}
$$

for all $r \geq \eta_{0}$, and for all $j$ and $x \in X_{j}$, where $T_{j, x}$ is the normalized trace of $M_{r(j)}$ at $x \in X_{j}$, and

$$
r(j)>\frac{2^{15} \bar{K} N_{1} N_{2}^{2}\left(K_{1}+1\right)}{\epsilon_{0} \Delta(\eta / 2)}, j=1,2, \ldots, N
$$

Moreover,

$$
\left.\left[L_{3}\right]\right|_{\mathcal{P}_{1}}=\left.\left[\Lambda_{3}\right]\right|_{\mathcal{P}_{1}} \text { in } K L(C(X), B) .
$$

Therefore

$$
\left\|Q_{3} L^{\prime}(f) Q_{3}-L_{3}(f)\right\|<\epsilon_{2} / 2 N_{2} \text { and }\left\|Q_{3} \Lambda^{\prime}(f) Q_{3}-\Lambda_{3}(f)\right\|<\epsilon_{2} / 2 N_{2}
$$


for all $f \in \mathcal{G}$. By 4.9, we further obtain mutually orthogonal projections $e_{1}, e_{2}, \ldots, e_{N_{2}} \in B$ such that

$$
\begin{aligned}
& \left\|L_{3}(f)-\left[E L_{3}(f) E \oplus \sum_{i=1}^{N_{2}} f\left(y_{i}\right) e_{i}\right]\right\|<\epsilon_{2} / 2 \\
& \left\|\Lambda_{3}(f)-\left[E \Lambda_{3}(f) E \oplus \sum_{i=1}^{N_{2}} f\left(y_{i}\right) e_{i}\right]\right\|<\epsilon_{2} / 2
\end{aligned}
$$

for all $f \in \mathcal{G}$, where $E=1_{B}-\sum_{i=1}^{N_{2}} e_{i}$. Moreover we require that

$$
\Delta(\eta) / 2>T_{j, x}\left(e_{i}\right) \geq(1-1 / 250) \Delta(\eta), \Delta(\eta) / 2>\tau\left(e_{i}\right) \geq(1-1 / 250) \Delta(\eta)
$$

for all $x \in X_{j}$, where $T_{j, x}$ is the normalized trace evaluated at $x$, and for all $\tau \in T(A), j=$ $1,2, \ldots, N_{2}$.

Define $L_{4}=E L_{3} E$ and $\Lambda_{4}=E \Lambda_{3} E$. We compute, by (e 5.203) and (e 5.209)

$$
\begin{aligned}
& \mu_{T_{j, x} \circ L_{4}}\left(O_{r}\right) \geq 3 \Delta(r / 3) / 4-N_{2} \Delta(\eta) \geq \Delta(r / 3) / 2 \text { and } \\
& \mu_{T_{j, x} \circ \Lambda_{4}}\left(O_{r}\right) \geq \Delta(r / 3) / 2 .
\end{aligned}
$$

for all $r \geq \eta_{0}$ and for all $x \in X_{j}$ and $j=1,2, \ldots, N$.

We compute that

$$
\left[L_{4}\right]\left(s_{j}\right)=\left[\Lambda_{4}\right]\left(s_{j}\right)=[L]\left(s_{j}\right)=[\Lambda]\left(s_{j}\right), j=1,2, \ldots, k_{0} .
$$

Put $C_{j}^{\prime}=E\left(M_{r(j)}\left(C\left(X_{j}\right)\right) E=M_{r^{\prime \prime}(j)}\left(C\left(X_{j}\right)\right)\right.$, where $r^{\prime \prime}(j) \leq r(j)$, put $L_{4, j}=\pi_{j} \circ L_{4}$ and $\Lambda_{4, j}=\pi_{j} \circ \Lambda_{4}$, where $\pi_{j}: E B E \rightarrow E B_{j} E$ is the projection, $j=1,2, \ldots, N$.

Let

$$
d_{j}=\max \left\{\left|T_{j, x}\left(\left[L_{4}\right]\left(s_{k}\right)\right)\right|: k=1,2, \ldots, k_{0}\right\},
$$

$j=1,2, \ldots, N$. Note that $d_{j} \leq d / 4, j=1,2, \ldots, N$.

It follows from 4.7 that there exist unital $\epsilon_{2}-\mathcal{G}_{2}$-multiplicative contractive completely positive linear maps $L_{0, j}, \bar{L}_{0, j}: C(X) \rightarrow M_{J_{j}}\left(C\left(X_{j}\right)\right)$ whose ranges are contained in finite dimensional $C^{*}$-subalgebras, where

$$
J_{j}=d_{j} N_{1} r(j) \leq d N_{1} r(j)
$$

such that

$$
\left.\left[L_{4, j} \oplus L_{0, j}\right]\right|_{\mathcal{P}_{1}}=\left.\left[H_{0, j}\right]\right|_{\mathcal{P}_{1}} \text { and }\left.\left[L_{0, j} \oplus \bar{L}_{0, j}\right]\right|_{\mathcal{P}_{1}}=\left.\left[h_{0, j}\right]\right|_{\mathcal{P}_{1}}
$$

for some unital homomorphisms $H_{0, j}: C(X) \rightarrow M_{r^{\prime \prime}(j)+J_{j}}\left(C\left(X_{j}\right)\right)$ and $h_{0, j}: C(X) \rightarrow M_{2 J_{j}}\left(C\left(X_{j}\right)\right)$ with finite dimensional range.

By applying 4.3, we obtain a unital homomorphism $h_{1, j}: C(X) \rightarrow M_{2 J_{j} K_{1}}\left(C\left(X_{j}\right)\right)$ with finite dimensional range and a unital homomorphism $H_{1, j}: C(X) \rightarrow M_{2 J_{j}\left(K_{1}+1\right)}\left(C\left(X_{j}\right)\right)$ with finite dimensional range such that

$$
\left\|\left(L_{0, j} \oplus \bar{L}_{0, j} \oplus h_{1, j}\right)(f)-H_{1, j}(f)\right\|<\min \left\{\epsilon / 16, \epsilon_{0} / 16\right\} \text { for all } g \in \mathcal{F} .
$$

It follows from 4.8 that there are mutually orthogonal rank $2 J_{j}\left(K_{1}+1\right)$ projections $q_{i, j}^{\prime} \in$ $M_{N_{2} 2 J_{1}\left(K_{1}+1\right)}\left(C\left(X_{j}\right)\right)$ and a unital homomorphism $h_{2, j}: C(X) \rightarrow M_{\left(N_{2}-1\right)\left(2 J_{1}\left(K_{1}+1\right)\right.}\left(C_{j}\right)$ with finite dimensional range such that

$$
\left\|H_{1, j}(f) \oplus h_{2, j}(f)-\sum_{i=1}^{N_{2}} f\left(y_{i}\right) q_{i, j}^{\prime}\right\|<\epsilon_{2} \text { for all } f \in \mathcal{F},
$$


$j=1,2, \ldots, N$.

There is, for each $i$ and $j$, by (e 5.209) and (e 5.184), a projection $p_{i, j}^{\prime} \leq e_{i}$ such that

$$
\epsilon_{0} / 128 N_{2} \geq T_{j, x}\left(p_{i, j}^{\prime}\right) \geq \epsilon_{0} / 256 N_{2} \geq 16 d \bar{K} N_{1} N_{2}\left(K_{1}+1\right)
$$

for $x \in X_{j}, j=1,2, \ldots, N$ and $i=1,2, \ldots, N_{2}$.

Put $L_{0}=\sum_{j=1}^{N} L_{0, j}, \bar{L}_{0}=\sum_{j=1}^{N} \bar{L}_{0, j}, h_{i}=\sum_{j=1}^{N} h_{i, j}, i=1,2$. There is a projection $q_{i, j} \leq p_{i, j}^{\prime}$ in $B_{j}$ such that $\left[q_{i, j}\right]=\left[q_{i, j}^{\prime}\right]$ in $K_{0}\left(B_{j}\right), j=1,2, \ldots, N$. Put $p_{i}^{\prime \prime}=\sum_{j=1}^{N} q_{i, j}, i=1,2, \ldots N_{2}$. Then

$$
T_{j, x}\left(p_{i}^{\prime \prime}\right)<2 J_{j}\left(K_{1}+1\right) / r(j) \text { for all } x \in X_{j} .
$$

Thus we obtain a unitary $W_{0} \in B$ such that

$$
\left\|\operatorname{Ad} W_{0} \circ\left(L_{0} \oplus \bar{L}_{0} \oplus h_{1} \oplus h_{2}\right)(f)-\sum_{i=1}^{N_{2}} f\left(y_{i}\right) p_{i}^{\prime \prime}\right\|<\epsilon_{2}+\epsilon / 16
$$

for all $f \in \mathcal{F}$.

Now define

$$
H_{1}(f)=L_{4}(f) \oplus \operatorname{Ad} W_{0} \circ L_{0} \oplus h_{1}(f) \oplus h_{2}(f) \oplus \sum_{k=1}^{m} f\left(y_{k}\right)\left(e_{i}-p_{i}^{\prime}\right) \oplus \sum_{j=m+1}^{N_{2}} f\left(y_{j}\right)\left(e_{i}-p_{i}^{\prime \prime}\right)
$$

for all $f \in C(X), \psi=\operatorname{Ad} W_{0} \circ \bar{L}_{0}$. Let $P_{1}=\left(1-Q_{3}\right), P_{2}=W_{0}^{*} \bar{L}_{0}\left(1_{C(X)}\right) W_{0}, P_{3}=H_{1}\left(1_{C(X)}\right)$. $p_{j}=p_{j}^{\prime}-p_{j}^{\prime \prime}, j=1,2, \ldots, N_{2}$. Then we estimate that, by (e 5.207) and (e 5.219),

$$
\begin{aligned}
& \left\|L_{3}(f)-\left(H_{1} \oplus \psi(f) \oplus \sum_{j=1}^{m} f\left(x_{j}\right) p_{j}\right)\right\| \\
& \quad<\left\|L_{3}(f)-\left(L_{4}(f) \oplus \sum_{i=1}^{N_{2}} f\left(y_{j}\right) e_{j}\right)\right\|+ \\
& \left.\| L_{4}(f) \oplus \sum_{j=1}^{N_{2}} f\left(y_{j}\right) e_{j}-L_{4}(f) \oplus \operatorname{Ad} W_{0}\left(L_{0} \oplus \bar{L}_{0} \oplus h_{1} \oplus h_{2}\right)(f) \oplus \sum_{j=1}^{m} f\left(y_{j}\right) p_{j}\right) \| \\
& \quad<\epsilon_{2} / 2+\epsilon_{2}+\epsilon / 16=3 \epsilon_{2} / 2+\epsilon / 16
\end{aligned}
$$

for all $f \in \mathcal{F}$. It follows from (e 5.191), (e 5.196), (e 5.199) and (e 5.223) that

$$
\begin{aligned}
& \| L(f)-\left[P_{1} L(f) P_{1} \oplus \psi(f) \oplus \sum_{j=1}^{m} f\left(x_{j}\right) p_{j} \oplus H_{1}(f) \|\right. \\
< & \left\|L(f)-L^{\prime}(f)\right\|+\left\|L^{\prime}(f)-\left(1-Q_{3}\right) L^{\prime}(f)\left(1-Q_{3}\right) \oplus L_{3}(f)\right\| \\
& +\left\|\left(1-Q_{3}\right) L^{\prime}(f)\left(1-Q_{3}\right) \oplus L_{3}(f)-P_{1} L(f) P_{1} \oplus L_{3}(f)\right\| \\
& +\left\|P_{1} L(f) P_{1} \oplus L_{3}(f)-\left[P_{1} L(f) P_{1} \oplus H_{1}(f) \oplus \psi(f) \oplus \sum_{j=1}^{m} f\left(x_{j}\right) p_{j}\right]\right\| \\
< & \epsilon_{2} / 2+\epsilon_{2} / 2+3 \epsilon_{2} / 2+\epsilon / 16<\epsilon
\end{aligned}
$$

for all $f \in \mathcal{F}$. Define

$$
H_{2}(f)=\Lambda_{4} \oplus \operatorname{Ad} W_{0} \circ L_{0} \oplus h_{1}(f) \oplus h_{2}(f) \oplus \sum_{k=1}^{N_{2}} f\left(y_{k}\right)\left(e_{i}-p_{i}^{\prime}\right) \oplus \sum_{j=m+1}^{N_{2}} f\left(y_{j}\right)\left(e_{j}-p_{j}^{\prime \prime}\right) .
$$


Similarly, we also have

$$
\left\|\operatorname{Ad} W_{1} \circ \Lambda(f)-\left[P_{1}\left(\operatorname{Ad} W_{1} \circ \Lambda(f)\right) P_{1} \oplus \psi(f) \oplus \sum_{j=1}^{m} f\left(x_{j}\right) p_{j} \oplus H_{2}(f)\right]\right\|<\epsilon
$$

for all $f \in \mathcal{F}$.

Note also that, (by (e5.184) and (e5.218)

$$
\begin{aligned}
\frac{\epsilon_{0} \Delta(\eta)}{128 N_{2}} \geq T_{j, x}\left(p_{i}\right) & =T_{j, x}\left(p_{j}^{\prime}-p_{j}^{\prime \prime}\right) \geq \frac{\epsilon_{0} \Delta(\eta)}{256 N_{2}}-2 J_{j}\left(K_{1}+1\right) / r(j) \\
& \geq 14 \bar{K} d N_{1} N_{2}\left(K_{1}+1\right)
\end{aligned}
$$

for all $\tau \in T(A)$ and $i=1,2, \ldots, N_{2}$. Therefore, by (e 5.201),

$$
\tau\left(p_{i}\right) \geq(1-\theta) 14 d \bar{K} N_{1} N_{2}\left(K_{1}+1\right) \text { for all } \tau \in T(A) .
$$

Note that, by (e5.213),

$$
T_{j, x}\left(P_{2}\right) \leq d_{j} N_{1} \text { and } \tau\left(P_{1}\right)=\tau\left(1-Q_{3}\right)<\theta
$$

for all $x \in X_{j}, j=1,2, \ldots, N$ and for all $\tau \in T(A)$. It follows that

$$
\tau\left(p_{i}\right)>K \tau\left(P_{2}\right)+K \tau\left(P_{1}\right) \text { for all } \tau \in T(A), \quad i=1,2, \ldots, m .
$$

This gives (e5.177). To obtain (e 5.176), we note, by (e5.217) and (e5.184) that

$$
\begin{aligned}
\tau\left(P_{1}\right)+\tau\left(P_{2}\right)+\sum_{i=1}^{m} \tau\left(p_{i}\right) & <\theta+d N_{1}+\frac{\epsilon_{0}}{128 N_{2}} m \\
& <\frac{\epsilon_{0} \Delta(\eta / 2)}{4 N_{1} N_{2} \tilde{K}}+\frac{\epsilon_{0} \Delta(\eta / 2)}{256 \tilde{K}}+\frac{\epsilon_{0}}{128}<\epsilon_{0} / 2
\end{aligned}
$$

for all $\tau \in T(A)$. We also have that

$$
T_{j, x}\left(P_{2}\right)+\sum_{i=1}^{m} T_{j, x}\left(p_{i}\right)<\epsilon_{0} / 2 \text { for all } x \in X_{j}
$$

Thus

$$
t\left(P_{2}+\sum_{i=1}^{m} p_{i}\right)<\epsilon_{0} / 2
$$

for all $t \in T(B)$. This implies (e 5.176$)$. We write $C=P_{3} B P_{3}=\oplus_{j=1}^{N} C_{j}$, where $C_{j}=$ $M_{r^{\prime}(j)}\left(C\left(X_{j}\right)\right), j=1,2, \ldots, N$. Finally, from (e 5.210) and (e 5.211),

$$
\mu_{t_{j, x} \circ H_{i}}\left(O_{r}\right) \geq \Delta(r / 3) / 2
$$

for all $r \geq \eta_{0}$ and $x \in X_{j}$, where $t_{j, x}$ is the normalized trace of $M_{r^{\prime}(j)}$ evaluated at $x \in X_{j}$, $j=1,2, \ldots, N$ and $i=1,2$. The lemma follows.

Lemma 5.2. Let $C$ be a separable unital $C^{*}$-algebra with $T(C) \neq \emptyset$, let $\mathcal{U} \subset U_{c}\left(K_{1}(C)\right)$ be a finite subset, $\mathcal{F} \subset C$ be a finite subset and let $\lambda>0$. There exists $\delta>0$ and a finite subset 
$\mathcal{G} \subset C$ satisfying the following: Suppose that $L_{1}, L_{2}: C \rightarrow A$ (for some unital $C^{*}$-algebra $A$ ) are two $\delta$-G-multiplicative contractive completely positive linear maps such that

$$
\operatorname{dist}\left(\overline{\left\langle L_{1}(u)\right\rangle}, \overline{\left\langle L_{2}(u)\right\rangle}\right) \leq \Gamma
$$

for all $u \in \mathcal{U}$ and for some $\Gamma>0$. There exists a finite subset $\mathcal{H} \subset A$ and $\sigma>0$ such that, if $p \in A$ is a projection such that

$$
\|p a-a p\|<\sigma, p a p \in_{\sigma} B \text { for all } a \in \mathcal{H},
$$

where $1_{B}=p$ and $B \subset p A p$ is a unital $C^{*}$-subalgebra, and $\Lambda_{1}, \Lambda_{2}: C \rightarrow B$ are two $2 \delta$ - $\mathcal{G}$ multiplicative contractive completely positive linear maps such that

$$
\left\|p L_{i}(g) p-\Lambda_{i}(g)\right\|<\sigma \text { for all } g \in \mathcal{G},
$$

then

$$
\operatorname{dist}\left(\overline{\left\langle\Lambda_{1}(u)\right\rangle}, \overline{\left\langle\Lambda_{2}(u)\right\rangle}\right) \leq \Gamma+\lambda(\text { in } B)
$$

for all $u \in \mathcal{U}$.

Moreover,

$$
\left|\tau \circ \Lambda_{1}(f)-\tau \circ \Lambda_{2}(f)\right| \leq \lambda+\max \left\{\left|t \circ L_{1}(f)-\tau \circ L_{2}(f)\right|: f \in \mathcal{F}, t \in T(A)\right\}
$$

for all $f \in \mathcal{F}$ and $\tau \in T(B)$.

Proof. Let $\mathcal{U}$ and $\mathcal{F}$ be fixed. Then there is an integer $k \geq 1$ such that every element in $\mathcal{U}$ is represented by a unitary in $M_{k}(C)$. To simplify notation, replacing $A$ by $M_{k}(A)$, replacing $B$ by $M_{k}(B)$, and later replacing $L_{i}$ by $L_{i} \otimes \mathrm{id}_{M_{k}}, i=1,2$, without loss of generality, we may assume that $\mathcal{U}$ is actually in $U(A)$. We choose $\delta$ and $\mathcal{G}$ such that for any $2 \delta-\mathcal{G}$-multiplicative contractive completely positive linear maps $L$ from $C,\langle L(u)\rangle$ is well defined for all $u \in \mathcal{U}$.

Now let $L_{1}$ and $L_{2}$ be as described (for the above choice of $\delta$ and $\mathcal{G}$ ). Suppose that $\mathcal{U}=$ $\left\{u_{1}, u_{2}, \ldots, u_{m}\right\}$. Then there are $w_{1}, w_{2}, \ldots, w_{m} \in C U(A)$ such that

$$
\left\|\left\langle L_{1}\left(u_{i}\right)\right\rangle\left\langle L_{2}\left(u_{i}^{*}\right)\right\rangle-w_{i}\right\| \leq \Gamma+\lambda / 2
$$

It is clear that, if $\mathcal{H}$ is sufficiently large (containing at least $L_{1}(u)$ and $L_{2}(u)$ for all $u \in \mathcal{U}$ and many other elements in $U(A))$ and $\sigma$ is sufficiently small, $\left\langle p L_{i} p\left(u_{j}\right)\right\rangle$ are well defined and

$$
\left\|\left\langle p L_{i} p\left(u_{j}\right)\right\rangle-\left\langle\Lambda_{i}\left(u_{j}\right)\right\rangle\right\|<\lambda / 16
$$

$(j=1,2, \ldots, m$ and $i=1,2)$ and there are unitaries $v_{1}, v_{2}, \ldots, v_{m} \in C U(B)$ such that

$$
\left\|p w_{i} p-v_{i}\right\|<\lambda / 16, \quad i=1,2, \ldots, m .
$$

It follows that

$$
\operatorname{dist}\left(\overline{\left\langle\Lambda_{1}(u)\right\rangle}, \overline{\left\langle\Lambda_{2}(u)\right\rangle}\right) \leq \Gamma+\lambda
$$

for all $u \in \mathcal{U}$.

Similarly, for each $f \in \mathcal{F}$, there are $x_{1}(f), x_{2}(f), \ldots, x_{f(m)}(f) \in A$ such that

$$
\begin{gathered}
\left\|L_{1}(f)-\sum_{i=1}^{f(m)} x_{i}(f)^{*} x_{i}(f)\right\|<\lambda / 8 \text { and } \\
\left\|L_{2}(f)-\sum_{i=1}^{f(m)} x_{i}(f) x_{i}(f)^{*}\right\|<M+\lambda / 8
\end{gathered}
$$


where $M=\max \left\{\left|\tau \circ L_{1}(f)-\tau \circ L_{2}(f)\right|: f \in \mathcal{F}, \tau \in T(A)\right\}$ (see [1]). We compute that, with sufficiently large $\mathcal{H}$ and small $\sigma$, there are $y_{1}(f), y_{2}(f), \ldots, y_{f(m)}(f) \in B$ such that

$$
\begin{aligned}
& \left\|\Lambda_{1}(f)-\sum_{i=1}^{f(m)} y_{i}(f)^{*} y_{i}(f)\right\|<\lambda / 4 \text { and } \\
& \left\|\Lambda_{1}(f)-\sum_{i=1}^{f(m)} y_{i}(f)^{*} y_{i}(f)\right\|<M+\lambda / 4
\end{aligned}
$$

for all $f \in \mathcal{F}$. This implies that

$$
\left|\tau \circ \Lambda_{1}(f)-\tau \circ \Lambda_{2}(f)\right|<M+\lambda
$$

for all $f \in \mathcal{F}$ and for all $\tau \in T(B)$.

Theorem 5.3. Let $X$ be a compact metric space and let $\Delta:(0,1) \rightarrow(0,1)$ be a non-decreasing function with $\lim _{t \rightarrow 0} \Delta(t)=0$. Let $\epsilon>0$ and $\mathcal{F} \subset C(X)$ be a finite subset. Then there exists $\eta>$ $0, \delta>0$, a finite subset $\mathcal{G} \subset C(X)$, a finite subset $\mathcal{H} \subset C(X)_{\text {s.a. }}$, a finite subset $\mathcal{P} \subset \underline{K}(C(X))$, a finite subset $\mathcal{U} \subset U_{c}\left(K_{1}(C(X))\right), \gamma_{1}>0$ and $\gamma_{2}>0$ satisfying the following: Suppose that $L_{1}, L_{2}: C(X) \rightarrow A$ are two unital $\delta$-G-multiplicative contractive completely positive linear maps for some unital simple $C^{*}$-algebra $A$ of tracial rank at most one such that

$$
\begin{aligned}
& {\left.\left[L_{1}\right]\right|_{\mathcal{P}}=\left.\left[L_{2}\right]\right|_{\mathcal{P}}} \\
& \left|\tau \circ L_{1}(h)-\tau \circ L_{2}(h)\right|<\gamma_{1} \text { for all } h \in \mathcal{H} \text {, } \\
& \operatorname{dist}\left(\left\langle\overline{L_{1}(u)}\right\rangle,\left\langle\overline{L_{2}(u)}\right\rangle\right)<\gamma_{2} \text { for all } u \in \mathcal{U} \\
& \mu_{\tau \circ L_{i}}\left(O_{r}\right)>\Delta(r)
\end{aligned}
$$

for all $\tau \in T(A)$ and for all $r \geq \eta$. Then there exists a unitary $W \in A$ such that

$$
\left\|\operatorname{Ad} W \circ L_{1}(f)-L_{2}(f)\right\|<\epsilon \text { for all } f \in \mathcal{F} \text {. }
$$

Proof. Fix $\epsilon>0$ and a finite subset $\mathcal{F} \subset C(X)$. Let $\eta_{1}>0$ be as in 3.6 for $\epsilon / 2$ (in place of $\epsilon$ ) and $\mathcal{F}$. Let $\sigma_{1}=\Delta\left(\eta_{1} / 3\right) / 3$. Let $\eta_{2}>0$ be as in 3.6 for $\epsilon / 2$ (in place of $\epsilon$ ), $\mathcal{F}, \eta_{1}$ and $\sigma_{1}$. Let $\sigma_{2}=\Delta\left(\eta_{2} / 3\right) / 3$. Let $\eta_{3}>0$ be as in 3.6 for $\epsilon / 2$ (in place of $\epsilon$ ), $\mathcal{F}, \eta_{1}, \sigma_{1}, \eta_{2}$ and $\sigma_{2}$. Let $\sigma_{3}=\Delta\left(\eta_{3} / 3\right) / 3$. Let $\eta_{4}>0$ be as in 3.6 for $\epsilon / 2$ (in place of $\epsilon$ ), $\mathcal{F}, \eta_{1}, \sigma_{1}, \eta_{2}, \sigma_{2}, \eta_{3}$ and $\sigma_{3}$. Let $\sigma_{4}=\Delta\left(\eta_{4} / 3\right) / 3$.

Let $\gamma_{1}^{\prime}>0$ ( in place of $\gamma_{1}$ ), $\gamma_{2}^{\prime}>0$ (in place of $\gamma_{2}$ ), $\delta_{1}$ (in place of $\delta$ ), $\mathcal{G}_{1} \subset C(X)$ (in place of $\mathcal{G}$ ) be a finite subset, $\mathcal{P}_{1} \subset \underline{K}\left(C(X)\right.$ ) (in place of $\mathcal{P}$ ) be a finite subset, $\mathcal{H} \subset C(X)_{\text {s.a. }}$ be a finite subset and $\mathcal{U}_{1} \subset U_{c}\left(K_{1}(C(X))\right.$ ) (in place of $\mathcal{U}$ ) be a finite subset as required by 3.6 for $\epsilon / 2, \mathcal{F}, \eta_{i}$ and $\sigma_{i}(i=1,2,3,4)$. Let $N \geq 1$ be an integer such that every unitary in $\mathcal{U}_{1}$ is in $M_{N}(U(C(X)))$.

Let $\Delta_{1}=\Delta / 2$. Let $\delta_{2}>0$ (in place of $\delta$ ) and $\mathcal{G}_{2} \subset C(X)$ (in place of $\mathcal{G}$ ) be required by 3.4 for $\Delta_{1}$ (in place of $\Delta$ ), $\mathcal{U}_{1}$ (in place of $\mathcal{U}$ ), $\eta_{4} / 2$ (in place $\eta$ ), 15/16 (in place of $\lambda_{1}$ ) and 1/32 (in place of $\lambda_{2}$ ).

Let $\delta_{3}>0$ (in place of $\delta$ ), $\mathcal{G}_{3} \subset C(X)$ (in place of $\mathcal{G}$ ) be a finite subset, $\mathcal{P}_{2} \subset \underline{K}(C(X)$ ) (in place of $\mathcal{P}$ ) be a finite subset $\left\{x_{1}, x_{2}, \ldots, x_{m}\right\} \subset X, \mathcal{U}_{2} \subset U_{c}\left(K_{1}(C(X)\right.$ ) (in place of $\mathcal{U}$ ) and $K \geq 1$ (in place of $L$ ) be an integer required by 4.5 for $\epsilon / 2$ (in place of $\epsilon$ ), $\mathcal{F}$ and $\gamma_{2}^{\prime}$ (in place of $\lambda)$.

Let $\delta_{4}>0$ (in place of $\delta$ ), $\mathcal{G}_{4} \subset C(X)$ (in place of $\mathcal{G}$ ) be a finite subset required by Lemma 5.2 for $\gamma_{2}^{\prime} / 8$ (in place of $\lambda$ ), $\mathcal{U}_{1} \cup \mathcal{U}_{2}$ (in place of $\mathcal{U}$ ) and $\mathcal{H}$ (in place of $\mathcal{F}$ ). 
Let $\delta_{5}=\min \left\{\epsilon / 4, \delta_{i}: 1 \leq i \leq 4\right\} \mathcal{G}_{5}=\mathcal{F} \cup \cup_{i=1}^{4} \mathcal{G}_{i}, \mathcal{U}=\mathcal{U}_{1} \cup \mathcal{U}_{2}, \gamma_{1}=\gamma_{1}^{\prime} / 8$ and $\gamma_{2}=\gamma_{2}^{\prime} / 8$. Put $\epsilon_{0}=\min \left\{\gamma_{1}^{\prime} / 8 N, \gamma_{2}^{\prime} / 8 N\right\}$ and $\eta_{0}=\min \left\{\eta_{i} / 4: 1 \leq i \leq 4\right\}$.

Let $\eta>0, \delta_{6}>0$ (in place of $\delta$ ), $\mathcal{G}_{6} \subset C(X)$ (in place of $\mathcal{G}$ ) be a finite subset required by 5.1 for $\delta_{5}$ (in place of $\epsilon$ ), $\epsilon_{0},\left\{x_{1}, x_{2}, \ldots, x_{m}\right\}, \mathcal{G}_{5}$ (in place of $\mathcal{F}$ ), $\Delta, K$ and $\eta_{0}$.

Define $\delta=\min \left\{\delta_{6}, \delta_{5}\right\}, \mathcal{G}=\mathcal{G}_{6} \cup \mathcal{G}_{5}$ and $\mathcal{P}=\mathcal{P}_{1} \cup \mathcal{P}_{2}$.

Now suppose that $L_{1}, L_{2}: C(X) \rightarrow A$ are two unital $\delta$ - $\mathcal{G}$-multiplicative contractive completely positive linear maps, where $A$ is a unital simple $C^{*}$-algebra of tracial rank at most one, which satisfy the assumption for the above defined $\Delta, \eta, \mathcal{H}, \mathcal{U}, \gamma_{1}$ and $\gamma_{2}$.

Let $\mathcal{H}_{1} \subset A$ (in place of $\mathcal{H}$ ) be a finite subset and $\sigma>0$ for $L_{1}, L_{2}, \mathcal{U}, \lambda_{2}^{\prime} / 8$ (in place of $\Gamma$ ) and $\min \left\{\lambda_{1}^{\prime} / 8, \lambda_{2}^{\prime} / 8\right\}$ (in place of $\lambda$ ) (for $C=C(X)$ ) be required by 5.2. Let $\epsilon_{00}=\sigma$.

Let $\delta_{7}=\min \{\sigma / 2, \delta\}$.

By applying $\left[5.1\right.$, for $\mathcal{H}_{1}$ (in place of $\mathcal{H}$ ), there exist mutually orthogonal projections

$$
P_{1}, P_{2}, P_{3}, p_{1}, p_{2}, \ldots, p_{m} \in A
$$

with $P_{2}, P_{3}, p_{1}, p_{2}, \ldots, p_{m} \in B, P_{1}+P_{2}+P_{3}+\sum_{i=1}^{m} p_{i}=1_{A}$,

$$
\tau\left(P_{3}\right)>1-\epsilon_{0} \text { and } K\left(\left[P_{1}\right]+\left[P_{2}\right]\right) \leq\left[p_{i}\right], \quad i=1,2, \ldots, m,
$$

a unital $\delta_{5}-\mathcal{G}_{5}$-multiplicative contractive completely positive linear map $\psi: C(X) \rightarrow P_{2} B P_{2}$ whose range is contained in a finite dimensional $C^{*}$-subalgebra, unital $\delta_{5}-\mathcal{G}_{5}$-multiplicative contractive completely positive linear maps $H_{1}, H_{2}: C(X) \rightarrow P_{3} B P_{3} \subset P_{3} A P_{3}$, where $1_{B}=1-P_{1}$, $B=\oplus_{j=1}^{N} B_{j}, B_{j}=C\left(X_{j}, M_{r(j)}\right)\left(X_{j}=[0,1]\right.$, or $X_{j}$ is a point $)$ with

$$
\left.\left[H_{1}\right]\right|_{\mathcal{P}}=\left.\left[H_{2}\right]\right|_{\mathcal{P}}=\left.\left[h_{0}\right]\right|_{\mathcal{P}}
$$

for some unital homomorphism $h_{0}: C(X) \rightarrow B$ and a unitary $W_{0} \in A$ such that

$$
\begin{array}{r}
\left\|L_{1}(g)-\left[P_{1} L_{1}(g) P_{1} \oplus \psi(g) \oplus \sum_{i=1}^{m} g\left(x_{i}\right) p_{i} \oplus H_{1}(g)\right]\right\|<\delta_{7} \text { and } \\
\left\|\operatorname{Ad} W_{0} \circ L_{2}(g)-\left[P_{1}\left(\operatorname{Ad} W_{0} \circ L_{2}(g)\right) P_{1} \oplus \psi(g) \oplus \sum_{i=1}^{m} g\left(x_{i}\right) p_{i} \oplus H_{2}(g)\right]\right\|<\delta_{7}
\end{array}
$$

for all $g \in \mathcal{G}_{5}$,

$$
\mu_{t \circ H_{i}}\left(O_{r}\right) \geq \Delta(r / 3) / 2
$$

for all $r \geq \eta_{0}$ and for all $t \in T(C)$, where $C=P_{3} B P_{3}$,

$$
T\left(P_{2} \oplus \sum_{i=1}^{m} p_{i}\right)<\epsilon_{0}
$$

for all $T \in T(B)$, and,

$$
\begin{aligned}
& \left\|P_{1} a-a P_{1}\right\|<\epsilon_{00} \text { and } \\
& \left(1-P_{1}\right) a\left(1-P_{1}\right) \in_{\epsilon_{00}} B \text { for all } a \in \mathcal{H}_{1} \cup L_{1}\left(\mathcal{G}_{5} \cup L_{2}\left(\mathcal{G}_{5}\right) .\right.
\end{aligned}
$$

Moreover

$$
\left.\left[P_{1} L_{1} P_{1}\right]\right|_{\mathcal{P}}=\left.\left[P_{1} \operatorname{Ad} W_{0} \circ L_{2} P_{1}\right]\right|_{\mathcal{P}} .
$$


Put $\Psi_{1}=P_{1} L_{1} P_{1} \oplus \psi$ and $\Psi_{2}=P_{1}$ Ad $W_{0} \circ L_{2} P_{1} \oplus \psi$. By the choice of $\mathcal{H}_{1}$,

$$
\operatorname{dist}\left(\overline{\left\langle P_{1} L_{1} P_{1}(u)\right\rangle}, \overline{\left\langle P_{1} \operatorname{Ad} W_{0} \circ L_{2} P_{1}(u)\right\rangle}\right)<\gamma_{2}^{\prime} / 4+\gamma_{2}^{\prime} / 2=\lambda_{2}
$$

for all $u \in \mathcal{U}$. Let $D$ be a finite dimensional $C^{*}$-subalgebra of $P_{2} B P_{2}$ such that $\psi(C(X)) \subset D$. Then

$$
\langle\psi(u)\rangle \in C U\left(P_{2} B P_{2}\right) \text { for all } u \in \mathcal{U} \text {. }
$$

It follows that

$$
\operatorname{dist}\left(\overline{\left\langle\Psi_{1}(u)\right\rangle}, \overline{\left\langle\Psi_{2}(u)\right\rangle}\right)<\lambda_{2}
$$

for all $u \in \mathcal{U}$. By the choices of $K$ and $\left\{x_{1}, x_{2}, \ldots, x_{m}\right\}$, there exists a unitary

$$
W_{1} \in\left(P_{1}+P_{2}+\sum_{i=1}^{m} p_{i}\right) A\left(P_{1}+P_{2}+\sum_{i=1}^{m} p_{i}\right)
$$

such that

$$
\left\|W_{1}^{*}\left(\Psi_{2}(f) \oplus \sum_{i=1}^{m} f\left(x_{i}\right) p_{i}\right) W_{1}-\Psi_{1}(f) \oplus \sum_{i=1}^{m} f\left(x_{i}\right) p_{i}\right\|<\epsilon / 2 \text { for all } f \in \mathcal{F} .
$$

Define $\Phi_{1}: C(X) \rightarrow B$ by

$$
\Phi_{1}(f)=\psi(f) \oplus \sum_{i=1}^{m} f\left(x_{i}\right) p_{i} \oplus H_{1}(f)
$$

for all $f \in C(X)$ and define $\Phi_{2}: C(X) \rightarrow B$ by

$$
\Phi_{2}(f)=\psi(f) \oplus \sum_{i=1}^{m} f\left(x_{i}\right) p_{i} \oplus H_{2}(f)
$$

for all $f \in C(X)$.

By the choice of $\delta_{4}, \mathcal{G}_{4}$ and $\mathcal{H}_{1}$, and applying [5.2, we obtain that

$$
\left.\operatorname{dist}\left(\overline{\left\langle\Phi_{1}(u)\right\rangle}, \overline{\left\langle\Phi_{2}(u)\right\rangle}\right)<\lambda_{2}^{\prime} / 8 \text { (in } B\right)
$$

for all $u \in \mathcal{U}$ and

$$
\left|T \circ \Phi_{1}(g)-T \circ \Phi_{2}(g)\right|<\lambda_{1}^{\prime} / 4
$$

for all $g \in \mathcal{H}$ and for all $T \in T(B)$.

Combining with (e5.256), we obtain that

$$
\left|t \circ H_{1}(f)-t \circ H_{2}(f)\right| \leq \lambda_{1}^{\prime} / 4+2 \epsilon_{0}<\lambda_{1}^{\prime}
$$

for all $f \in \mathcal{H}$ and for all $t \in T(C)$. Using the de la Harp-Skandalis determinant, combining (e5.260), (e 5.263) and (e5.256), we compute that

$$
\operatorname{dist}\left(\overline{\left\langle H_{1}(u)\right\rangle}, \overline{\left\langle H_{2}(u)\right\rangle}\right)<\lambda_{2}^{\prime} / 4+2 N \epsilon_{0}<\lambda_{2}^{\prime} .
$$

for all $u \in \mathcal{U}$. Then, by (ex.252) and by applying 3.6, there exists a unitary $W_{2} \in C$ such that

$$
\left\|\operatorname{Ad} W_{2} \circ H_{2}(f)-H_{1}(f)\right\|<\epsilon / 2
$$

for all $f \in \mathcal{F}$. Define $W=W_{0}\left(W_{1} \oplus W_{2}\right)$. Then, by (e 5.254), (e 5.262) and (e 5.267), we finally obtain that

$$
\left\|\operatorname{Ad} W \circ L_{2}(f)-L_{1}(f)\right\|<\epsilon
$$

for all $f \in \mathcal{F}$. 
Definition 5.4. Let $X$ be a compact metric space and $P \in M_{r}(C(X))$ be a projection, where $r \geq 1$ is an integer. Put $C=P M_{r}(C(X)) P$. Suppose $\tau \in T(C)$. It is known that there exists a probability measure $\mu_{\tau}$ on $X$ such that

$$
\tau(f)=\int_{X} t_{x}(f(x)) d \mu_{\tau}(x) \text { for all } f \in C
$$

where $t_{x}$ is the normalized trace on $P(x) M_{r} P(x)$ for all $x \in X$ (see 2.17 of [13]).

Suppose that $Y$ is a finite CW complex, $r \geq 1$ is an integer and $P \in M_{r}(C(Y))$ is a projection. Let $X \subset Y$ be a compact subset. Let $\pi: M_{r}(C(Y)) \rightarrow M_{r}(C(X))$ be the quotient map defined by $\pi(f)=\left.f\right|_{X}$ for all $f \in M_{r}(C(Y))$.

Corollary 5.5. Suppose that $Y$ is a finite $C W$ complex, $r \geq 1$ is an integer and $P \in M_{r}(C(Y))$ is a non-zero projection. Define $C=\pi\left(P M_{r}(C(Y)) P\right)$ as defined above. Then Theorem 5.3 holds when $C(X)$ is replaced by $C$ and using the measure defined in 5.4.

Proof. Clearly the corollary holds if $C=M_{r}(C(X))$.

To prove the general case, we may assume that $Y$ is connected. Then there is an integer $d \geq 1$ and a projection $Q \in M_{d}\left(P M_{r}(C(Y)) P\right)$ such that $Q M_{r}\left(P M_{r}(C(Y)) P\right) Q \cong M_{k}(C(Y))$ for some integer $k \geq 1$. Put $C_{1}=Q M_{r}\left(P M_{r}(C(Y)) P\right) Q$. Then there exists a projection $Q_{1} \in M_{k_{1}}\left(C_{1}\right)$ and a unitary $W \in M_{d k_{1}}\left(P M_{r}(C(Y)) P\right)$ such that $W^{*} Q_{1} W=P$. Keep the notation $\pi$ as in 5.4. Note that, for any unital contractive completely positive linear map $L_{i}: C \rightarrow A$, we obtain a unital contractive completely positive linear map $L_{i} \otimes \operatorname{id}_{M_{d}}: M_{r}(C) \rightarrow M_{r}(A)$. Put $\psi_{i, 1}=\left.\left(L_{1} \otimes \operatorname{id}_{M_{r}}\right)\right|_{\pi\left(C_{1}\right)}$ and $\psi_{i, 2}=\left.\left(\psi_{2} \otimes \operatorname{id}_{M_{k_{1}}}\right)\right|_{\pi\left(Q_{1}\right) M_{k_{1}}\left(C_{1}\right) \pi\left(Q_{1}\right)}$. We see that the corollary follows by first considering $\psi_{i, 1}(i=1,2)$ and then $\psi_{i, 2}(i=1,2)$.

Definition 5.6. Let $A$ be a unital $C^{*}$-algebra and let $C$ be another $C^{*}$-algebra. Let $L$ : $C \rightarrow A$ be a positive linear map. Let $\Theta: C_{+} \backslash\{0\} \rightarrow \mathbb{N} \times \mathbb{R}_{+}$be a map. We write $\Theta(c)=$ $(N(\Theta(c)), R(\Theta(c)))$ for $c \in C_{+} \backslash\{0\}$, where $N(\Theta(c)) \in \mathbb{N}$ and $R(\Theta(c)) \in R_{+}$. Suppose that $\mathcal{S} \subset C_{+}$is a subset. We say the map $L$ is $\mathcal{S}$ - $\Theta$-full, if, for each $s \in \mathcal{S}$, there are $x_{1}, x_{2}, \ldots, x_{N(\Theta(s))}$ such that $\left\|x_{j}\right\| \leq R(\Theta(s)), j=1,2, \ldots, N(\Theta(s))$ and

$$
1_{A}=\sum_{j=1}^{N(\Theta(s))} x_{j}^{*} L(s) x_{j} .
$$

The following is known and easy to prove. Only part (1) is actually used in this paper. Both hold for more general unital simple $C^{*}$-algebras. For example, the class of unital separable simple $C^{*}$-algebras which satisfy the strict comparison property for positive elements.

Lemma 5.7. Let $X$ be a compact subset of a finite $C W$ complex $Y$, let $P \in M_{r}(C(Y))$ be a projection, where $r \geq 1$ is an integer, and let $\pi: M_{r}(C(Y)) \rightarrow M_{r}(C(X))$ be defined by $\pi(f)=\left.f\right|_{X}$, Put $C=\pi\left(P M_{r}(C(Y)) P\right)$.

(1) Suppose that $\Theta: C_{+} \backslash\{0\} \rightarrow \mathbb{N} \times\left(\mathbb{R}_{+} \backslash\{0\}\right)$ is a map. Then there exists a non-decreasing map $\Delta:(0,1) \rightarrow(0,1)$ satisfying the following: For any $\eta>0$, there exists a finite subset $\mathcal{S} \subset C_{+} \backslash\{0\}$ such that, if $A$ is a unital separable simple $C^{*}$-algebra with $T R(A) \leq 1$ and if $L: C \rightarrow A$ is a unital $\mathcal{S}-\Theta$-full positive linear map, then

$$
\mu_{\tau \circ L}\left(O_{r}\right) \geq \Delta(r) \text { for all } \tau \in T(A)
$$

for all open balls $O_{r}$ with radius $r \geq \eta$.

(2) Suppose that $\Delta:(0,1) \rightarrow(0,1)$ is a nondecreasing map. Then there exists a map $\Theta: C_{+} \backslash\{0\} \rightarrow \mathbb{N} \times \mathbb{R}_{+} \backslash\{0\}$ satisfying the following: For any finite subset $\mathcal{S} \subset C_{+} \backslash\{0\}$, 
there exists $\eta>0$ such that, if $A$ is a unital separable simple $C^{*}$-algebra with $T R(A) \leq 1$ and $L: C \rightarrow A$ is a unital positive linear map for which

$$
\mu_{\tau \circ L}\left(O_{r}\right) \geq \Delta(r) \text { for all } \tau \in T(A)
$$

for all open balls $O_{r}$ with radius $r \geq \eta$, then $L$ is $\mathcal{S}-\Theta$-full.

Theorem 5.8. Let $C$ be a unital AH-algebra and let $\Theta: C_{+} \backslash\{0\} \rightarrow \mathbb{N} \times \mathbb{R}_{+}$be a map. Let $\epsilon>0, \mathcal{F} \subset C$ be a finite subset. There exists a finite subset $\mathcal{S} \subset A_{+} \backslash\{0\}, \delta>0, \sigma_{1}>0, \sigma_{2}>0$, a finite subset $\mathcal{G} \subset C$, a finite subset $\mathcal{P} \subset \underline{K}(C)$, a finite subset $\mathcal{H} \subset A_{\text {s.a. }}$ and a finite subset $\mathcal{U} \subset U_{c}\left(K_{1}(C)\right)$ satisfying the following: Suppose that $A$ is a unital separable simple $C^{*}$-algebra with $T R(A) \leq 1$ and suppose that $\varphi, \psi: C \rightarrow A$ are two unital $\delta$-G-multiplicative contractive completely positive linear maps such that $\varphi$ and $\psi$ are $\mathcal{S}-\Theta-$ full,

$$
\begin{aligned}
{\left.[\varphi]\right|_{\mathcal{P}} } & =\left.[\psi]\right|_{\mathcal{P}}, \\
|\tau \circ \varphi(g)-\tau \circ \psi(g)| & <\sigma_{1} \text { for all } g \in \mathcal{H}, \\
\operatorname{dist}(\langle\varphi(u)\rangle, \overline{\langle\psi(u)\rangle}) & <\sigma_{2} \text { for all } u \in \mathcal{U} .
\end{aligned}
$$

Then there exists a unitary $w \in A$ such that

$$
\|\operatorname{Ad} w \circ \varphi(f)-\psi(f)\|<\epsilon \text { for all } f \in \mathcal{F} .
$$

Proof. Let $C=\lim _{n \rightarrow \infty}\left(C_{n}, \varphi_{n}\right)$, where $C_{n}=P_{n} M_{r(n)}\left(C\left(Y_{n}\right)\right) P_{n}, X_{n}$ is a finite CW complex, $r(n) \geq 1$ is an integer, $P_{n} \in M_{r(n)}\left(C\left(Y_{n}\right)\right)$ is a projection and $\varphi_{n}: C_{n} \rightarrow C_{n+1}$ is a unital homomorphism. Let $\varphi_{n, \infty}: C_{n} \rightarrow C$ be the unital homomorphism induced by the inductive limit system. Then, for each $n, \varphi_{n, \infty}\left(C_{n}\right) \cong \pi_{n}\left(P_{n}\right) M_{r(n)}\left(C\left(X_{n}\right)\right) \pi_{n}\left(P_{n}\right)$, where $X_{n} \subset Y_{n}$ is a compact subset and $\pi_{n}: M_{r(n)}\left(C\left(Y_{n}\right)\right) \rightarrow M_{r(n)}\left(C\left(X_{n}\right)\right)$ is defined by $\pi_{n}(f)=\left.f\right|_{X_{n}}$. Let $B_{n}=\pi_{n}\left(P_{n}\right) M_{r(n)}\left(C\left(X_{n}\right)\right) \pi_{n}\left(P_{n}\right), n=1,2, \ldots$ Note that $B_{n} \subset B_{n+1}, n=1,2, \ldots$ We may write $C=\overline{\cup_{n=1}^{\infty} B_{n}}$. Let $\epsilon>0$ and $\mathcal{F} \subset C$ be a finite subset. Without loss of generality, we may assume that $\mathcal{F} \subset B_{n}$ for some integer $n \geq 1$. From this it is clear that we can reduce the general case to the case that $C=B_{n}$. Then the result follows from 5.5 and 5.7 ,

Corollary 5.9. Let $C$ be a unital AH-algebra and let $\Theta: C_{+} \backslash\{0\}: \mathbb{N} \times \mathbb{R}_{+}$be a map. For any $\epsilon>0$ and any finite subset $\mathcal{F} \subset C$, there exists $\sigma_{1}>0, \sigma_{2}>0$, a finite subset $\mathcal{S} \subset A_{+} \backslash\{0\}$, a finite subset $\mathcal{P} \subset \underline{K}(C)$, a finite subset $\mathcal{H} \subset A_{\text {s.a. }}$ and a finite subset $\mathcal{U} \subset U_{c}\left(K_{1}(C)\right)$ satisfying the following:

Suppose that $A$ is a unital separable simple $C^{*}$-algebra with $T R(A) \leq 1$ and suppose that $\varphi, \psi: C \rightarrow A$ are two unital monomorphisms which are $\mathcal{S}-\Theta$-full such that

$$
\begin{aligned}
{\left.[\varphi]\right|_{\mathcal{P}} } & =\left.[\psi]\right|_{\mathcal{P}} \\
|\tau \circ \varphi(g)-\tau \circ \psi(g)| & <\sigma_{1} \text { for all } g \in \mathcal{H} \text { and for all } \tau \in T(A), \\
\operatorname{dist}\left(\varphi^{\ddagger}(\bar{u}), \psi^{\ddagger}(u)\right) & <\sigma_{2} \text { for all } u \in \mathcal{U} .
\end{aligned}
$$

Then there exists a unitary $w \in A$ such that

$$
\|\operatorname{Ad} w \circ \varphi(f)-\psi(f)\|<\epsilon \text { for all } f \in \mathcal{F} .
$$

Theorem 5.10. Let $C$ be a unital $A H$-algebra and let $A$ be a unital separable simple $C^{*}$-algebra with $\operatorname{TR}(A) \leq 1$. Suppose that $\varphi, \psi: C \rightarrow A$ are two unital monomorphisms. Then $\varphi$ and $\psi$ are approximately unitarily equivalent if and only if

$$
\begin{aligned}
{[\varphi] } & =[\psi] \text { in } K L(C, A) \\
\varphi_{\sharp} & =\psi_{\sharp} \text { and } \\
\varphi^{\ddagger} & =\psi^{\ddagger} .
\end{aligned}
$$


Theorem 5.11. Let $C$ be a unital $A H$-algebra and let $A$ be a unital separable simple $C^{*}$-algebra with $T R(A) \leq 1$. Suppose that $\varphi, \psi: C \rightarrow A$ are two unital monomorphisms. Then $\varphi$ and $\psi$ are approximately unitarily equivalent if and only if

$$
\begin{aligned}
{[\varphi] } & =[\psi] \text { in } K L(C, A) \\
\varphi_{\sharp} & =\psi_{\sharp} \text { and } \\
\varphi^{\dagger} & =\psi^{\dagger} .
\end{aligned}
$$

Note that $[\varphi]=[\psi], \varphi^{\dagger}=\psi^{\dagger}$ and $\varphi_{\sharp}=\psi_{\sharp}$ imply that $\varphi^{\ddagger}=\psi^{\ddagger}$. Thus Theorem 5.10 follows from 5.9 immediately.

\section{The range}

Definition 6.1. Let $X$ be a compact metric space and let $C=P M_{n}(C(X)) P$, where $P \in$ $M_{n}(C(X))$ is a projection and $P(x)>0$ for all $x \in X$, and let $A$ be a unital separable simple $C^{*}$-algebra with $T(A) \neq \emptyset$. Let $\gamma: T(A) \rightarrow T_{\mathrm{f}}(C)$ be a continuous affine map. For any $\tau \in T(A)$ and any non-empty open set $O \subset X$, define

$$
\mu_{\gamma(\tau)}(O)=\sup \{\gamma(\tau)(f): 0 \leq f<1 \text { and } \operatorname{supp} f \subset O\} .
$$

Since $\gamma(T(A))$ is compact, we conclude that

$$
\inf _{\tau \in T(A)} \mu_{\gamma(\tau)}(O)>0
$$

for every non-empty open subset $O \subset X$.

Fix $a \in(0,1)$. There are finitely many points $x_{1}, x_{2}, . ., x_{m} \in X$ such that $\cup_{n=1}^{m} O\left(x_{i}, a / 2\right) \supset$ $X$. Let $O_{a}$ be an open ball of $X$ with center at a point $x$ and with radius $a$. Then $O_{a} \supset O\left(x_{i}, a / 2\right)$ for some $i$. Define

$$
\Delta_{1}(a)=\min _{\{1 \leq i \leq m\}} \inf _{\tau \in T(A)} \mu_{\gamma(\tau)}\left(O\left(x_{i}, a / 2\right)\right)
$$

for all $a \in(0,1)$. It follows that

$$
\mu_{\tau}\left(O_{a}\right) \geq \Delta_{1}(a) \text { for all } a>0 .
$$

Note that, if $X$ is infinite, $\lim _{a \rightarrow 0} \Delta_{1}(a)=0$.

Lemma 6.2. Let $C$ be as in 6.1 and $A$ be a unital separable simple $C^{*}$-algebra with $T(A) \neq \emptyset$. Suppose that $\gamma: T(A) \rightarrow T_{\mathrm{f}}(C)$ is a continuous affine map. For any $\eta>0,0<\lambda_{1}, \lambda_{2}<1$, there exists a finite subset $\mathcal{H} \subset C_{\text {s.a. }}$ and $\epsilon>0$ satisfying the following: for any unital positive linear map $L: C \rightarrow A$ such that

$$
|\tau \circ L(g)-\gamma(\tau)(g)|<\epsilon \text { for all } g \in \mathcal{H},
$$

then

$$
\mu_{\tau \circ L}\left(O_{r}\right) \geq \lambda_{1} \Delta_{1}(a / 2) / 2\left(1+\lambda_{2}\right) \text { for all } a \geq \eta \text {. }
$$

The proof of this is almost identical to that of 3.4. We omit it.

Lemma 6.3. Let $X$ be a finite $C W$ complex and let $A$ be an infinite dimensional unital simple $C^{*}$-algebra with $T R(A) \leq 1$. Let $C=P M_{r}(C(X)) P(r \geq 1)$, where $P \in M_{r}(C(X))$ is a projection. Suppose that $e \in A$ is a non-zero projection. Then, there exists a non-zero projection $e_{0} \leq e$ and a unital monomorphism $h: C \rightarrow e_{0} A e_{0}$. 
Proof. Without loss of generality, we may assume that $X$ is connected. There are mutually orthogonal and mutually equivalent non-zero projections $e_{1}, e_{2}, \ldots, e_{r} \leq e A e$. Put $e^{\prime}=\sum_{i=1}^{r} e_{i}$. It is well known that there exists a unital monomorphism $h_{0}: C(X) \rightarrow e_{1} A e_{1}$ (see 9.5 of [15]). This extends a monomorphism $h_{1}: M_{r}(C(X)) \rightarrow e^{\prime} A e^{\prime} \cong M_{r}\left(e_{1} A e_{1}\right)$. Let $e_{0}=h_{1}(P)$. Define $h: C \rightarrow e_{0} A e_{0}$ by $h=\left.h_{1}\right|_{C}$.

The following has been used.

Lemma 6.4. Let $A$ be a unital simple separable $C^{*}$-algebra with $T(A) \neq \emptyset$ and let $p \in A$ be a non-zero projection. Then there is an affine homeomorphism $\gamma_{1}: T(A) \rightarrow T(p A p)$ such that, for any extremal point $t \in T(A)$,

$$
\gamma_{1}(t)(a)=t(a) / \tau(p) \text { for all } a \in p A p .
$$

Proof. For each $\tau \in T(A)$, define $\gamma(\tau)(a)=\tau(a) / \tau(p)$ for all $a \in p A p$. It is easy to check that $\gamma: T(A) \rightarrow T(p A p)$ is bijective and bi-continuous. Let $\partial_{e}(A)$ and $\partial_{e}(p A p)$ denote the extremal points of $T(A)$ and $T(p A p)$, respectively. Then $\gamma$ maps $\partial_{e}(T(A))$ onto $\partial_{e}(p A p)$. To see this, let $\tau \in \partial_{e}(T(A))$. Suppose that there are $\tau_{1}, \tau_{2} \in T(p A p)$ such that $\gamma(\tau)=\alpha \tau_{1}+\beta \tau_{2}$, where $0 \leq \alpha, \beta<1$ and $\alpha+\beta=1$. Since $p A p$ is a unital hereditary $C^{*}$-subalgebra, one can extend $\tau_{1}$ and $\tau_{2}$ to two traces on $A$ (see 5.2.7 of [24]). Note also that such extensions are unique, again, because $p A p$ is a hereditary $C^{*}$-subalgebra. Denote $s^{\prime}$ the extension of $\alpha \tau_{1}+\beta \tau_{2}$ this way. Define $s(a)=s^{\prime}(a) / s^{\prime}\left(1_{A}\right)$. Then $s=\tau$. On the other hand, define $t_{i}(a)=\tau_{i}(a) / \tau_{i}\left(1_{A}\right)$ for all $a \in A$ and $i=1,2$. Then

$$
s=s^{\prime} / s^{\prime}\left(1_{A}\right)=\left(\alpha \tau_{1}+\beta \tau_{2}\right) / s^{\prime}\left(1_{A}\right)=\left(\frac{\alpha \tau_{1}\left(1_{A}\right)}{s^{\prime}\left(1_{A}\right)}\right) t_{1}+\left(\frac{\beta \tau_{2}\left(1_{A}\right)}{s^{\prime}\left(1_{A}\right)}\right) t_{2} .
$$

Note $s^{\prime}\left(1_{A}\right)=\alpha \tau_{1}\left(1_{A}\right)+\beta \tau_{2}\left(1_{A}\right)$. Since $s=\tau$ is assumed to be in $\partial_{e}(T(A))$, either

$$
\frac{\alpha \tau_{1}\left(1_{A}\right)}{s^{\prime}\left(1_{A}\right)}=0, \text { or } \frac{\beta \tau_{2}\left(1_{A}\right)}{s^{\prime}\left(1_{A}\right)}=0
$$

which forces either $\alpha=0$ or $\beta=0$. This proves $\gamma$ maps $\partial_{e}(T(A))$ to $\partial_{e}(T(p A p))$. Similar argument shows that $\gamma^{-1}$ maps $\partial_{e}(T(p A p))$ to $\partial_{e}(T(A))$. This implies that $\gamma$ is a homeomorphism from $\partial_{e}(T(A))$ onto $\partial_{e}(T(p A p))$.

For each $\tau \in T(A)$, by the general theory of Choquet, there is a (positive) probability Borel measure $\mu$ on $\partial_{e}(T(A))$ such that

$$
f(\tau)=\int_{\partial_{e}(T(A))} f d \mu \text { for all } f \in \operatorname{Aff}(T(A)) .
$$

Define $\gamma_{1}(\tau)$ by

$$
g\left(\gamma_{1}(\tau)\right)=\int_{\partial_{e}(T(A))} g \circ \gamma_{1} d \mu \text { for all } g \in \operatorname{Aff}(T(p A p) .
$$

It is easy to check that $\gamma_{1}$ is an affine map from $T(A)$ to $T(p A p)$. Since $\left.\gamma_{1}\right|_{\partial_{e}(T(A))}$ is a homeomorphism, $\gamma_{1}$ maps $T(A)$ onto $T(p A p)$ as an affine homeomorphism.

Definition 6.5. Let $C$ and $A$ be two unital $C^{*}$-algebras. Denote by $K K_{e}(C, A)^{++}$the set of those elements $\kappa \in K K(C, A)$ such that

$$
\kappa\left(\left[1_{C}\right]\right)=\left[1_{A}\right] \text { and } \kappa\left(K_{0}(C)_{+} \backslash\{0\}\right) \subset K_{0}(A)_{+} \backslash\{0\} .
$$


Denote by $K L_{e}(C, A)^{++}$the set of those elements $\kappa \in K L(C, A)$ such that $\kappa\left(\left[1_{C}\right]\right)=\left[1_{A}\right]$ and $\kappa\left(K_{0}(C) \backslash\{0\}\right) \subset K_{0}(A)_{+} \backslash\{0\}$.

Now suppose that $T_{\mathrm{f}}(C) \neq \emptyset$ and $A$ is a unital simple $C^{*}$-algebra with $T(A) \neq \emptyset$. Let $\gamma: T(A) \rightarrow T_{\mathrm{f}}(C)$ be a continuous affine map. We say $\kappa$ and $\gamma$ are compatible, if, $\tau \circ \kappa([p])=$ $\gamma(\tau)([p])$ for every projection $p \in M_{\infty}(C)$. Let $\alpha: U\left(M_{\infty}(C)\right) / C U\left(M_{\infty}(C)\right) \rightarrow U(A) / C U(A)$ be a continuous homomorphism. By (e2.5), there is a homomorphism $\alpha_{0}: \operatorname{Aff}(C) / \overline{\rho_{C}\left(K_{0}(C)\right)} \rightarrow$ $\operatorname{Aff}(A) / \overline{\rho_{A}\left(K_{0}(A)\right)}$ induced by $\alpha$ and there is homomorphism $\alpha_{1}: K_{1}(C) \rightarrow K_{1}(A)$ induced by $\alpha$. We say $\alpha$ and $\kappa$ compatible if $\left.\kappa\right|_{K_{1}(C)}=\alpha_{1}$, we say $\kappa, \gamma$ and $\alpha$ are compatible if $\kappa$ and $\gamma$ are compatible, $\kappa$ and $\alpha$ compatible and the homomorphism induced by $\gamma$ is equal to $\alpha_{0}$.

Lemma 6.6. Let $X$ be a finite $C W$ complex, let $n \geq 1$ be an integer, let $C=P M_{n}(C(X)) P$, where $P \in M_{n}(C(X))$ is a projection, and let $A$ be a unital infinite dimensional separable simple $C^{*}$-algebra of tracial rank at most one. Suppose that $\kappa \in K K_{e}(C, A)^{++}$and $\gamma: T(A) \rightarrow T_{\mathrm{f}}(C)$ is a continuous affine map so that $\kappa$ and $\gamma$ are compatible. Let $\sigma>0$ and $\mathcal{H} \subset C_{\text {s.a. }}$ be a finite subset. Then there is a unital monomorphsm $h: C \rightarrow A$ such that

$$
\begin{array}{r}
{[h]=\kappa \text { and }} \\
|\tau \circ h(c)-\gamma(\tau)(c)|<\sigma
\end{array}
$$

for all $c \in \mathcal{H}$ and all $\tau \in T(A)$.

Proof. To simplify the proof, without loss of generality, we may assume that $X$ is connected. We may also assume that $0<\sigma<1 / 2$ and $\mathcal{H}$ is in the unit ball of $C$. There is a unital separable amenable simple $C^{*}$-algebra $B$ with $T R(B)=0$ which satisfies the UCT such that

$$
\left(K_{0}(B), K_{0}(B)_{+},\left[1_{B}\right], K_{1}(B)\right)=\left(K_{0}(A), K_{0}(A)_{+},\left[1_{A}\right], K_{1}(A)\right) .
$$

Let $[\imath] \in K K_{e}(B, A)^{++}$be an invertible element which gives the above identity. Therefore there is $\kappa_{0} \in K K_{e}(C, B)^{++}$such that

$$
\kappa=\kappa_{0} \times[\imath] .
$$

Without loss of generality, we may assume that $\mathcal{H}$ is in the unit ball of $C$.

Let $p \in B$ with $\tau(p)<\sigma / 8$ for all $\tau \in T(B)$. It follows from 6.2 of 20 ] that there is a nonzero projection $p_{0} \leq p$, a finite dimensional $C^{*}$-subalgebra $B_{0} \subset\left(1-p_{0}\right) B\left(1-p_{0}\right)$ with $1_{B_{0}}=1-p_{0}$, a unital homomorphism $h_{1}: C \rightarrow p_{0} B p_{0}$ and a unital homomorphism $h_{2}: C \rightarrow B_{0}$ such that

$$
\left[h_{1}+h_{2}\right]=\kappa_{0} .
$$

Let $e_{0} \in A$ be a projection such that $\left[e_{0}\right]=[\imath]\left(\left[p_{0}\right]\right)$. Put $D=\left(1-e_{0}\right) A\left(1-e_{0}\right)$. Then $D$ is a unital simple $C^{*}$-algebra with $T R(D) \leq 1$. Let $\gamma_{1}^{-1}: T\left(\left(1-e_{0}\right) A\left(1-e_{0}\right)\right) \rightarrow T(A)$ be the affine homeomorphism given by 6.4 such that $\gamma_{1}(\tau)(a)=\tau(a) / \tau\left(1-e_{0}\right)$ for all $a \in\left(1-e_{0}\right) A\left(1-e_{0}\right)$ and $\tau \in \partial_{e}(T(A))$. Note that, for any $\tau \in T(A)$ and $f \in \operatorname{Aff}(T(A))$ with $\|f\| \leq 1$,

$$
\left|f(\tau)-f\left(\gamma_{1}(\tau)\right)\right| \leq \int_{\partial_{e}(T(A))}|f-f \circ \gamma| d \mu<\sup \left\{t\left(e_{0}\right) /\left(1-t\left(e_{0}\right)\right): t \in T(A)\right\}<\sigma / 7,(\mathrm{e} 6.289)
$$

where $f(\tau)=\int_{\partial_{e}(T(A))} f d \mu$ and where $\mu$ is a probability measure on $\partial_{e}(T(A))$. Define $\gamma_{1}^{\prime}$ : $T(D) \rightarrow T_{\mathrm{f}}(C)$ by $\gamma_{1}^{\prime}=\gamma \circ \gamma_{1}^{-1}$. It follows from Lemma 9.5 of [15] that there exists a unital monomorphism $h_{3}: C \rightarrow D$ such that

$$
\begin{aligned}
& {\left[h_{3}\right]=\left[h_{2}\right] \text { in } K K(C, A) .} \\
& \left|t\left(h_{3}(c)\right)-\gamma_{1}^{\prime}(t)(c)\right|<\sigma / 8
\end{aligned}
$$


for all $c \in \mathcal{H}$ and for $t \in T(D)$. It follows from Theorem 5.4 of [20] that there is a unital monomorphism $j: p_{0} B p_{0} \rightarrow e_{0} A e_{0}$ such that $[j]=[\imath]$.

Now define $h: C \rightarrow A$ by $h(c)=j \circ h_{1}(c) \oplus h_{3}(c)$ for all $c \in C$. One computes that

$$
[h]=[\kappa] \text { and }|\tau(h(c))-\gamma(\tau)(c)|<\sigma
$$

for all $c \in \mathcal{H}$ and for all $\tau \in T(A)$.

Lemma 6.7. Let $C$ be as in 6.6 and let $A$ be a unital infinite dimensional separable simple $C^{*}$-algebra with $T R(A) \leq 1$. Suppose that $\kappa \in K K_{e}(C, A)^{++}, \gamma: T(A) \rightarrow T_{\mathrm{f}}(C)$ is a continuous affine map and $\alpha: U\left(M_{\infty}(C)\right) / C U\left(M_{\infty}(C)\right) \rightarrow U(A) / C U(A)$ such that $\kappa, \gamma$ and $\alpha$ are compatible. Then, for any $\sigma_{1}>0,1>\sigma_{2}>0$, any finite subset $\mathcal{H} \subset C_{\text {s.a. }}$ and any finite subset $\mathcal{U} \subset U\left(M_{N}(C)\right.$ ) (for some integer $N \geq 1$ ), there exists a unital monomorphism $h: C \rightarrow A$ such that

$$
\begin{aligned}
{[h]=\kappa, } & |\tau \circ h(c)-\gamma(\tau)(c)|<\sigma_{1} \text { for all } \tau \in T(A) \\
& \text { and } \operatorname{dist}\left(h^{\ddagger}(\bar{u}), \alpha(\bar{u})\right)<\sigma_{2} \text { for all } u \in \mathcal{U} .
\end{aligned}
$$

Proof. To simplify the notation, without loss of generality, we may assume that $X$ is connected. Furthermore, a standard argument shows that, we can further reduce the general case to the case that $C=C(X)$.

We write $K_{1}(C)=G_{1} \oplus \operatorname{Tor}\left(K_{1}(C)\right)$, where $\operatorname{Tor}\left(K_{1}(C)\right)$ is the torsion subgroup of $K_{1}(C)$ and $G_{1}$ is the free part. Fix a point $\xi_{0} \in X$, define

$$
C_{0}=\left\{f \in C: f\left(\xi_{0}\right)=0\right\} .
$$

Then $C_{0} \subset C$ is an ideal of $C$ and $C / C_{0}=\mathbb{C}$. We write

$$
K_{0}(C)=\mathbb{Z}\left[1_{C}\right] \oplus K_{0}\left(C_{0}\right) .
$$

Let $A_{1}$ be a unital separable amenable simple $C^{*}$-algebra with UCT and with $T R\left(A_{1}\right)=$ $T R(A) \leq 1$ such that

$$
\begin{gathered}
\left(K_{0}\left(A_{1}\right), K_{0}\left(A_{1}\right)_{+},\left[1_{A_{1}}\right], T\left(A_{1}\right), \rho_{A_{1}}\right)=\left(K_{0}(A), K_{0}(A)_{+},\left[1_{A}\right], T(A), \rho_{A}\right) \\
\text { and } K_{1}\left(A_{1}\right)=G_{1} \oplus \operatorname{Tor}\left(K_{1}(A)\right) .
\end{gathered}
$$

To simplify notation, we may assume that $\mathcal{U}=\mathcal{U}_{0} \cup \mathcal{U}_{1}$, where $\mathcal{U}_{0} \subset U_{0}\left(M_{N}(C)\right)$ and $\mathcal{U}_{1} \subset$ $U_{c}\left(M_{N}(C)\right)$ are finite subsets and $N \geq 1$ is an integer. For each $u \in \mathcal{U}_{0}$, write $u=\prod_{i=1}^{n(u)} \exp \left(\sqrt{-1} a_{i}(u)\right)$, where $a_{i}(u) \in M_{N}(C)$ is a selfadjoint elements. Write

$$
a_{i}(u)=\left(a_{i}^{(k, j)}(u)\right)_{N \times N}, i=1,2, \ldots, n(u) .
$$

Write

$$
c_{i, k, j}(u)=\frac{a_{i}^{(k, j)}+\left(a_{i}^{(k, j)}\right)^{*}}{2} \text { and } d_{i, k, j}(u)=\frac{a_{i}^{(k, j)}-\left(a_{i}^{(k, j)}\right)^{*}}{2 i}
$$

Put

$$
M=\max \left\{\|c\|,\left\|c_{i, k, j}(u)\right\|,\left\|d_{i, k, j}(u)\right\|: c \in \mathcal{H}, u \in \mathcal{U}_{0}\right\} .
$$

Choose a non-zero projection $e \in A$ such that

$$
\tau(e)<\frac{\sigma_{1}}{8 N^{2}(M+1) \max \left\{n(u): u \in \mathcal{U}_{0}\right\}} \text { for all } \tau \in T(A) .
$$


Let $e_{0} \in A_{1}$ be a projection such that $\left[e_{0}\right]=[e]$ using (e 6.294) and let $A_{2}=\left(1-e_{0}\right) A_{1}\left(1-e_{0}\right)$. In what follows, we use the identification (e6.294). Define $\theta_{1} \in \operatorname{Hom}\left(K_{i}(C), K_{i}\left(A_{2}\right)\right)$ as follows: On $K_{0}(C)$, define $\theta_{1}\left(m\left[1_{C}\right]\right)=m\left[1-e_{0}\right]$ for all $m \in \mathbb{Z},\left.\theta_{1}\right|_{K_{0}\left(C_{0}\right)}=\left.\kappa\right|_{K_{0}\left(C_{0}\right)}$, on $K_{1}(C)$, define

$$
\left.\theta_{1}\right|_{\operatorname{Tor}\left(K_{1}(C)\right)}=\left.\kappa\right|_{\operatorname{Tor}\left(K_{1}(C)\right)},\left.\quad \theta_{1}\right|_{G_{1}}=\left.\mathrm{id}\right|_{G_{1}} .
$$

By the Universal Coefficient Theorem, there exists an element $\theta_{1} \in K L\left(C, A_{2}\right)$ which gives the above homomorphisms. Let $\theta_{2} \in K L\left(A_{1}, A\right)$ which gives the identification (e6.294) and $\left.\theta_{2}\right|_{\operatorname{Tor}\left(K_{1}\left(A_{1}\right)\right)}=\left.\mathrm{id}\right|_{\operatorname{Tor}\left(K_{1}\left(A_{1}\right)\right)}$ and $\left.\theta_{2}\right|_{G_{1}}=\left.\kappa\right|_{G_{1}}$. Let $\beta=\kappa-\theta_{2} \circ \theta_{1}$. We compute that

$$
\begin{array}{r}
\beta\left(\left[1_{C}\right]\right)=[e],\left.\beta\right|_{K_{0}\left(C_{0}\right)}=0, \\
\text { and }\left.\beta\right|_{K_{1}(C)}=0 .
\end{array}
$$

Thus $\beta \in K K_{e}(C, e A e)^{++}$. It follows from 6.6 that there is a unital monomorphism $\varphi_{0}: C \rightarrow e A e$ such that $\left[\varphi_{0}\right]=\beta$.

Choose

$$
\mathcal{H}_{1}=\mathcal{H} \cup\left\{c_{i, k, j}(u), d_{i, k, j}(u): 1 \leq k, j \leq N, 1 \leq i \leq n(u), u \in \mathcal{U}_{0}\right\}
$$

It follows from 6.6 that there exists a unital monomorphism $\varphi_{1}: C \rightarrow A_{2}$ such that

$$
\begin{array}{r}
{\left[\varphi_{1}\right]=\theta_{1} \text { and }} \\
\left|\tau \circ \varphi_{1}(f)-\gamma(\tau)(f)\right|<\frac{\sigma_{1}}{8 N^{2}}
\end{array}
$$

for all $f \in \mathcal{H}_{1}$ for all $\tau \in T(A)$. Note that, for $u \in \mathcal{U}_{0}$,

$$
\Delta(u)=\overline{\sum_{i=1}^{n(u)} \widehat{a_{j}(u)},}
$$

where $\hat{a}(\tau)=\tau(a)$ for all $a \in A_{\text {s.a. }}$. Since $\alpha$ and $\gamma$ are compatible, we then compute that

$$
\operatorname{dist}\left(\varphi_{1}^{\ddagger}(\bar{u}), \alpha(\bar{u})\right)<\sigma_{2} / 8
$$

for all $u \in \mathcal{U}_{0}$. Denote by $U_{c}\left(G_{1}\right)$ the image of $G_{1}$. Define $\chi: U_{c}\left(G_{1}\right) \rightarrow \operatorname{Aff}(T(A)) / \overline{\rho_{A}\left(K_{0}(A)\right)}$ by

$$
\chi=\left.\alpha\right|_{\left.U_{c}\left(G_{1}\right)\right)}-\left.\varphi_{0}^{\ddagger}\right|_{U_{c}\left(G_{1}\right)}-\left.\varphi_{1}^{\ddagger}\right|_{U_{c}\left(G_{1}\right)} .
$$

Note that $U_{c}\left(G_{1}\right) \cong G_{1}$. We identify $U_{c}\left(G_{1}\right)$ with the corresponding part in $U_{c}\left(K_{1}\left(A_{2}\right)\right)$. By defining $\chi$ on $\operatorname{Tor}\left(K_{1}\left(A_{2}\right)\right)$ to be zero, we obtain a homomorphism $\chi: U_{c}\left(K_{1}\left(A_{2}\right)\right) \rightarrow$ $\operatorname{Aff}(T(A)) \overline{\rho_{A}\left(K_{0}(A)\right)}$. It follows from Theorem 8.6 of [20] that there exists a unital homomorphism $h_{1}: A_{2} \rightarrow(1-e) A(1-e)$ such that

$$
\begin{array}{r}
{\left[h_{1}\right]=\theta_{2}, \quad\left(h_{1}\right)_{\sharp}=\operatorname{id}_{T(A)}} \\
\text { and }\left.h_{1}^{\ddagger}\right|_{U_{c}\left(A_{2}\right)}=\chi+\left.\theta_{2}\right|_{K_{1}\left(A_{2}\right)},
\end{array}
$$

where we identify $K_{1}\left(A_{2}\right)$ with $U_{c}\left(A_{2}\right)=U_{c}\left(G_{1}\right) \oplus \operatorname{Tor}\left(K_{1}(A)\right)$ and $U_{c}\left(K_{1}(A)\right)$ with $K_{1}(A)$. We also identify $\operatorname{Aff}\left(T\left(A_{2}\right)\right) \sqrt{\rho_{A}\left(K_{0}\left(A_{2}\right)\right)}$ with $\operatorname{Aff}(T(A)) / \overline{\rho_{A}\left(K_{0}(A)\right)}$. Note that (by (e6.304),

$$
\left.h_{1}^{\ddagger}\right|_{\mathrm{Aff}(T(A)) / \overline{\rho_{A}\left(K_{0}(A)\right)}}=\mathrm{id}_{\mathrm{Aff}(T(A)) / \overline{\rho_{A}\left(K_{0}(A)\right)}} .
$$


Now define

$$
h(f)=\varphi_{0}(f) \oplus h_{1} \circ \varphi_{1}(f) \text { for all } f \in C .
$$

It follows that

$$
\begin{aligned}
{[h] } & =\kappa, \\
|\tau \circ h(f)-\gamma(\tau)(f)| & <\sigma_{1} \text { for all } f \in \mathcal{H} \text { and } \\
\operatorname{dist}\left(h^{\ddagger}(\bar{u}), \alpha(\bar{u})\right) & <\sigma_{2} \text { for all } u \in \mathcal{U} .
\end{aligned}
$$

Lemma 6.8. Let $X$ be a compact subset of a finite $C W$ complex $Y$. Then there exists a sequence of finite $C W$ complex $Y_{n} \supset Y_{n+1}$ each of which is a compact subset of $Y$ and there exists a contractive completely positive linear map $\varphi_{n}: C(X) \rightarrow C\left(Y_{n}\right)$ such that

$$
\begin{gathered}
\pi_{n} \circ \varphi_{n}=\operatorname{id}_{C(X)}, \quad n=1,2, \ldots \text { and } \\
\quad \lim _{n \rightarrow \infty}\left\|\varphi_{n}(f) \varphi_{n}(g)-\varphi_{n}(f g)\right\|=0
\end{gathered}
$$

for all $f, g \in C(X)$, where $\pi_{n}: C\left(Y_{n}\right) \rightarrow C(X)$ is the quotient map.

Proof. Let $d_{n} \searrow 0$ be a decreasing sequence of positive numbers. There are finitely many open balls of $Y$ with center in $X$ and radius $d_{n}$ covers $X$. Let $Z_{n}$ be the union of closure of these balls. Then $Z_{n}$ is a compact subset of $Y$ which is homeomorphic to a finite CW complex. We may assume that $Z_{n} \supset Z_{n+1}$. Then (by, for example, The Effros-Choi Theorem), there exists, for each $n$, a contractive completely positive linear map $\psi_{n}: C(X) \rightarrow C\left(Z_{n}\right)$ such that

$$
\pi_{n} \circ \psi_{n}=\operatorname{id}_{C(X)},
$$

where $\pi_{n}(f)=\left.f\right|_{X}$ for $f \in C\left(Z_{n}\right), n=1,2, \ldots$.

Let $\left\{\mathcal{F}_{m}\right\} \subset C(X)$ be a sequence of increasing finite subsets of the unit ball of $C(X)$ so that its union is dense in the unit ball of $C(X)$. Choose $Y_{1}=Z_{1}$ and $\varphi_{1}=\psi_{1}$. Let $\mathcal{G}_{1}=\mathcal{F}_{1} \cup\{f g$ : $\left.f, g \in \mathcal{F}_{1}\right\}$. Choose $d_{n_{2}}$ such that

$$
\left|\psi_{1}(f)(x)-\psi_{1}(f)\left(x^{\prime}\right)\right|<1 / 4 \text { for all } f \in \mathcal{G}_{1},
$$

provided that $\operatorname{dist}\left(x, x^{\prime}\right)<d_{n_{2}}$ for all $x, x^{\prime} \in Z_{1}$. By (e.312),

$$
\psi_{1}(f g)(x)-\psi_{1}(f)(x) \psi_{1}(g)(x)=0
$$

for all $x \in X$. Now for any $z \in X_{n_{2}}$, there exists $x \in X$ such that $\operatorname{dist}(x, z)<d_{n_{1}}$. Therefore, by (e6.314) and (e6.313),

$$
\begin{aligned}
& \left|\psi_{1}(f g)(z)-\psi_{1}(f)(z) \psi_{1}(g)(z)\right| \leq\left|\psi_{1}(f g)(z)-\psi_{1}(f g)(x)\right| \\
& +\left|\psi_{1}(f g)(x)-\psi_{1}(f)(x) \psi_{1}(g)(x)\right| \\
& +\left|\psi_{1}(f)(x) \psi_{1}(g)(x)-\psi_{1}(f)(z) \psi_{1}(g)(z)\right|<3 / 4
\end{aligned}
$$

for all $f, g \in \mathcal{F}_{1}$. Choose $Y_{2}=Z_{n_{2}}$. Define $h_{1}: C\left(Z_{1}\right) \rightarrow C\left(Z_{n_{2}}\right)$ defined by $h_{1}(f)=\left.f\right|_{Z_{n_{2}}}$ for all $f \in C\left(Z_{1}\right)$. Define $\varphi_{2}: C(X) \rightarrow C\left(Y_{2}\right)$ by defining

$$
\varphi_{2}(f)=h_{1} \circ \psi_{1} .
$$


Thus, by (e6.315),

$$
\left\|\varphi_{2}(f g)-\varphi_{2}(f) \varphi_{2}(g)\right\|<3 / 4
$$

for all $f, g \in \mathcal{F}_{1}$. Note that

$$
\pi_{n_{2}} \circ \varphi_{2}=\operatorname{id}_{C(X)} .
$$

Let $\mathcal{G}_{2}=\mathcal{G}_{1} \cup \mathcal{F}_{2} \cup\left\{f g: f, g \in \mathcal{F}_{2}\right\}$. Choose $d_{n_{3}}$ such that

$$
\left|\varphi_{2}(f)(x)-\varphi_{2}(f)\left(x^{\prime}\right)\right|<1 / 4^{2} \text { for all } f \in \mathcal{G}_{2},
$$

provided that $\operatorname{dist}\left(x, x^{\prime}\right)<d_{n_{3}}$ for all $x, x^{\prime} \in Y_{2}$. By (e.312), for any $x \in X$,

$$
\varphi_{2}(f g)(x)=\varphi_{2}(f)(x) \varphi_{2}(g)(x)
$$

Then, for any $z \in Z_{n_{3}}$, there exists $x \in X$ such that $\operatorname{dist}(x, z)<d_{n_{3}}$. Thus, by (e6.321) and (e6.320),

$$
\left.\mid \varphi_{2}(f g)(z)-\varphi_{2}(f)(z) \varphi_{(} g\right)(z) \mid<3 / 4^{2} \text { for all } z \in Z_{n_{3}} .
$$

Let $h_{2}: C\left(Y_{2}\right) \rightarrow C\left(Z_{n_{3}}\right)$ be defined by $h_{2}(f)=\left.f\right|_{Z_{n_{3}}}$ for all $f \in C\left(Y_{2}\right)$. Put $Y_{3}=Z_{n_{3}}$. Define $\varphi_{3}: C(X) \rightarrow C\left(Y_{n_{3}}\right)$ by $\varphi_{3}(f)=h_{2} \circ \varphi_{2}$. Then

$$
\pi_{n_{3}} \circ \varphi_{3}=\operatorname{id}_{C(X)} .
$$

By (e6.322), we have that

$$
\left\|\varphi_{3}(f g)-\varphi_{3}(f) \varphi_{3}(g)\right\|<3 / 4^{2}
$$

for all $f, g \in \mathcal{F}_{2}$. In this fashion, we obtain a sequence of contractive completely positive linear maps $\varphi_{k}: C(X) \rightarrow C\left(Y_{k}\right)$, where $Y_{k}=Z_{n_{k}}$, such that

$$
\begin{array}{r}
\pi_{n_{k}} \circ \varphi_{k}=\operatorname{id}_{C(X)} \text { and } \\
\left\|\varphi_{k}(f g)-\varphi_{k}(f) \varphi_{k}(g)\right\|<3 / 4^{k-1},
\end{array}
$$

for all $f, g \in \mathcal{F}_{k}, k=1,2, \ldots$ It follows that, for any $f, g \in C(X)$,

$$
\lim _{k \rightarrow \infty}\left\|\varphi_{k}(f g)-\varphi_{k}(f) \varphi_{k}(g)\right\|=0 .
$$

We have the following corollary.

Corollary 6.9. Let $Y$ be a finite $C W$ complex and $P \in M_{r}(C(Y))$ be a non-zero projection for some integer $r \geq 1$. Let $X$ be a compact metric space of $Y$ and let $C=\pi\left(P M_{r}(C(Y)) P\right)$, where $\pi: M_{r}(C(Y)) \rightarrow M_{r}(C(X))$ is the quotient map defined by $\pi(f)=\left.f\right|_{X}$. Then there exists a sequence of finite $C W$ complex $Y \supset Y_{n} \supset Y_{n+1}$ each of which is a compact subset of $Y$ and there exists a contractive completely positive linear map $\varphi_{n}: C \rightarrow P_{n}\left(C\left(Y_{n}\right)\right) P_{n}$ such that

$$
\begin{aligned}
& \pi_{n} \circ \varphi_{n}=\operatorname{id}_{C}, \quad n=1,2, \ldots \text { and } \\
& \lim _{n \rightarrow \infty}\left\|\varphi_{n}(f) \varphi_{n}(g)-\varphi_{n}(f g)\right\|=0
\end{aligned}
$$

for all $f, g \in C(X)$, where $P_{n}=\left.P\right|_{Y_{n}}$ and $\pi_{n}: C\left(Y_{n}\right) \rightarrow C(X)$ is the quotient map defined by $\pi_{n}(f)=\left.f\right|_{X}$ for all $f \in C\left(Y_{n}\right)$. 
Lemma 6.10. Let $Y$ be a finite $C W$ complex and $P \in M_{r}(C(Y))$ be a non-zero projection for some integer $r \geq 1$. Let $X$ be a compact metric space of $Y$ and let $C=\pi\left(P M_{r}(C(Y)) P\right)$, where $\pi: M_{r}(C(Y)) \rightarrow M_{r}(C(X))$ is the quotient map defined by $\pi(f)=\left.f\right|_{X}$. Suppose that $A$ is a unital infinite dimensional separable simple $C^{*}$-algebra with $T R(A) \leq 1$. For any $\kappa \in$ $K L_{e}(C, A)^{++}$, any affine continuous map $\gamma: T(A) \rightarrow T_{\mathrm{f}}(C)$ and any continuous homomorphism $\alpha: U\left(M_{\infty}(C)\right) / C U\left(M_{\infty}(C)\right) \rightarrow U(A) / C U(A)$ such that $\kappa, \gamma$ and $\alpha$ are compatible, then there is a unital monomorphism $h: C \rightarrow A$ such that

$$
[h]=\kappa, h_{\sharp}=\gamma \text { and } h^{\ddagger}=\alpha \text {. }
$$

Proof. Let $Y_{n}, P_{n}, \pi_{n}$ and $\varphi_{n}$ be as given by 6.9. Let $B_{n}=P_{n} M_{r}\left(C\left(Y_{n}\right)\right) P_{n}$. Let $\left\{q_{n}^{\prime}\right\}$ be a sequence of non-zero projections such that $\tau\left(q_{n}^{\prime}\right)<1 / n$ for all $\tau \in T(A), n=1,2, \ldots$. Since $q_{n}^{\prime} A q_{n}^{\prime}$ is a unital infinite dimensional simple $C^{*}$-algebra of tracial rank at most one, by 6.3 , there exists a non-zero projection $q_{n} \leq q_{n}^{\prime}$ and a unital monomorphism $\varphi_{0, n}: B_{n} \rightarrow q_{n} A q_{n}$, $n=1,2, \ldots$

Define $\gamma_{n}: T(A) \rightarrow T_{\mathrm{f}}\left(B_{n}\right)$ by

$$
\gamma_{1}(\tau)(b)=\tau\left(1-q_{n}\right) \gamma(\tau)\left(\pi_{n}(b)\right)+\tau \circ \varphi_{0, n}(b)
$$

for all $b \in B_{n}$ and for all $\tau \in T(A)$.

Define $s_{n}: B_{n} \rightarrow B_{n+1}$ by $s_{n}(f)=\left.f\right|_{Y_{n}}$ for all $f \in B_{n}, n=1,2, \ldots$

Let $\kappa_{n}=\kappa \circ\left[\pi_{n}\right]$ be in $\operatorname{Hom}_{\Lambda}\left(\underline{K}\left(B_{n}\right), \underline{K}(A)\right)$ and let $\alpha_{n}: U\left(M_{\infty}\left(B_{n}\right)\right) / C U\left(M_{\infty}(C)\right) \rightarrow$ $U(A) / C U(A)$ defined by $\alpha_{n}=\alpha \circ \pi_{n}^{\ddagger}$.

Note that $K_{i}\left(B_{n}\right)$ is finitely generated $(i=0,1)$. Let $\epsilon_{n}>0, \mathcal{F}_{n} \subset B_{n}$ be a finite subset and $Q_{n} \subset \underline{K}\left(B_{n}\right)$ be a finite subset such that $\left(\epsilon_{n}, \mathcal{F}_{n}, \mathcal{P}_{n}\right)$ is a $\underline{K}$-triple and $\left(\epsilon_{n}, \mathcal{F}_{n}\right)$ is $K K$-pair for $B_{n}, n=1,2, \ldots$. Put $\mathcal{Q}_{n}=\left[\pi_{n}\right]\left(\mathcal{P}_{n}\right), n=1,2, \ldots$. We may assume that $\left[s_{n}\right]\left(\mathcal{P}_{n}\right) \subset \mathcal{P}_{n+1}$ and $\cup_{n=1}^{\infty} \mathcal{Q}_{n}=\underline{K}(C)$. Let $\mathcal{G}_{n} \subset C$ be a finite subset and let $\delta_{n}>0$ be such that $\left(\delta_{n}, \mathcal{G}_{n}, \mathcal{Q}_{n}\right)$ is a $\underline{K}$-triple.

Choose, for each $n$, a finite subset $\mathcal{F}_{n} \subset B_{n}$ such that $s_{n}\left(\mathcal{F}_{n}\right) \subset \mathcal{F}_{n+1}$ and $\cup_{n=1}^{\infty} \pi_{n}\left(\mathcal{F}_{n}\right)$ is dense in $C$. Choose, for each $n$, a finite subset $\mathcal{H}_{n} \subset\left(B_{n}\right)_{s . a}$ such that $s_{n}\left(\mathcal{H}_{n}\right) \subset \mathcal{H}_{n+1}$ and $\cup_{n=1}^{\infty} \pi_{n}\left(\mathcal{H}_{n}\right)$ is dense in $C_{\text {s.a. }}$. Choose, for each $n$, a finite subset $\mathcal{U}_{n} \subset U\left(M_{N(n)}\left(B_{n}\right)\right.$ ) (for some integer $N(n))$ such that $s_{n}\left(\mathcal{U}_{n}\right) \subset \mathcal{U}_{n+1}$ and $\cup_{n=1}^{\infty} \pi_{n}\left(\mathcal{U}_{n}\right)$ is dense in $U\left(M_{\infty}(C)\right)$.

It follows from 6.7 there is, for each $n$, a unital monomorphism $h_{n}: B_{n} \rightarrow A$ such that

$$
\left[h_{n}\right]=\kappa_{n}
$$

$\left|\tau \circ h_{n}(f)-\gamma_{n}(\tau)(f)\right|<1 / 2^{n}$ for all $f \in \mathcal{H}_{n}$ and for all $\tau \in T(A)$

and $\operatorname{dist}\left(h_{n}^{\ddagger}(\bar{u}), \alpha_{n}(\bar{u})\right)<1 / 2^{n}$ for all $u \in \mathcal{U}_{n}$,

$n=1,2, \ldots$. Define $L_{n}=h_{n} \circ \varphi_{n}$. Note that

$$
\begin{aligned}
\pi_{n} \circ \varphi_{n} & =\operatorname{id}_{C} \text { and } \\
\lim _{n \rightarrow \infty}\left\|\varphi_{n}(f g)-\varphi_{n}(f) \varphi_{n}(g)\right\| & =0 \text { for all } f, g \in C .
\end{aligned}
$$

Thus, without loss of generality, we may assume that $\varphi_{n}$ is $\left(\delta_{n-1}, \mathcal{G}_{n-1}\right)$-multiplicative. It then follows that from (e6.335) and (e6.332) that

$$
\left.\left[h_{n} \circ \varphi_{n}\right]\right|_{\mathcal{Q}_{n}}=\left.\left(\kappa_{n}\right)\right|_{\mathcal{Q}_{n}}, \quad n=1,2, \ldots
$$

By (e 6.337), (e-6.333), (e6.334), combining 6.2 and applying 5.5, we obtain a subsequence $h_{n_{k}} \circ \varphi_{n_{k}}: C \rightarrow A$ and a sequence of unitaries $\left\{u_{k}\right\} \subset A$ such that

$$
\left\|\operatorname{Ad} u_{k} \circ h_{n_{k+1}} \circ \varphi_{n_{k+1}}(f)-\operatorname{Ad} u_{k-1} \circ h_{n_{k}} \circ \varphi_{n_{k}}(f)\right\|<1 / 2^{k+1}
$$


for all $f \in \mathcal{F}_{k}$. It follows that $\left\{\operatorname{Ad} u_{k-1} \circ h_{n_{k}} \circ \varphi_{n_{k}}(f)\right\}$ is Cauchy for all $f \in C$. Define $h: C \rightarrow A$ by

$$
h(f)=\lim _{k \rightarrow \infty} \operatorname{Ad} u_{k-1} \circ h_{n_{k}} \circ \varphi_{n_{k}}(f) \text { for all } f \in C .
$$

It is ready to check that $h$ satisfy all requirements of the lemma.

Theorem 6.11. Let $C$ be a unital $A H$-algebra and let $A$ be a unital infinite dimensional separable simple $C^{*}$-algebra with $T R(A) \leq 1$. For any $\kappa \in K L_{e}(C, A)^{++}$, any affine continuous map $\gamma: T(A) \rightarrow T_{\mathrm{f}}(C)$ and any continuous homomorphism $\alpha: U\left(M_{\infty}(C)\right) / C U\left(M_{\infty}(C)\right) \rightarrow$ $U(A) / C U(A)$ such that $\kappa, \gamma$ and $\alpha$ are compatible, then there is a unital monomorphism $h$ : $C \rightarrow A$ such that

$$
[h]=\kappa, \quad h_{\sharp}=\gamma \text { and } h^{\ddagger}=\alpha .
$$

Proof. Write $C=\lim _{n \rightarrow \infty}\left(B_{n}, \psi_{n}\right)$, where $B_{n}=P_{n} M_{r(n)}\left(C\left(Y_{n}\right)\right) P_{n}, Y_{n}$ is a finite CW complex, $P_{n} \in M_{r(n)}\left(C\left(Y_{n}\right)\right)$ is a projection and $\psi_{n}: B_{n} \rightarrow B_{n+1}$ is a unital homomorphism. Denote by $\psi_{n, \infty}: B_{n} \rightarrow C$ the unital homomorphism induced by the inductive limit system. Then $\varphi_{n, \infty}\left(B_{n}\right) \cong Q_{n} M_{r(n)}\left(C\left(X_{n}\right)\right) Q_{n}$, where $X_{n} \subset Y_{n}$ is a compact subset, $Q_{n}=\pi_{n}\left(P_{n}\right)$ and where $\pi_{n}: M_{r(n)}\left(C\left(Y_{n}\right)\right) \rightarrow M_{r(n)}\left(C\left(X_{n}\right)\right)$ is the quotient map defined by $\pi_{n}(f)=\left.f\right|_{X_{n}}$. Put $C_{n}=\varphi_{n, \infty}\left(B_{n}\right)$. We will identify $C_{n}$ with $Q_{n} M_{r(n)}\left(C\left(X_{n}\right)\right) Q_{n}$ and write $C=\overline{\cup_{n=1}^{\infty} C_{n}}$, where $C_{n}=Q_{n} M_{r(n)}\left(C\left(X_{n}\right) Q_{n}\right.$,

Denote by $\imath_{n}: C_{n} \rightarrow C_{n+1}$ and $\imath_{n, \infty}: C_{n} \rightarrow C$ be the embedding, respectively. Let $\kappa_{n}=$ $\kappa \circ\left[\imath_{n, \infty}\right]$ be in $\operatorname{Hom}_{\Lambda}\left(\underline{K}\left(C_{n}\right), \underline{K}(A)\right)$ and let $\alpha_{n}: U\left(M_{\infty}\left(C_{n}\right)\right) / C U\left(M_{\infty}(C)\right) \rightarrow U(A) / C U(A)$ be defined by $\alpha_{n}=\alpha \circ \imath_{n, \infty}^{\ddagger}$. Let $\left(\imath_{n, \infty}\right)_{\sharp}: T_{\mathrm{f}}(C) \rightarrow T_{\mathrm{f}}\left(C_{n}\right)$ be induced by $\imath_{n, \infty}$ and define $\gamma_{n}: T(A) \rightarrow T_{f}\left(C_{n}\right)$ by $\left(\imath_{n, \infty}\right)_{\sharp} \circ \gamma$. Put $\mathcal{Q}_{n}=\left[\imath_{n, \infty}\right]\left(\mathcal{P}_{n}\right), n=1,2, \ldots$. We may assume that $\cup_{n=1}^{\infty} \mathcal{Q}_{n}=\underline{K}(C)$. Choose, for each $n$, a finite subset $\mathcal{F}_{n} \subset C_{n}$ such that $\mathcal{F}_{n} \subset \mathcal{F}_{n+1}$ and $\cup_{n=1}^{\infty} \mathcal{F}_{n}$ is dense in $C$. It follows from 6.10 there is, for each $n$, a unital monomorphism $\varphi_{n}: C_{n} \rightarrow A$ such that

$$
\left[\varphi_{n}\right]=\kappa_{n}, \quad\left(\varphi_{n}\right)_{\sharp}=\gamma_{n} \text { and } \varphi_{n}^{\ddagger}=\alpha_{n}
$$

$n=1,2, \ldots$ By applying 5.10 , for each $n$, there exists a unitary $u_{n} \in A$ (with $u_{0}=1$ ) such that

$$
\left\|\operatorname{Ad} u_{n} \circ \varphi_{n+1} \circ \imath_{n}(f)-\operatorname{Ad} u_{n-1} \circ \varphi_{n}(f)\right\|<1 / 2^{n} \text { for all } f \in \mathcal{F}_{n},
$$

$n=1,2, \ldots$ We obtain a unital monomorphism $h: C \rightarrow A$ such that

$$
h(f)=\lim _{n \rightarrow \infty} \operatorname{Ad} u_{n} \circ \varphi_{n+1} \circ \imath_{n}(f) \text { for all } f \in C .
$$

One checks that $h$ meets all requirements of the theorem.

Corollary 6.12. Let $C$ be a unital $A H$-algebra and let $A$ be a unital infinite dimensional separable simple $C^{*}$-algebra with $T R(A) \leq 1$. For any $\kappa \in K L_{e}(C, A)^{++}$, any affine continuous map $\gamma: T(A) \rightarrow T_{\mathrm{f}}(C)$ and any continuous homomorphism $\alpha: K_{1}(C) \rightarrow \operatorname{Aff}(T(A)) / \overline{K_{0}(A)}$ such that $\kappa, \gamma$ are compatible, then there is a unital homomorphism $h: C \rightarrow A$ such that

$$
[h]=\kappa, \quad h_{\sharp}=\gamma \text { and } h^{\dagger}=\alpha \text {. }
$$




\section{References}

[1] J. Cuntz and G. K. Pedersen, Equivalence and traces on $C^{*}$-algebras, J. Funct. Anal. 33 (1979), 135-164.

[2] M. Dadarlat, Approximately unitarily equivalent morphisms and inductive limit $C^{*}$ algebras, K-Theory 9 (1995), 117-137.

[3] M. Dadarlat and S. Eilers, On the classification of nuclear $C^{*}$-algebras, Proc. London Math. Soc. 85 (2002), 168-210.

[4] G. A. Elliott, G. Gong and L. Li, On the classification of simple inductive limit $C^{*}$ algebras. II, The isomorphism theorem, Invent. Math. 168 (2007), 249-320

[5] G. Gong, On the classification of simple inductive limit $C^{*}$-algebras. I, The reduction theorem, Doc. Math. 7 (2002), 255-461.

[6] G. Gong and H. Lin, Classification of homomorphisms from $C(X)$ to simple $C^{*}$-algebras of real rank zero, Acta Math. Sin. (Engl. Ser.) 16 (2000), 181-206

[7] G. Gong and H. Lin, Almost multiplicative morphisms and K-theory, Internat. J. Math. 11 (2000), 983-1000.

[8] H. Lin, Approximation by normal elements with finite spectra in $C^{*}$-algebras of real rank zero, Pacific J. Math. 173 (1996), 443-489.

[9] H. Lin, Homomorphisms from $C(X)$ into $C^{*}$-algebras, Canad. J. Math. 49 (1997), 9631009.

[10] H. Lin, When almost multiplicative morphisms are close to homomorphisms, Trans. Amer. Math. Soc. 351 (1999), 5027-5049.

[11] H. Lin, An introduction to the classification of amenable $C^{*}$-algebras, World Scientific Publishing Co., Inc., River Edge, NJ, 2001. xii+320 pp. ISBN: 981-02-4680-3.

[12] H. Lin, The tracial topological rank of $C^{*}$-algebras, Proc. London Math. Soc. 83 (2001), 199-234.

[13] H. Lin, Traces and simple $C^{*}$-algebras with tracial topological rank zero, J. Reine Angew. Math. 568 (2004), 99-137.

[14] H. Lin, Classification of homomorphisms and dynamical systems, Trans. Amer. Math. Soc. 359 (2007), 859-895.

[15] H. Lin, Simple nuclear $C^{*}$-algebras of tracial topological rank one, J. Funct. Anal. 251 (2007), 601-679.

[16] H. Lin, Asymptotically unitary equivalence and asymptotically inner automorphisms, Amer. J. Math. 131 (2009), 1589-1677,

[17] H. Lin, Approximate homotopy of homomorphisms from $C(X)$ into a simple $C^{*}$-algebra, Mem. Amer. Math. Soc. 205 (2010), no. 963, vi+131 pp.

[18] H. Lin, Homotopy of unitaries in simple $C^{*}$-algebras of tracial rank one, J. Funct. Anal. 258 (2010), 1822-1882. 
[19] H. Lin, Unitaries in a simple $C^{*}$-algebra of tracial rank one, Inter. J. Math., 21 (2010), 1267-1281.

[20] H. Lin, Asymptotically unitary equivalence and classification of simple amenable $C^{*}$ algebras, Inven. Math. 183, (2011), 385-450.

[21] H. Lin, Approximate unitary equivalence in simple $C^{*}$-algebras of tracial rank one, Trans. Amer. Math. Soc. 364 (2012), 2021-2086.

[22] H. Lin, On local AH-algebras, Memoirs Amer. Math. Soc., (2015), vol. 235, no.1107, $1-109$.

[23] S. Mardesic. On covering dimension and inverse limits of compact spaces, Illinois J. Math., 4 (1960), 278-291.

[24] G. K. Pedersen, $C^{*}$-algebras and their Automorphism Groups, Academic Press, London/New York/ San Francisco, 1979, ISBN 0-12-549450-5.

[25] N. C. Phillips, Reduction of exponential rank in direct limits of $C^{*}$-algebras, Canad. J. Math. 46 (1994), 818-853.

[26] J. Rosenberg and C. Schochet, The Knneth theorem and the universal coefficient theorem for Kasparov's generalized K-functor, Duke Math. J. 55 (1987), 431-474.

[27] K. Thomsen, Traces, unitary characters and crossed products by Z, Publ. Res. Inst. Math. Sci. 31 (1995), 1011-1029. 